\title{
WestVirginiaUniversity
}

THE RESEARCH REPOSITORY @ WVU

Graduate Theses, Dissertations, and Problem Reports

2012

\section{Calculating the Surface Seismic Signal from a Trapped Miner}

Adeniyi A. Adebisi

West Virginia University

Follow this and additional works at: https://researchrepository.wvu.edu/etd

\section{Recommended Citation}

Adebisi, Adeniyi A., "Calculating the Surface Seismic Signal from a Trapped Miner" (2012). Graduate

Theses, Dissertations, and Problem Reports. 2282.

https://researchrepository.wvu.edu/etd/2282

This Thesis is protected by copyright and/or related rights. It has been brought to you by the The Research Repository @ WVU with permission from the rights-holder(s). You are free to use this Thesis in any way that is permitted by the copyright and related rights legislation that applies to your use. For other uses you must obtain permission from the rights-holder(s) directly, unless additional rights are indicated by a Creative Commons license in the record and/ or on the work itself. This Thesis has been accepted for inclusion in WVU Graduate Theses, Dissertations, and Problem Reports collection by an authorized administrator of The Research Repository @ WVU. For more information, please contact researchrepository@mail.wvu.edu. 


\title{
Calculating the Surface Seismic Signal from a Trapped Miner
}

\author{
Adeniyi A. Adebisi \\ Thesis Submitted to the \\ College of Engineering and Mineral Resources \\ at West Virginia University \\ in partial fulfillment of the requirements \\ for the degree of \\ Master of Science \\ in \\ Mining Engineering
}

Keith A. Heasley, Ph.D., P.E.

Syd S. Peng, Ph.D.

Yi Luo, Ph.D., P.E.

Mining Engineering

Morgantown, West Virginia

2012

Keywords:

Pounding Factor, Proportionality Constant, Hammer Momentum, Roof Momentum, Signal force time function, Displacement time function and Peak Particle Velocity 


\section{ABSTRACT \\ Calculating the Surface Seismic Signal from a Trapped Miner}

\section{Adeniyi A. Adebisi}

The recent Sago mine disaster has highlighted the need to fundamentally analyze the practical detection limitations of a mine rescue seismic system (Heasley, 2006). In various field tests, it was discovered that the surface peak particle velocity resulting from the trapped miner who pounds on the mine roof becomes undetectable past a certain distance from the surface geophones. In order to provide the mine rescuer the understanding of the exact extent of this distance; and the foreknowledge about the excepted magnitude of the surface peak particle movement in terms of: depth, geology and offset angle, this study was performed.

To achieve this goal, the following three major components were investigated: 1) the roof displacement resulting from the momentum of the impact of the signaling device when it hits the roof, 2) the pounding factor that relates the pounding instruments (sledgehammer or crib block) to the frequency and amplitude of the seismic signal, and 3) the attenuation of the displacement pulse as it moves through the overburden to the surface. To obtain these values, a parallel study of theoretical and experimental work was done (because of the uncertainties still associated with the mechanics of the roof momentum). In the theoretical analysis, a numerical model of the expected seismic attenuation of a given mine site was developed using an EXCEL spreadsheet. This model uses mine specific geology with the appropriate seismic parameters and vertical and horizontal offset distances to determine the expected attenuation. For the estimate of the pounding factor, the numerical modeling of the 4-West Mine where a field test took place was done to determine the seismic attenuation of the mine; and then the peak particle movements that were recorded during the seismic field experiment were back-analyzed to estimate the pounding factor. 
With this simplified numerical analysis of the seismic attenuation and the representative' values for the pounding device pounding factor; the mine rescuer will be able to determine: 1) the expected magnitude of the surface peak particle movement, 2) the limiting distance where the signal would likely become undetectable, and 3) the chance of detecting a miner's signal with respect to the background noise level, and the appropriate steps to follow (either to implements appropriate procedures in order to reduce the noise level or to redirect the rescue efforts). It is believed that the information itemized above will be very useful to facilitate the optimization of the future mine rescue seismic deployment plan. 


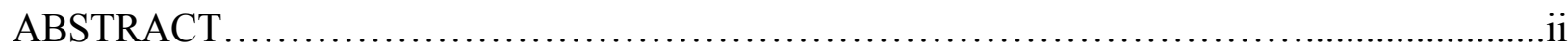

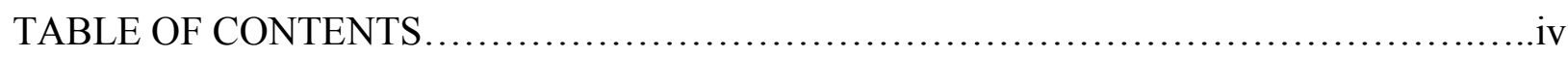

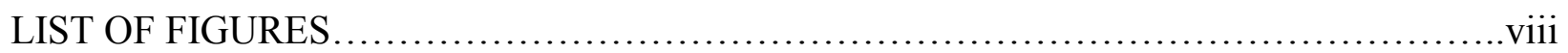

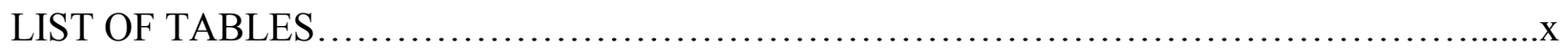

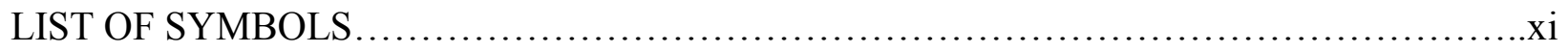

ACKNOWLEDGMENTS ............................................................

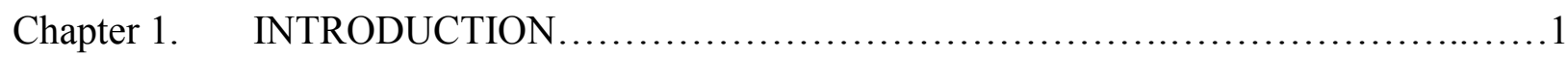

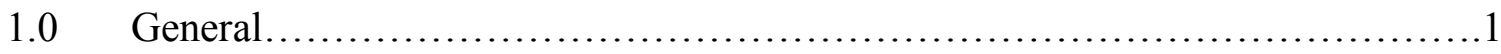

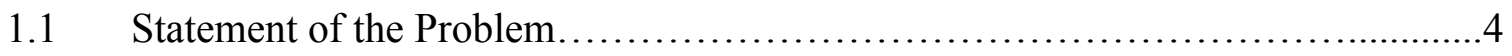

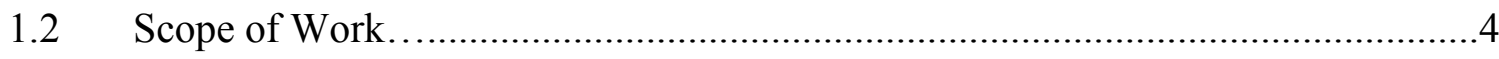

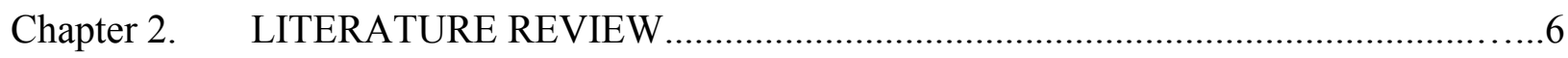

2.0 The Physics of Seismic Waves.........................................................................

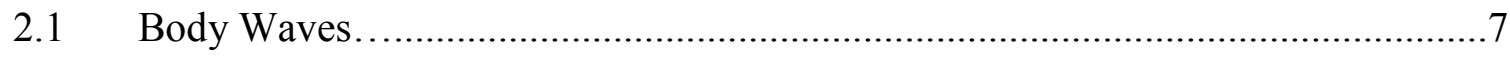

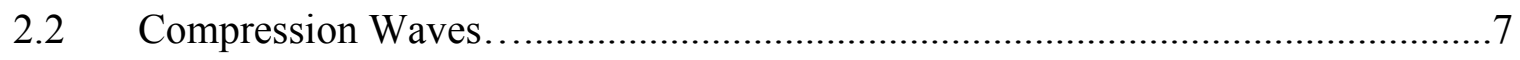

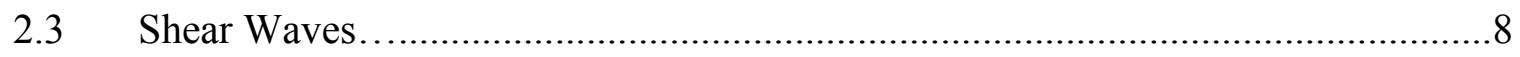

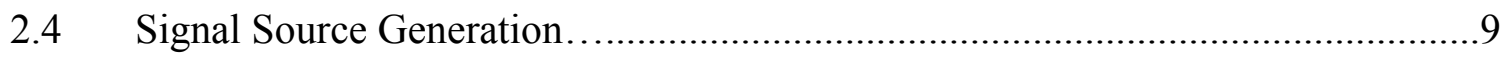

2.5 Numerical Representation of Signal Source.....................................................10

$2.6 \quad$ Particle Velocity ...............................................................

2.7 Geophone 
2.8 Coal Mine Event Location Studies....................................................................13

Chapter 3. CALCULATING THE SURFACE SIGNAL STRENGTH...................................20

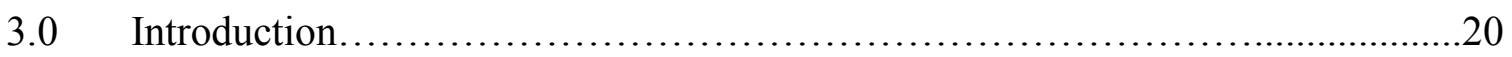

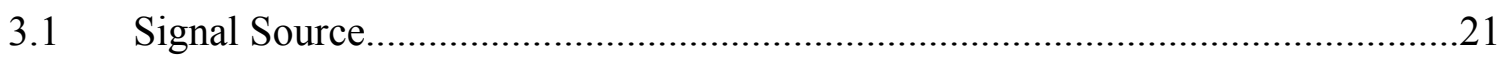

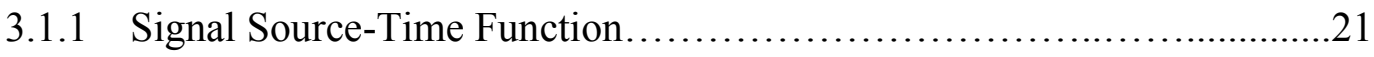

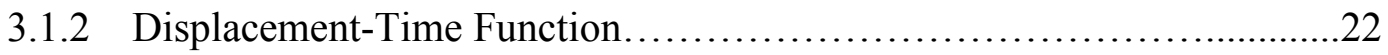

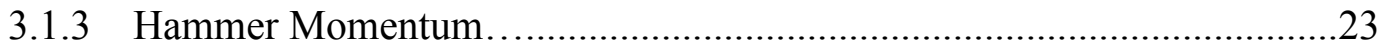

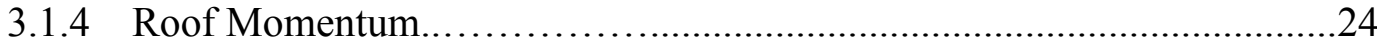

3.1.5 Proportionality Constant ..................................................................25

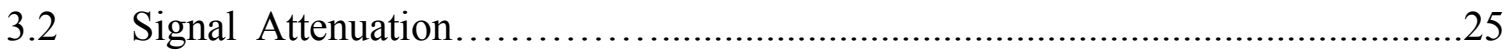

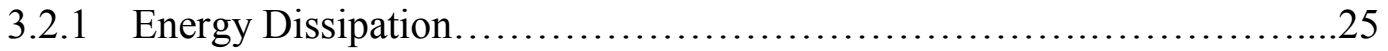

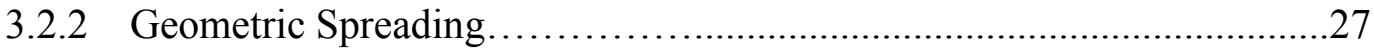

3.2.3 Inter-bed Reflection/Transmission...................................30

3.2.4 Summary of Signal Loss Mechanisms.......................................................33

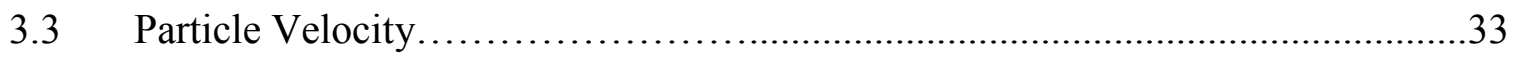

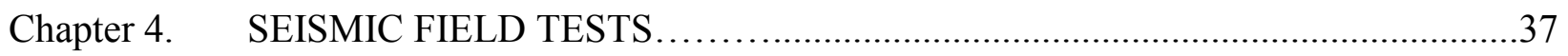

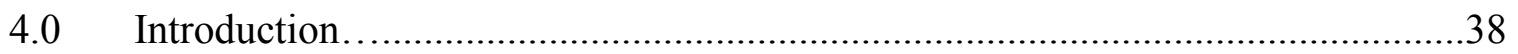

4.1 Field Study at 4-West Mine of Dana Mining ................................................38

4.1.1 Geological Layers of 4-West Mine of Dana Mining ......................39

4.2 Seismic System Installation at the 4-West Mine of Dana Mining ........................41 
4.3 Seismic Equipment used at 4-West Dana Mining....................................42

4.3.1 Terrascience Microseismic Acquisition................................................43

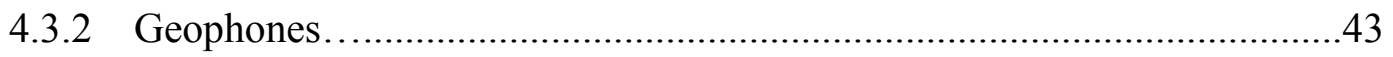

4.3.3 Signaling Devices...................................................................4 44

4.4 Test Protocol for the 4-West Mine of Dana Mining Field Study.......................44

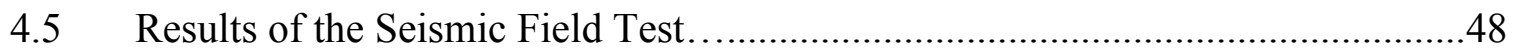

4.6 Second Field Test at Federal \#2 Mine of Peabody Energy........................51

4.6.1 Geophone Locations of the Field Test.....................................................53

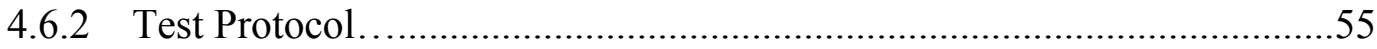

4.6.3 Seismic Field Test Results..............................................................57

Chapter 5. ESTIMATING THE POUNDING FACTOR ..........................58

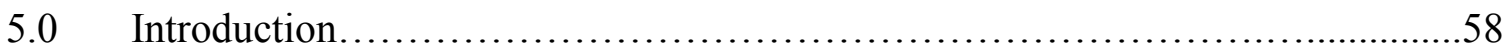

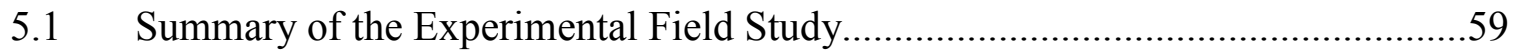

5.2 Theoretical Analysis of Field Study and Pounding Factor Computation..........60

5.2.1 Derivation of Pounding Factor Equation...........................................60

5.2.2 Attenuation Modeling of 4 West Mine in an EXCEL Spreadsheet..........61

5.2 .3 Impedance.................................................63

5.2.4 Angle of Refraction and Transmission..........................63

5.2.5 Offset and Radial Distance..............................................64

5.2.6 Signal Travel Time...............................................66

5.2.7 Angle of Incidence at the Pounding Surface..........................66

5.2.8 Results of the Attenuation model of the 4-West Mine of Dana Mining...67

5.3 Pounding Factor for the crib block...................................68 
5.4 Pounding Factor for sledgehammer.........................................................69

$5.5 \quad$ Validating the Results of the Pounding Factors............................ 70

5.6 Conclusion on the Validation Results...................................... 71

5.7 Calculating the Surface Peak Particle Velocity Using the EXCEL Program.......72

$5.8 \quad$ Validation of the PPV EXCEL Program........................................................ 74

Chapter 6 CONCLUSIONS AND RECOMMENDATIONS .......................................77

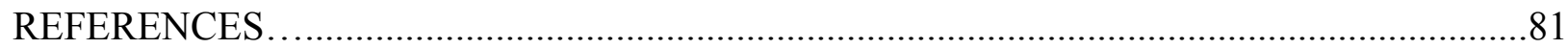




\section{LIST OF FIGURES}

Figure $1.0 \quad$ Schematic of seismic signal and signal propagation............................

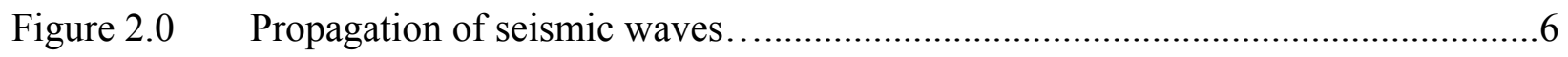

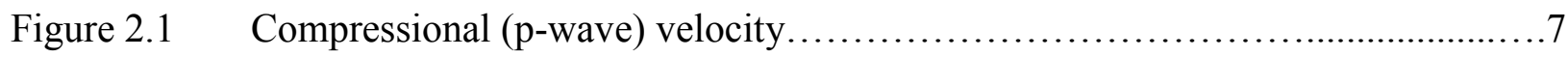

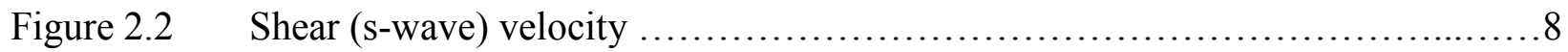

Figure $2.3 \quad$ Pounding with a Sledgehammer..............................................

Figure 2.4 Waveform of a typical signal source generated by an impulsive force...............11

Figure $2.5 \quad$ Geophone components.................................................. 13

Figure $2.6 \quad$ Three component geophone.......................................... 14

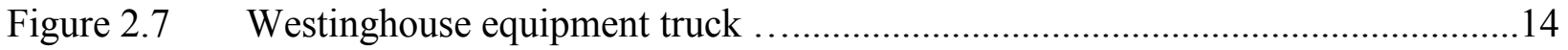

Figure 2.8 Compressional velocity of various sedimentary materials...................... 16

Figure 3.0 Block diagram showing signal generation, transmission and detection on the

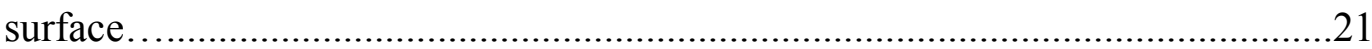

Figure $3.1 \quad$ A typical miner's signal source................................................................22

Figure 3.2 A typical wave form pattern for a miner's signal.......................................24

Figure 3.3 Attenuation from a point source due to spherical geometric spreading..............29

Figure 3.4 Signal reflection and transmission at the interface........................................32

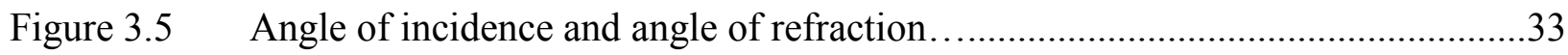

Figure 4.0 Location map of the 4-West Mine of Dana Mining seismic test site...................39

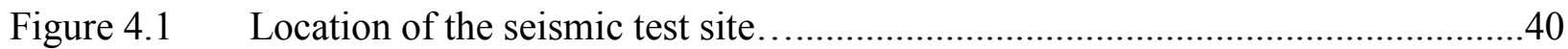

Figure 4.2 Location of the surface geophones and underground pounding sites................42

Figure 4.3 Terrascience's micro seismic acquisition equipment...................................44

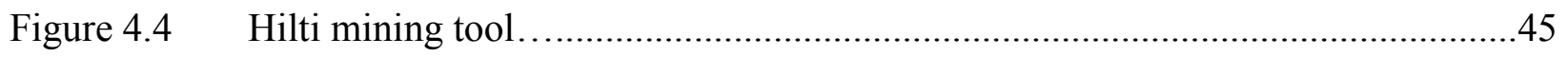


Figure 4.5 Comparaison between seismic signal generated with a crib block and

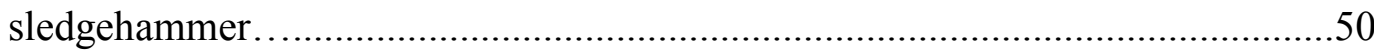

Figure 4.6 Comparison plot between signals from offset distance................................50

Figure 4.7 Comparison between the buried geophone and surface geophone- $0 \mathrm{ft}$ offset.....51

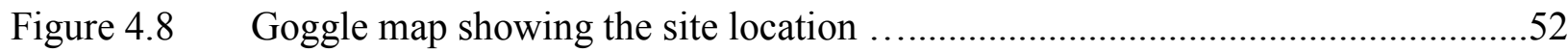

Figure 4.9 Map quest showing location of the field site...........................................52

Figure 4.10 Some of the seismic team members by the pond ..........................................53

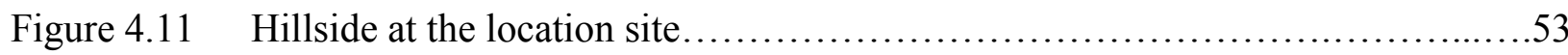

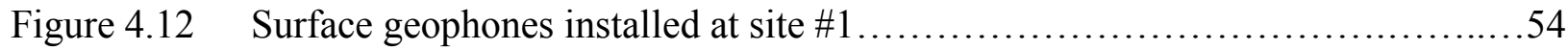

Figure 4.13 Geophone installations using a hand-held auger, close to the spillway of the pond

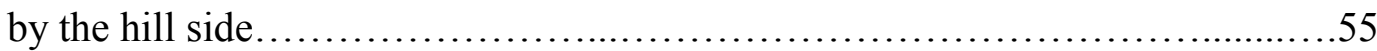

Figure 4.14 Buried geophone installations at the hillside site........................................56

Figure 4.15 Pounding location on the underground roof bolt.......................................57

Figure 4.16 Pounding location on the underground mine roof........................................57

Figure 5.1 Input portion of the EXCEL spreadsheet................................................63

Figure 5.2 Output portion of the EXCEL spreadsheet ............................................64

Figure 5.3 Signal reflection and transmission at the interfaces of a layered medium...........65

Figure 5.4 Calibrating the angle of incidence at the pounding surface.............................68

Figure 5.5 Comparison between the experimental and theoretical PPV for sledgehammer...71

Figure 5.6 Comparison between the experimental and theoretical PPV for crib bock.........72

Figure 5.7 Input section of the EXCEL program......................................................... 74

Figure 5.8 Signal waveform of the expected surface peak particle velocity......................75

Figure 5.9 Part of Input Section of the EXCEL program Federal \#2 Mine.........................76

Figure 5.10 Signal waveform of the surface peak particle velocity at Federal \#2 Mine.........77 


\section{LIST OF TABLES}

Table 3.1 Average values of Q, compressional velocity and attenuation coefficient for

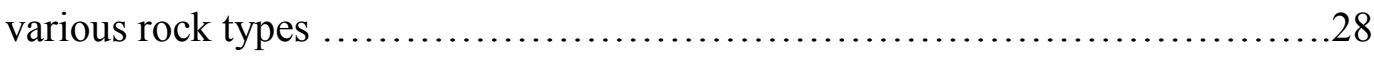

Table $3.2 \quad$ Summary of signal loss mechanisms.....................................

Table 4.0 Geological layers of the 4-West Mine of Dana Mining ........................41

Table 4.1 Location of the geophones and underground signaling sites...........................43

Table 4.2 Seismic testing at 4-West Mine of Dana Mining, underground activity log.......46

Table 4.3 Seismic results of the buried geophone at " $T$ " location on the surface...............49

Table $4.4 \quad$ Surface geophone coordinates..................................................................55

Table 5.0 The peak particle velocity, recorded for the crib block at 4-West Mine............60

Table 5.1 The peak particle velocity, recorded for the sledgehammer at 4-West Mine.....61

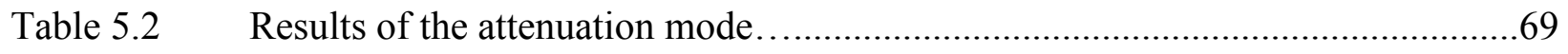

Table 5.3 Computing the vertical component of the signal energy.................................69

Table 5.4 Calculating the pounding factor of the crib block using the PPV recorded from

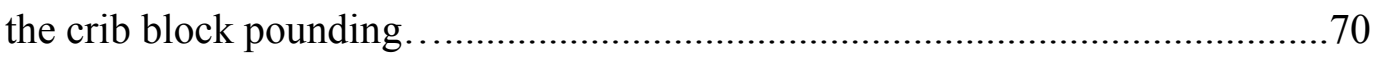

Table 5.5 Calculating the pounding factor of the sledgehammer using the PPV recorded from the sledgehammer pounding........................................................ 70

Table 5.6 Experimental and theoretical values of the PPV for sledgehammer..................71

Table 5.7 Experimental and theoretical values of the PPV for crib block.......................72 


\section{LIST OF SYMBOLS}

$\mathrm{g}(\mathrm{t}) \quad$ signal force function

$g_{o} \quad$ proportionality constant relating the surface displacement and applied force

$\mathrm{v}_{\mathrm{p}} \quad$ particle velocity

$\alpha$

coefficient of attenuation

$\kappa_{(\text {hammer })} \quad$ hammer pounding factor

$\kappa_{\text {(crib-block) }} \quad$ crib block pounding factor

$\mathrm{D}_{\mathrm{n}} \quad$ offset distance at each rock layer

$\mathrm{h}_{\mathrm{n}} \quad$ thickness of the rock layer

n nth layers

$\mathrm{N} \quad$ total number of layers

$\mathrm{v}_{\mathrm{n}} \quad$ velocity of travel within the nth layer

$r_{n} \quad$ radial distance travel within the layer

$\theta_{\mathrm{t}} \quad$ transmission angle, degree

$\theta_{\mathrm{i}} \quad$ incidence angle, degree

$\mathrm{v}_{1} \quad$ velocity of the incident medium

$\mathrm{v}_{2} \quad$ velocity of the refracted medium

$\rho \quad$ density

$\tau_{\mathrm{s}} \quad$ dwell time, sec

$\mathrm{v}_{\mathrm{p} 0} \quad$ particle velocity at the inner shell

$\mathrm{v}_{\mathrm{p} 1} \quad$ particle velocity at the outer shell

$\mathrm{r}_{0} \quad$ radius of inner shell, 


$\begin{array}{ll}\mathrm{r}_{1} & \text { radius of outer shell, } \\ \mathrm{E}_{\mathrm{k} 0} & \text { energy density at } \mathrm{r}_{0} \\ \mathrm{E}_{\mathrm{k} 1} & \text { energy density at } \mathrm{r}_{1} \\ \mathrm{~m}_{\mathrm{p}} & \text { mass of the particle } \\ t & \text { cyclic frequency of the seismic source } \\ \mathrm{m}_{\mathrm{h}} & \text { mass of the hammer } \\ \mathrm{a}_{\mathrm{h}} & \text { acceleration of the hammer } \\ \mathrm{d}_{\mathrm{h}} & \text { swing distance } \\ \mathrm{v}_{\mathrm{h}} & \text { velocity of the hammer at impact } \\ \pi & \text { pi, natural constant, 3.14159.... }\end{array}$




\section{ACKNOWLEDGMENTS}

The author wishes to express first of all his profound gratitude to God for His protection, provision and grace throughout the entire course of writing this thesis. Also I will like to express sincere appreciation to those who contributed to the content of this thesis. Particular thanks go to: Dr. Keith A. Heasley, thesis advisor, for his encouragement, advice, technical insight and most of all friendship throughout the course of this research work.

Dr. Syd S. Peng and Dr. Yi Luo for serving on the thesis committee.

The entire Faculty and Graduate students of the Mining Engineering Department of West Virginia University for their friendship and assistance and also the entire members of Redeemed Christian Church of God, Morgantown, West Virginia for their prayers Chief \& Mrs. M.O. Adebisi and Mr \& Mrs Joseph Ayeni, my parents, for encouraging my graduate education and their financial provisions.

Most importantly Oluwatoyin E. Adebisi, my wife and Adeboye and Adejare Adebisi, my sons, for their patience, support and encouragement during my graduate experience. 


\section{CHAPTER ONE}

\section{INTRODUCTION}

\subsection{General}

Coal mining is a process of removing subsurface coal resources. The two major methods which are presently used to extract coal are surface and underground mining. Compared to surface mining, underground mining is more prone to explosions and fires due to the confined working environment of a typical underground mine. In the period, 1900-2006, a total of 513 U.S. underground coal mining disasters were recorded, with most disasters resulting from explosion or fire (MSHA, 1998). In 1907, the U.S. history's worst disaster, the Monongah Mine explosion claimed 362 lives and led to the creation of Bureau of Mines and later the Mine Safety and Health Administration. In 2006, there was another mine disaster at Sago Mine that trapped 12 miners underground for several days before 11 of them eventually died and only one survived. Efforts made to rescue the miners at the Sago Mine were not successful in part due to the lack of knowledge of the miner's exact location. These trapped miners' location could not be determined because the explosion that trapped the miner had also destroyed the mine communication system. Due to not knowing the miners' location and that they were still alive, the rescue teams could not appropriately channel their efforts to save the miners' lives.

As a result of this tragic experience, the West Virginia Mine and Safety Task force in 2006 identified the need for a mine rescue seismic system that could be available for locating trapped miners if the communication/tracking system of the underground mine was damaged in a mine disaster (West Virginia Mine Safety Task Force Report, 2006).

The idea of using a seismic method to determine a trapped miner's location was first proposed by the National Academic of Engineering (National Academy of Engineering, 1970). 
In 1970 the NAE described a seismic technique that might be capable of detecting and locating a trapped miner who pounded on the roof or floor (Durkin and Greenfield, 1981). The surface particle movement that would be generated by the pounding miner was expected to be detected by geophones installed on the surface (see Figure 1.0). If this surface particle movement was detected, the underground location of the miner who generated the signal could be determined using the geophone positions and a velocity model for the overburden medium.

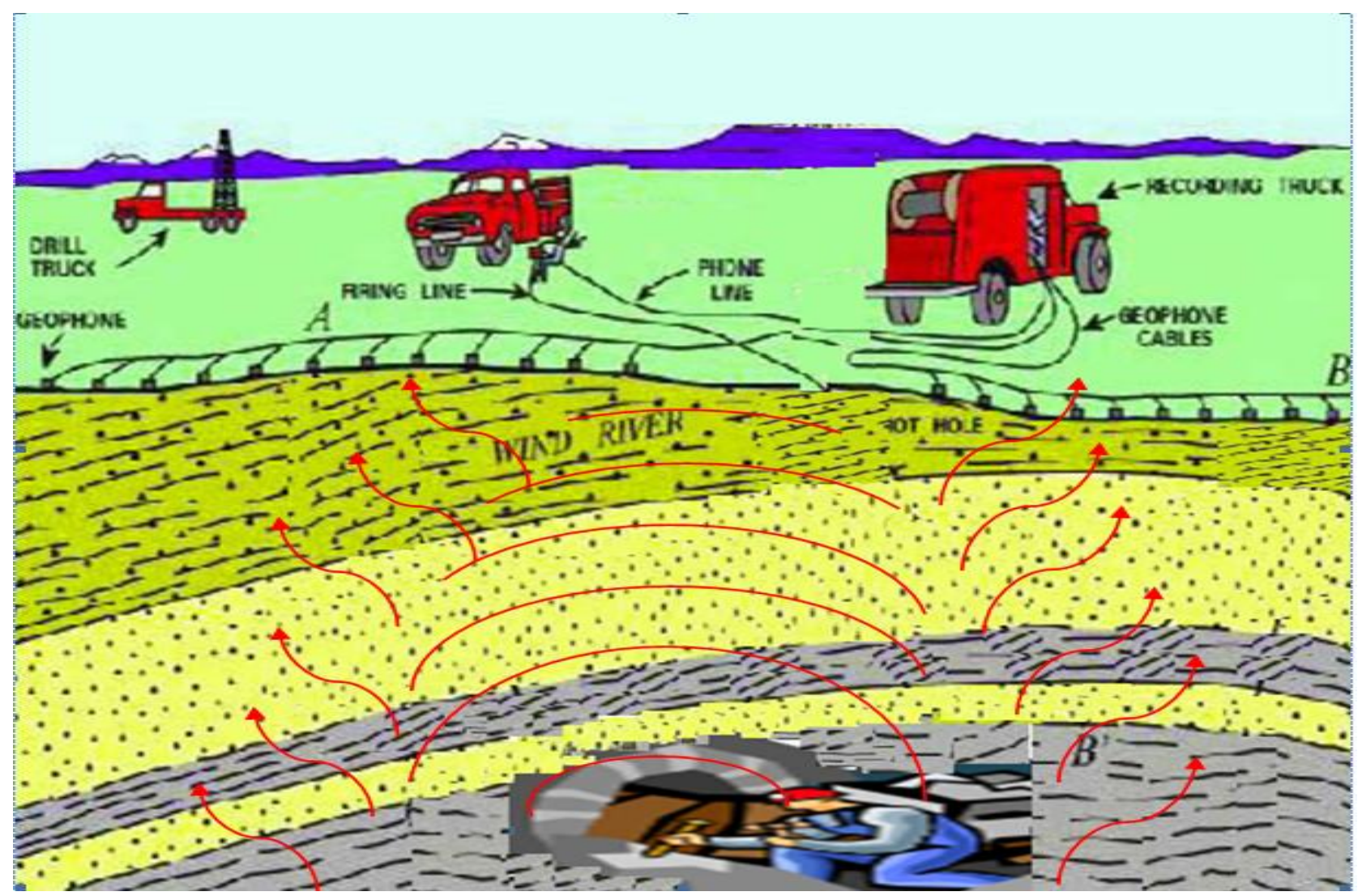

Figure 1.0. Schematic of seismic signal and propagation. (West Virginia Mine Safety Roundtable on Seismic Miner Location, 2006).

However, experiences in the past have shown that detection of the trapped miners using a mine rescue seismic system is not always guaranteed. Besides a relatively weak signal from the signaling miner, background noises, both environmental and cultural oftentimes compromises the miner's generated signal (Durkin and Greenfield, 1981). For example, vehicle movement or 
personnel activity during attempted reception of mine signals could cause interference with signal receptions. In addition, the signal being generated can be generally lost into the overburden medium. This gradual loss of the seismic energy as it moves through the overburden to the mine surface is referred to as signal attenuation.

Signal attenuation into the medium occurs through various mechanisms. One of these mechanisms is elastic hysteresis. Hysteresis is the process by which a part of the wave energy is lost into the transmitting medium through in-elastic/frictional energy dissipation, or natural damping. This loss of energy by hysteresis is frequency dependent, with higher frequencies losing greater amounts of energy over a given distance. Another mechanism through which the intensity of the seismic energy is attenuated through the geologic medium is geometric spreading. Geometric spreading is the term used to describe the process by which the signal energy per unit wave front losses energy as the wave front expands in the medium. In addition to hysteresis and geometric spreading, seismic waves can also lose part of the energy as a result of the reflection of the waves at the interfaces of each sedimentary bed or layer; most especially when the two adjacent beds have greatly different densities and compressional velocities. At the interface between sedimentary layers, part of the wave energy is reflected while the other part is refracted (transmitted) into the adjacent layer.

As a result of these signal attenuation mechanisms, the relatively weak signals resulting from the pounding miner usually becomes too weak and undetectable past a certain distance into the overburden (Heasley, 2009). Since the goal is to detect the trapped miner signal as quickly and accurately as possible, the exact extent of this limiting distance and the expected magnitude of the surface movement are considered necessary information for successful miner location. The 
ability to pre-determine these values in terms of: mine depth, geology and offset distance, would help to facilitate the optimization of future seismic deployment plans.

\subsection{Statement of Problem}

The 2006 Sago mine disaster has highlighted the need for a mine rescue seismic system that could be available for use if communication/tracking devices at a mine have been damaged by a disaster. The ultimate goal of this mine rescue system would be to detect the surface particle movement resulting from the pounding of a miner underground. However, experiences in the past have shown that detection of the miner signal is not always guaranteed, (Heasley, 2006). For instance, from our field tests performed at 4-West Mine of Dana Mining and Federal \#2 Mine of Peabody Energy, and the test performed by the state/MSHA at Consol's McElroy Mine, it was observed that the underground signals become undetectable as the signaling locations offset vertically or horizontally past a certain distance from the surface geophones, (Heasley et al, 2009). In addition, we discovered that the depth and nature of the overburden strata have a strong influence on the magnitude of this limiting distance. As a result of these observations, it is reasoned that a good understanding of the exact extent of this distance and foreknowledge about the expected magnitude of the surface peak particle movement would greatly facilitate optimizing the deployment plan for future mine rescue seismic systems.

\subsection{Scope of Work}

The major goal of this research is to provide an algorithm and implementation in an EXCEL spreadsheet program that would help to: (1) predict the expected magnitude of the surface peak particle movement for a given geology and depth, (2) provide the expected waveform of the signal, and (3) determine the exact extent of the horizontal offset distance between the pounding location and the geophones where the signal would likely become undetectable for a given mine 
depth, geology and background noise. To achieve these goals, there are three major components that need to be considered and analyzed: 1) the roof displacement resulting from the momentum of the impact of the signaling device when it hits the roof, 2) the pounding factor that relates the pounding instruments (sledgehammer or crib block) to the frequency and amplitude of the seismic signal, and 3) the attenuation of the displacement pulse as it moves through the overburden to the surface. To obtain these values, a parallel study of theoretical and experimental work was accomplished. In the theoretical analysis, a numerical model of the expected seismic signal at a given mine site was developed. To calculate the signal attenuation, the following three major signal loss mechanisms were considered:

1) Geometric Spreading,

2) Energy Dissipation by the media, and

3) Inter-bed Reflection/Transmission.

The mathematical formulas for evaluating these three signal-loss mechanisms were developed and the numerical evaluation of this formula was performed using an EXCEL spreadsheet. Once a formulation for the signal attenuation was developed, an estimate of the pounding factor of the seismic signal could then be determined by back analysis of field data. So, an attenuation model of the 4-West Mine of Dana Mining where the field test took place was performed to determine the seismic attenuation of this mine at various depths and horizontal offset distances. Then, the peak particle movements that were recorded during the seismic field experiment were backanalyzed to estimate the pounding factor at the field site. 


\section{CHAPTER TWO}

\section{LITERATURE REVIEW}

\subsection{The Physics of Seismic Waves}

For years scientists have studied the propagation of waves through the earth. From the numerous studies that have been done, a better understanding about how seismic waves pass through the earth have been achieved. For example, when a seismic signal is generated either through an earthquake, explosion or by pounding it produces elastic waves. These elastic waves are propagated through solid bodies by the transfer of forces that act upon them as an elastic deformation of the body, (Futterman, 1962). These elastic waves propagating through the earth are known as seismic waves as shown in Figure 2.0. There are several types of these seismic waves which all move and behave in different ways. One of the main types of these waves is body waves.

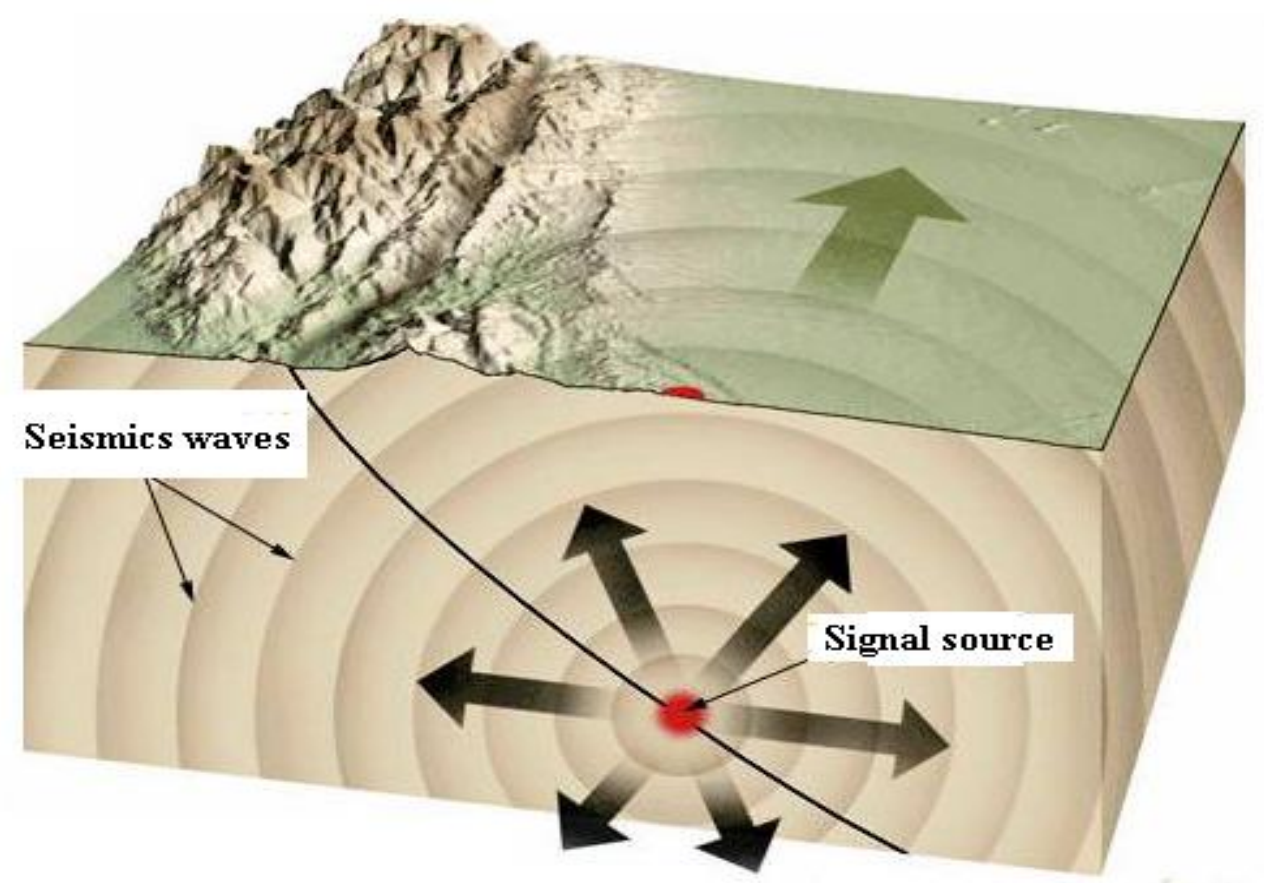

Figure 2.0. Propagation of seismic waves, ( National Geoscience Database of Iran Webpage) 


\subsection{Body Waves}

These are seismic waves that travel through the layers of the earth. They can be reflected and transmitted with density or velocity changes in the media. The propagation velocity of the body wave depends on both the elastic moduli and density of the medium as given in the equation (2.1) below:

$$
V=\sqrt{\frac{M}{\rho}}
$$

where;

$$
\begin{aligned}
& \mathrm{M} \quad=\text { elastic } \mathrm{P} \text {-wave modulus } \\
& \rho \quad=\text { density of the rock, } \mathrm{lbs} / \mathrm{ft}^{3}
\end{aligned}
$$

Body waves come in two types:

1) Compression, and

2) Shear

\section{2 Compression Waves}

This type of wave is also known as longitudinal, primary, or P-waves. Compression waves propagate by compressional and dilatational unaxial strains in the direction of wave travel. Therefore, the particle motions in P-waves oscillate about a fixed point in the same direction as the wave propagation as shown in Figure 2.1.

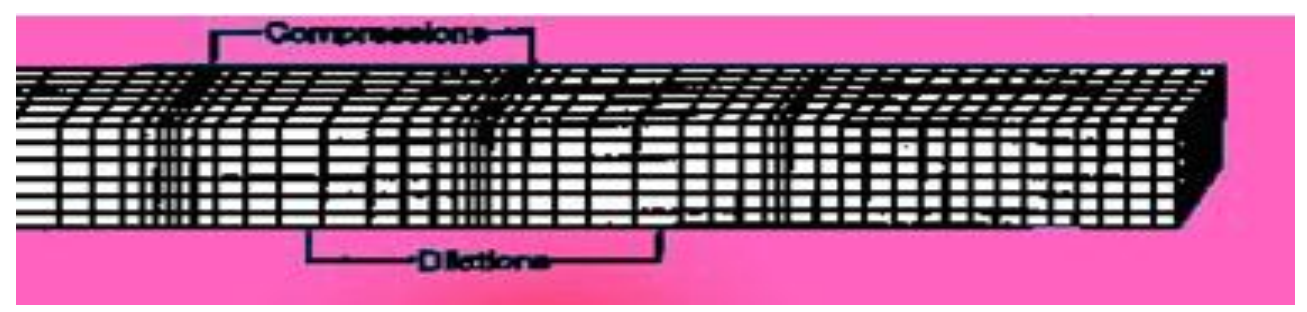

Figure 2.1. Compression (P-wave) velocity, (National Geoscience Database of Iran Webpage). 
Theoretically, the velocity of a P-wave, $\mathrm{V}_{\mathrm{P}}$, can be determined using the equation (2.2) as given below;

$$
\mathrm{V}_{\mathrm{P}}=\sqrt{\frac{\mathrm{K}+4 / 3 \mu}{\rho}}
$$

where:

$$
\begin{aligned}
& \mathrm{K} \quad=\text { bulk modulus of the media } \\
& \mu \quad=\text { shear modulus of the media } \\
& \rho \quad=\text { density of the rock }
\end{aligned}
$$

\subsection{Shear Waves}

This type of wave is also known as transverse, secondary, or S-waves. Shear waves vibrate in a direction which is perpendicular to the direction of wave travel. Therefore, the particle motions in s-waves oscillate about a fixed-point at right angles to the direction of wave propagation as shown in Figure 2.2.

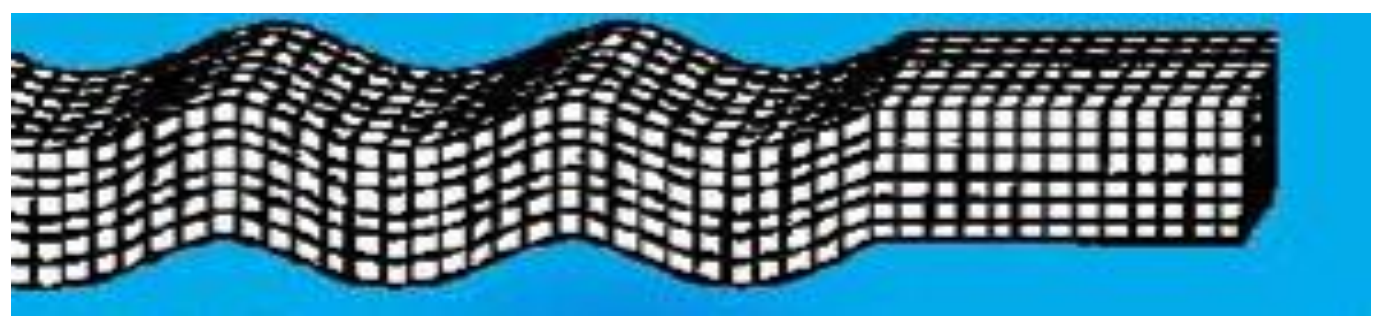

Figure 2.2. Shear (S-wave) velocity, (National Geoscience Database of Iran Webpage).

Theoretically, the velocity of a $\mathrm{S}$-wave, $\mathrm{V}_{\mathrm{S}}$, can be determined using the equation (2.3);

$$
\mathrm{V}_{\mathrm{S}}=\sqrt{\frac{\mu}{\rho}}
$$

where:

$$
\begin{aligned}
& \mu \quad=\text { shear modulus of the media } \\
& \rho \quad=\text { density of the rock }
\end{aligned}
$$




\subsection{Signal Source Generation}

Methods of seismic signal generation in mine rescue seismic systems are very different from the types that are available and applicable in oil \& gas exploration. For mineral exploration purposes, signaling devices/methods such as, explosive blasting or mechanical vibrations are a couple of the signaling techniques which can be employed (Zheng and Tsutomu, 2003). These methods deliver large amounts of energy to the ground. Whereas in a seismic location system, there are limitations to the type of signal source one can use due to the special underground coal mine environment (that is, the possible presence of explosive gases). In the underground environment, it is expected that the miner will generate a signal by pounding on the roof and/or ribs using devices for pounding which are readily available (such as a sledgehammer or crib block). To generate a seismic signal, the miner will have to produce a considerable amount of momentum to the pounding device which will impact a pulse displacement (seismic wave) on the pounding surface, (Durkin and Greenfield, 1981) as shown in Figure 2.3. According to Greenfield (1978) besides the weight of the signaling devices, the velocities of pounding as well as the nature of the pounding surface also have some influences on the magnitude of signal being generated.

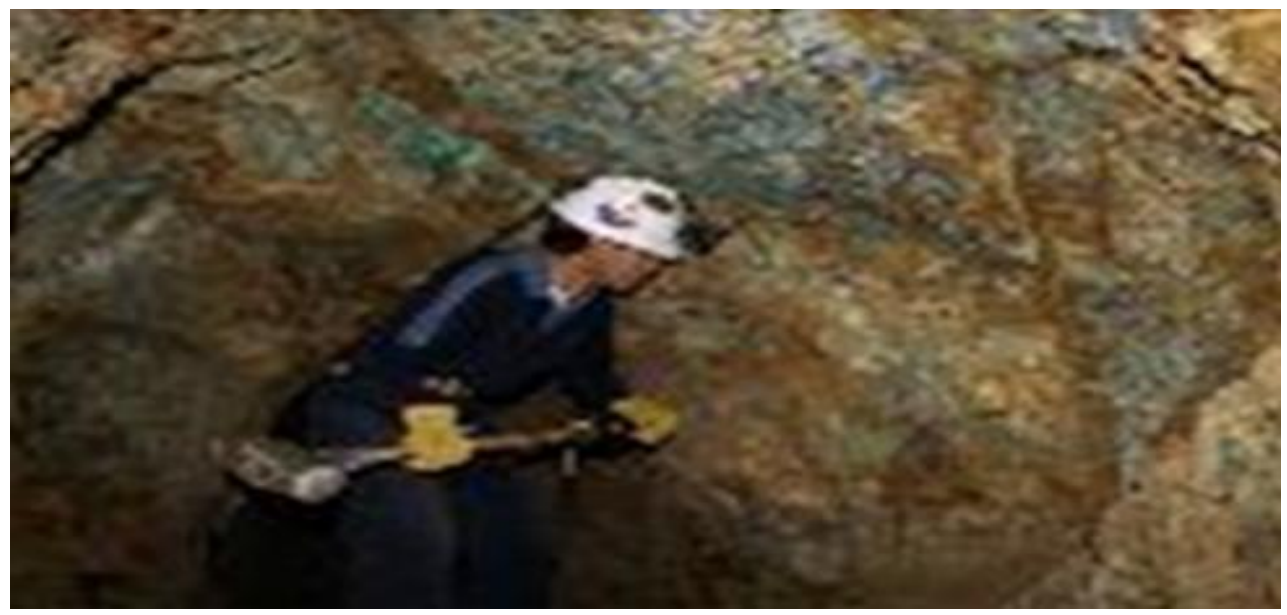

Figure 2.3. Pounding with a sledgehammer, (Institute of Physics Webpage) 


\subsection{Numerical Representation of Signal Source}

Various researchers in the past have tried to derive a numerical representation for a point source signal using different approaches. Though the approaches may vary they all generally make the same basic assumptions

1. It is generated by an impulsive force (Kuo, 1974)

2. The generated waves are time varying and sinusoidal (see Figure 2.4) (Greenfield, 1978)

3. The generated waves decays rapidly with time (Heasley, 2009)

Using the basics assumptions stated above, the researchers were able to develop a numerical representation of a typical signal source having the waveform as shown in Figure 2.4. At the point of impact, the particle velocity at the pounding location increases to the peak value and then starts to decay rapidly with time.

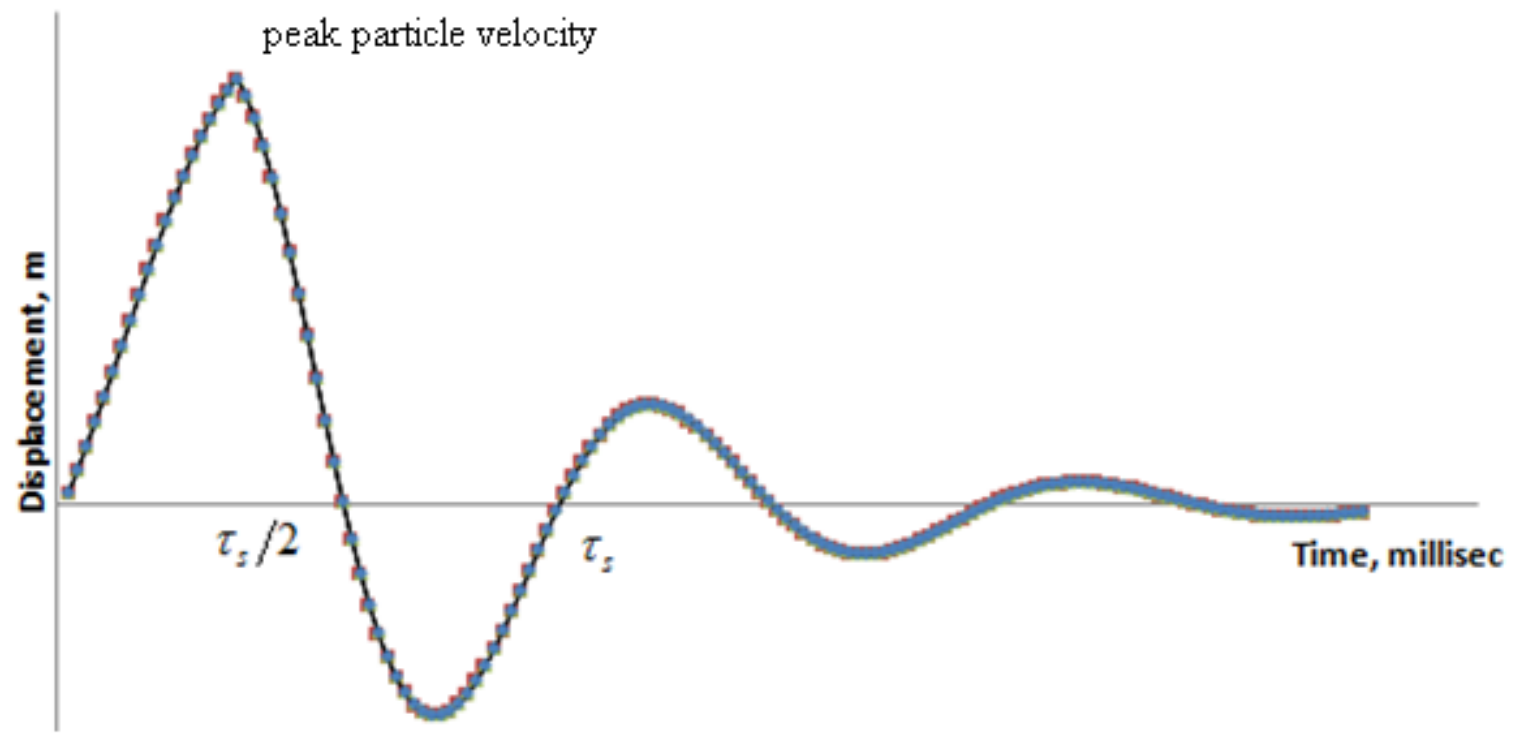

Figure 2.4. Waveform of a typical signal source generated by an impulsive force. 


\subsection{Particle Velocity}

The term "particle velocity" refers to the velocity, $v$, of a particle in the medium as the particle vibrates and transmits the seismic wave from the signal source to the geophones installed on the surface. This is not the same as the propagation velocity of a seismic wave with which the seismic energy travels through a medium. In the case of compression waves, their propagation velocity through rocks is of the order of a few thousand meters per second. However, in the case of particle velocities, the value may be as small as $10^{-6}$ meter per second and involve ground displacements as little as $10^{-10} \mathrm{~m}$.

The detection of seismic waves traveling through the earth involves measuring these very small ground displacements and particle velocities typically by using a geophone.

\subsection{Geophone}

In order to detect the vibration of the earth, a very sensitive device known as geophone is employed. The geophone is made up of: a mass, a coil, the case, a permanent magnet, a spring and typically a planting spike. The mass together with a wire coil is suspended by a spring within the magnetic field (flux, $\phi$ ) (see Figure 2.5). This magnetic field is created by the permanent magnet attached to the geophone case that houses the entire components of the geophone, (Krohn, 1984). To ensure that the geophone is firmly coupled to the ground and faithfully follows the displacement motion of the ground, a planting spike is fixed at the base of the geophone.

As the planted geophone faithfully follows the ground motion, it also causes an equal effect to the coil on the spring to oscillate at a velocity considered to be proportional to ground velocity. By applying Faraday law, the relative motion between the coil and the case produces 
voltage that is proportional to the coil velocity. The mathematical representation of the geophone operation employing Faradays Law can be described as given in the equation (2.4);

$$
\mathrm{e}=\mathrm{Gv}
$$

where:

e $\quad=$ output voltage of the geophone

$\mathrm{G}=$ the generator constant of the geophone

$\mathrm{V}=$ displacement motion of the ground (assume to be proportional to the ground velocity)

Going by the above mathematical equation, one can easily conclude that output voltage of the geophone is proportional to the ground particle velocity. A typical single geophone is only sensitive to vibration along a single axis.
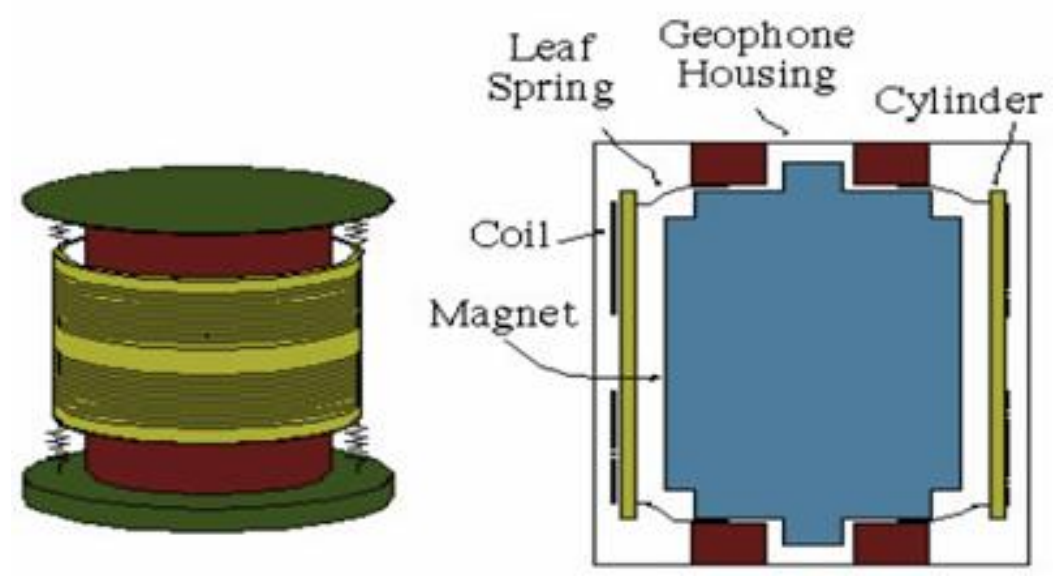

Figure 2.5. Geophone components, (West Virginia Mine Safety Roundtable on Seismic Miner Location, 2006).

\subsubsection{Unidirectional and Multidirectional Geophones}

A multidirectional geophone has more than one coil oriented at different angles such that it can detect seismic signal from both horizontally waves as well as vertically waves. A typical example of this type of geophone is tri-axial geophone (see Figure 2.6). In a triaxial geophone, 
each singular geophone is in an orthogonal direction to each other thereby making it a three component geophone. These three component geophones are usually packaged in tubes with three or more single geophones in $\mathrm{X}, \mathrm{Y}$ and $\mathrm{Z}$ axis and wired together. For unidirectional geophones, the geophone is oriented in one direction and can only respond to signal in the oriented direction (Wolf, 1941).

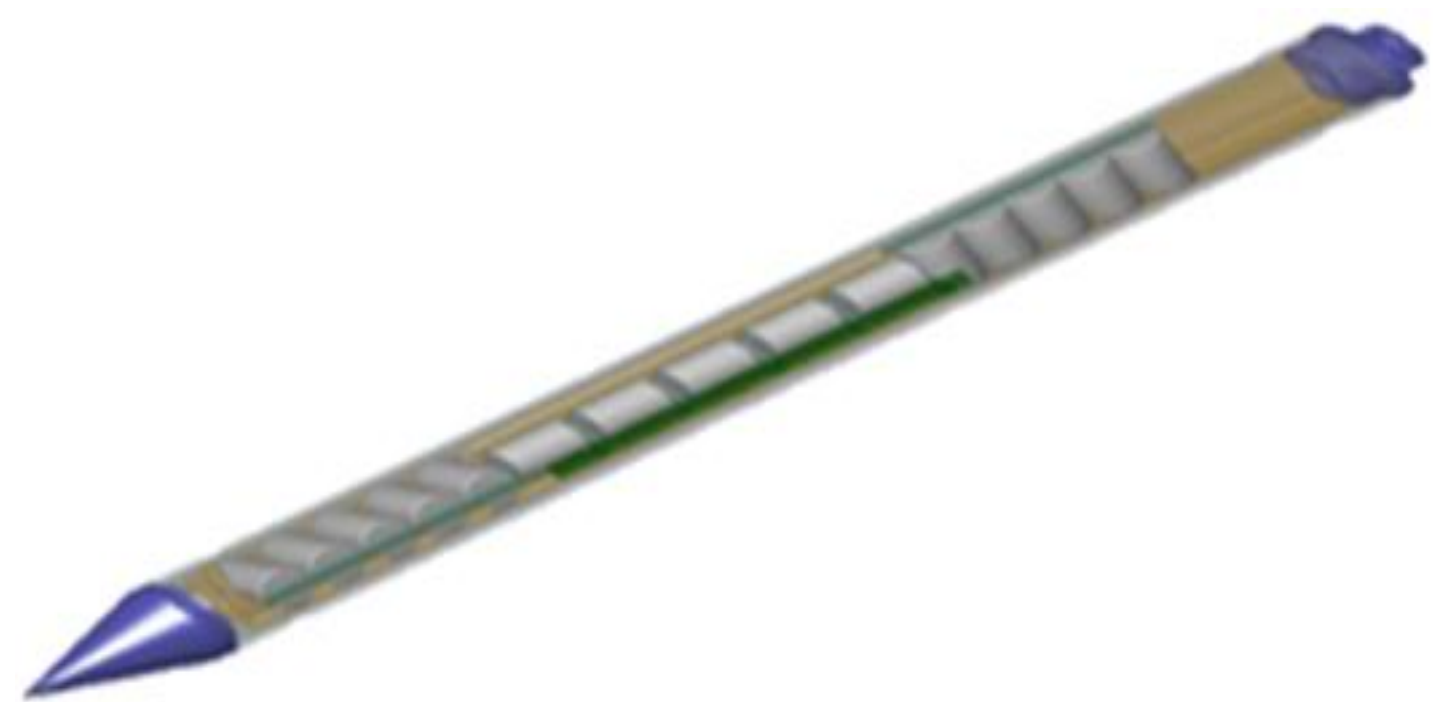

Figure 2.6. Three components geophone, (West Virginia Mine Safety Roundtable on Seismic Miner Location, 2006).

\subsection{Coal Mine Event Location Studies}

Under contract with the U.S Bureau of Mines, the Westinghouse Electric Corporation conducted a study to develop and implement seismic techniques for the location of trapped miners. In 1972, the first seismic rescue equipment (see Figure 2.7) was designed and assembled, followed with a series of experimental studies at several mines. The main purposes of these studies were to determine the best techniques for data collection which maximize the signal, and also to analyze the performance of the equipment. 


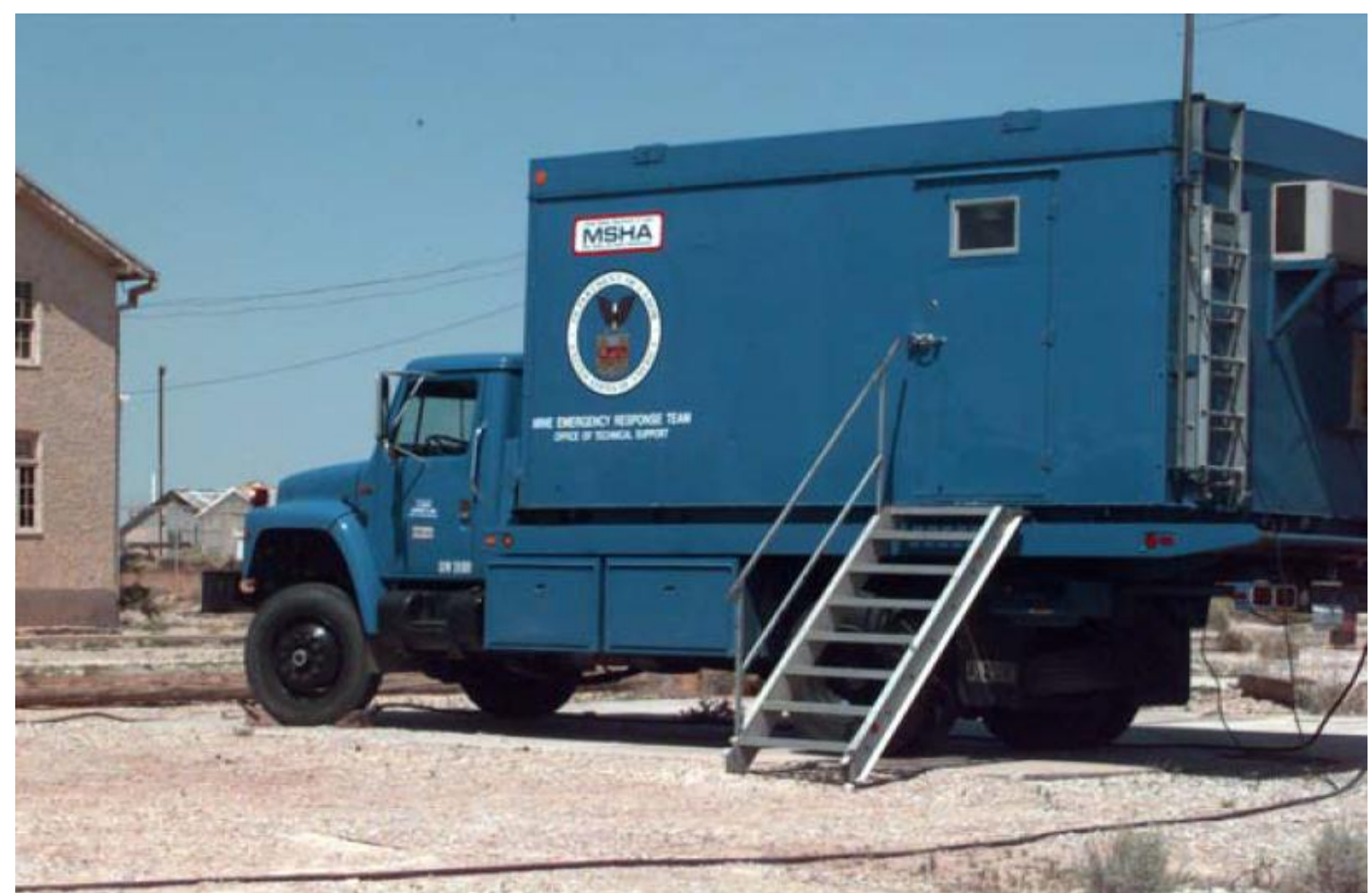

Figure 2.7. Westinghouse equipment truck, (West Virginia Mine Safety Roundtable on Seismic Miner Location, 2006).

The Westinghouse Electric Corporation Coal Mine Rescue and Survival, (1971) concluded that heavy sources of large cross-section give the largest signals, and that burying of the geophones was critical to noise minimization. It was also found that the use of symmetric multiple geophone sub arrays instead of single sensors reduced random noise substantially and reduced coherent noise to a lesser degree by spatial filtering.

In another study sponsored by the U.S Bureau of Mines and conducted by Arthur D. Little, Inc. (1974) evaluated the state of the art of the seismic miner location procedure. In one chapter of the report, the researcher analyzed detection range as a function of source type and of various noise levels. It was found that detection is possible for slant ranges (source to receivers distances) on the order of $1000 \mathrm{ft}$ where conventional $\mathrm{S} / \mathrm{N}$ (signal to noise ratio) improvement techniques are used under usual seismic noise conditions without the presence of any man-made noise. 
In order to minimize the discrepancy between the assumed velocity model and the true earth it represents, a general knowledge of the geology to be expected in the vicinity of mines would be beneficial. A chapter of the Arthur D. Little report addressed the question of possible earth models and concluded that, in U.S coal mining regions, geologic strata are usually horizontal (a slope of $100 \mathrm{ft} / \mathrm{mile}$ would be unusually large) and that the general characteristics of the geologic cross sections persists for distances of several miles. Compressional velocities may range from that of the weathered layer (as low as $500 \mathrm{ft} / \mathrm{sec}$ ) to that of limestone (as high as $14,000 \mathrm{ft} / \mathrm{sec}$ ) as shown in Figure 2.8 below. Therefore a laterally homogenous, horizontally layered medium would seem to be a viable candidate for a representative earth model. The study warns, however, that such a model does not take into account the variable depth of the weathered layer below the geophones and, hence, its use may result in location errors. A good refraction survey is suggested as a preliminary step to the development of an appropriate velocity model.

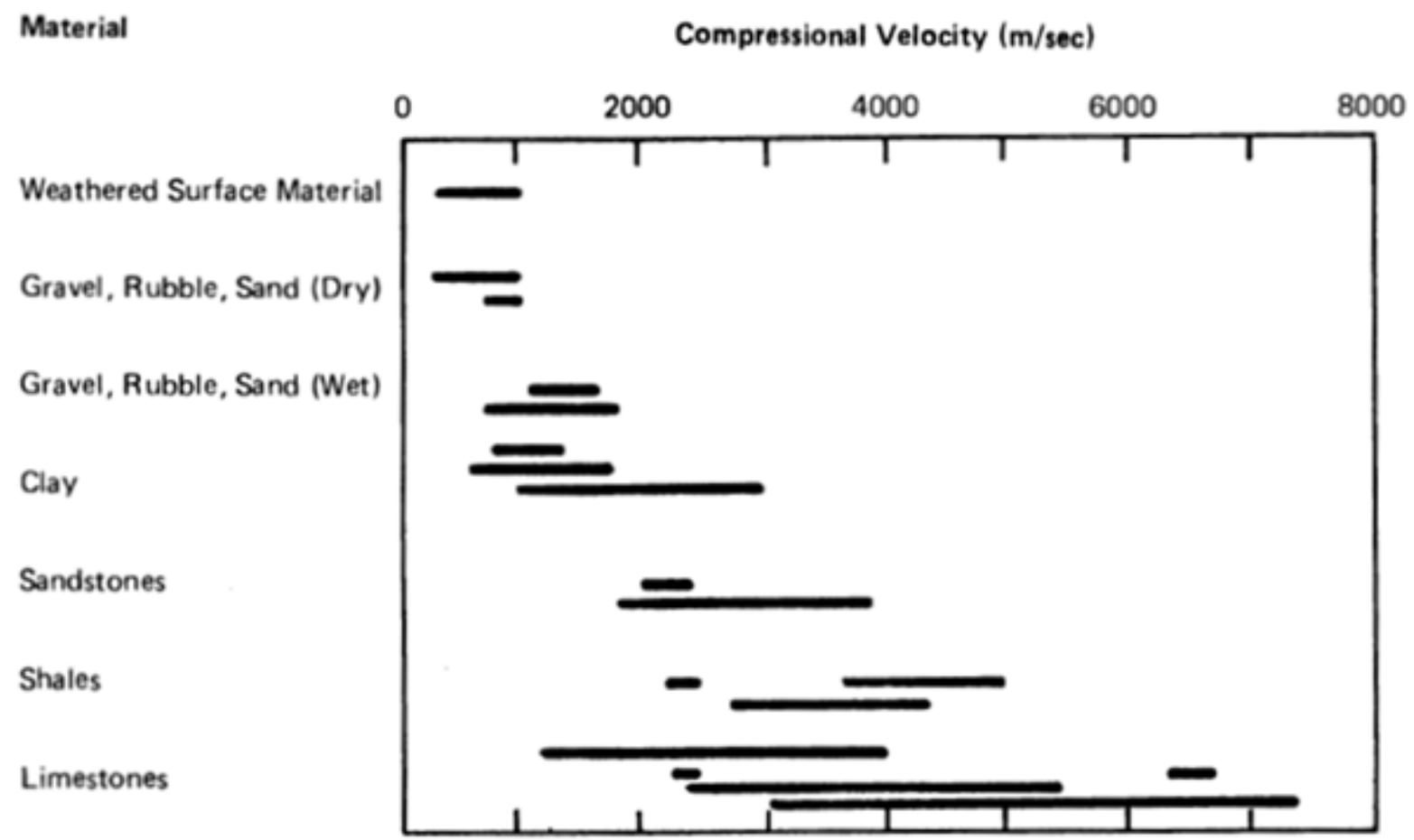

Figure 2.8. Compressional velocity of various sedimentary materials, (Arthur, 1974). 
In 1976, J. Powell and J. Watson conducted a study to determine the possibility of using an in-mine geophone seismic system. In contrast to a typical mine rescue seismic system; the geophones of the in-mine geophones seismic system are attached to a roof bolt in the mine. The purpose of this in-mine geophone installation was to eliminate the passage of the seismic signal through the overburden strata but rather have the seismic signal travel through wires that connect the geophones in the mine to the seismic data receiving station on the surface. After testing of this technique at a Research Mine in Bruceton, PA., the author identified the process of installing geophones in the mine as one of the possible ways of minimizing attenuation. However, the problem with an in-mine seismic system is that the connecting wires are vulnerable to a subsequent mines fire or explosion just like the in-mine communication equipment.

In another study done by Kuo (1974), the author discussed the possibility of generating a low-frequency seismic source. The rate of attenuation of this type of seismic source is relatively low when compared with a seismic source with a high frequency. According to seismic attenuation theory, the rate of attenuation is directly proportional to the frequency content of the signal source; hence, a signal source with a high frequency tends to attenuate more than a source with a low frequency. When trying to achieve a low frequency, signal source, the author considered using a lead sphere because of its weight advantage, (since the mass of a pounding device is proportional to the magnitude of the particle displacement resulting from the pounding, (Kuo, 1974). Also, in order to minimize the energy loss through heat dissipation and plastic deformation at the pounding surface, the author considered the idea of installing a coupler in the form of a spring at the point of pounding. With this type of seismic signal generation, the author was able to successfully generate a seismic signal with a low frequency. However, the heavy 
lead weight and spring at the point of pounding may not always be available for use after a mine explosion or fire.

In another investigation carried out by Powell and Watson (1976), the authors derived two equations that might calculate the surface peak particle movement at a particular mine. During this study, a couple of seismic field experiments were performed from which the author derived the equations. The first equation was the modification of the surface peak particle movement equation that was once suggested by Bureau of Mines investigator when explosives were used as the signal source. The Bureau of Mines investigator in this investigation assumed that the surface peak particle movement of the seismic waves decayed in the order given in the equation (2.5) below:

$$
\mathrm{R}^{-\mathrm{a}}
$$

where:

$$
\begin{aligned}
& \mathrm{R} \quad=\text { distance from the source, } \mathrm{ft} \\
& \mathrm{a} \quad=\text { constant in the range of 1.2-1.6. }
\end{aligned}
$$

Powell and Watson (1976), through their field testing, modified this Bureau of Mines equation with the experiment data by deriving a value for the constant a based on the mine geology and geophone location. The calculated value for a using a least-square fit method showed that the optimal value for a is between 1.9 and 2.6 with a standard deviation on the order of 0.1 . The magnitudes of the surface peak movement obtained from this equation were appreciably higher than those in the field testing. As a result of these observations, the authors went further to empirically derive a second equation by plotting the experimental data taken from the seismic field experiments. From the graph, the author obtained a better fit to the experimental field data while assuming the seismic signal decay was proportional to equation (2.6): 


$$
\frac{e^{-c R}}{R^{\frac{1}{2}}}
$$

where:

c $\quad=$ constant which depends on the mining height and the geophone placement.

$\mathrm{R}=$ distance from the seismic source.

According to the report, this second equation was able to predict the signal strength better than the first equation. However, this equation suffers from different shortcomings which limit its versatility and the area of application. One of the shortcomings is that the experiment did not incorporate the methodology of a typical seismic location system into its scheme, such as, the use of a typical signaling device. In addition, the effect of the mine seismic properties and other source of signal loss mechanism, such as, dissipation, geological layering of the overburden were also not directly considered when deriving the equation. The other shortcoming is that the signal processing equipment to process the experimental data which was used to derive the equation was out of date. Given the number of shortcomings, the application of the equation to modern seismic location systems is largely questionable.

As a way to further improve the equations which were derived by Watson and Powell, the Bureau of Mines conducted a series of seismic field experiments at Copper Queen Mine (Durkin and Greenfield,1981). In these experiments, typical seismic signal methods and devices were employed such as generating the seismic signal by pounding on the mine roof or ribs. In order to derive a suitable equation that could describe the magnitude and waveform of a surface particle movement (resulting from a pounding miner), the researchers at the Bureau of Mines modified a seismic source theory equation which was originally derived by White (1965). White (1965) 
gives the displacement due to the compressional velocity which creates the radial component of the displacement, $d_{r}(t)$, for a point source in an infinite medium as given in equation (2.7);

$$
d_{r}(t)=\frac{\cos \theta}{4 \pi \rho V^{2}} \times \frac{1}{R} \times g\left(t-\frac{R}{V}\right)
$$

where:

$$
\begin{array}{ll}
\theta \quad & =\text { the angle between the source-receiver direction and the vertical } \\
\rho & =\text { the density of the roof material } \\
\mathrm{R} & =\text { the radial distance between the source and the receiver } \\
\mathrm{V} & =\text { the P-wave velocity of the medium. } \\
g\left(t-\frac{\mathrm{R}}{\mathrm{V}}\right) & =\text { signal Force-time function at the time the signal gets to a receiver }
\end{array}
$$

In this equation, the geometric spreading is included with the $1 / \mathrm{R}$ term. This geometric spreading is the signal energy per unit wave front loss as the wave front expands in the medium. The (t$\mathrm{R} / \mathrm{V}$ ) term offsets the time variable by the length of time it takes the signal to travel from the source to the receiver (distance divided by the velocity). 


\section{CHAPTER THREE}

\section{CALCULATING THE SURFACE SEISMIC SIGNAL STRENGTH}

\subsection{Introduction}

In order to determine the magnitude of the surface peak particle movement (displacement or velocity) resulting from a pounding miner in a mine rescue seismic system, there are three major components which need to be considered. These three components are;

1) The roof displacement resulting from the momentum of the impact of the signaling device when it hits the roof.

2) The pounding factor that relates the pounding instruments (sledgehammer or crib block) to the frequency and amplitude of the seismic signal (by the sledgehammer or crib block), and

3) The attenuation of the displacement pulse as it moves through the overburden to the surface To calculate the signal attenuation, the following three major signal loss mechanisms need to be considered:

1) Geometric Spreading,

2) Energy Dissipation by the media and

3) Inter-bed Reflection/Transmission (see Figure 3.0).

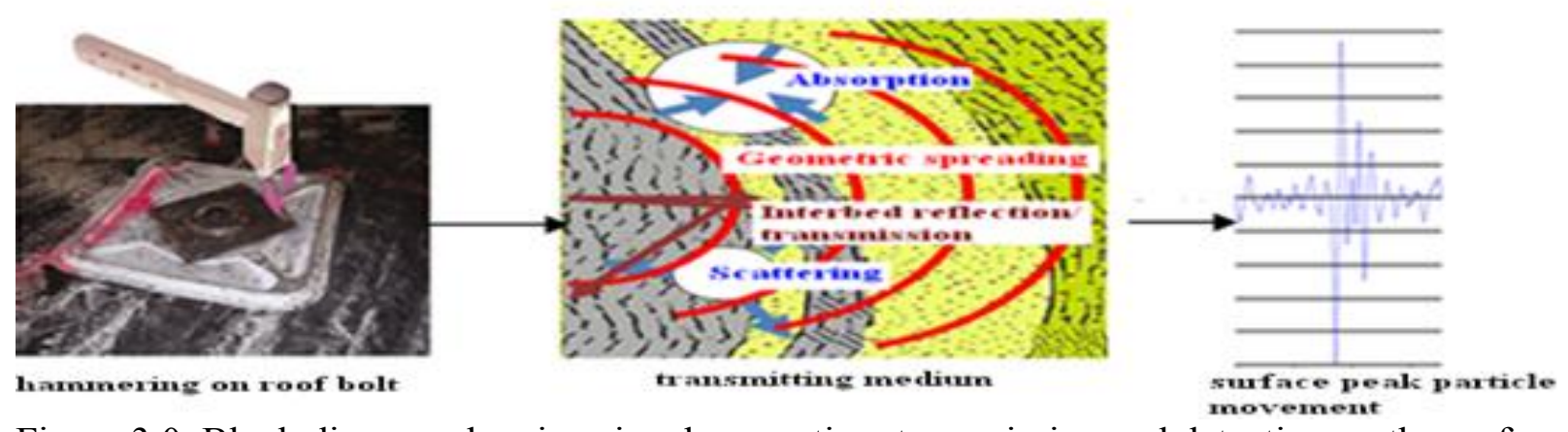

Figure 3.0. Block diagram showing signal generation, transmission and detection on the surface. 


\subsection{Signal Source}

In a seismic location system, the signal source is generally achieved by the miner pounding on the coal roof, coal floor or roof bolt, (see Figure 3.1). The wave generated by the pounding has the following characteristics, (Durkin and Greenfield, 1981)

1) It is generated by an impulsive force (Kuo, 1974)

2) It is time varying (Greenfield, 1978)

3) It decays (rapidly) with time (Heasley, 2009)

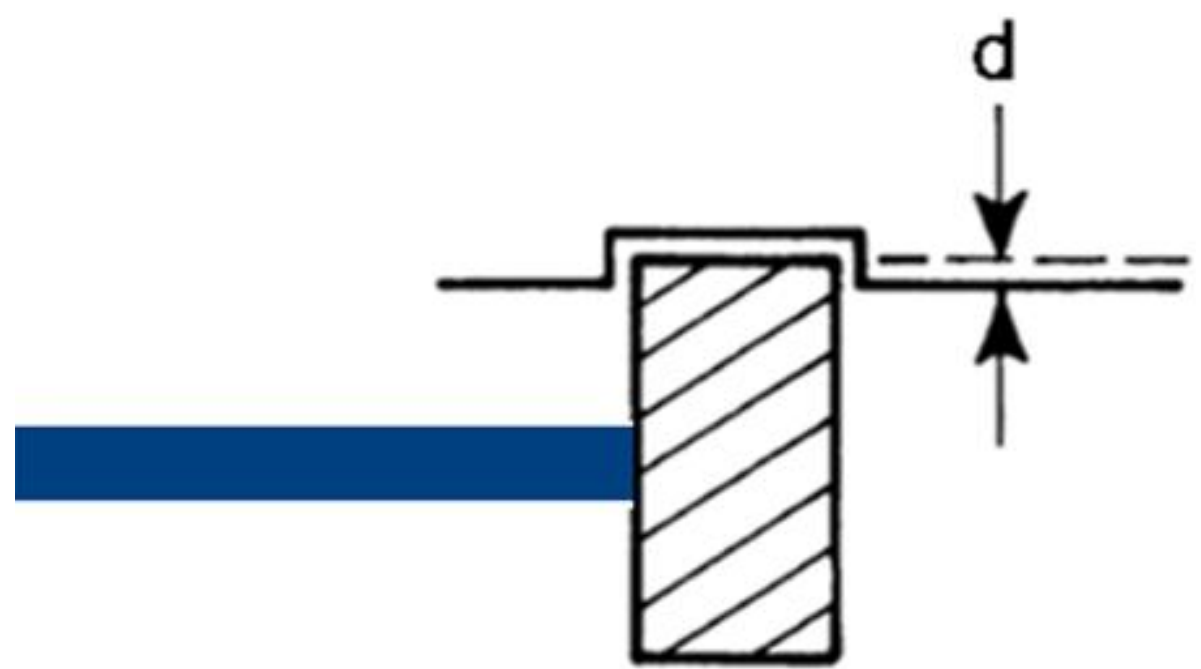

Figure 3.1. A typical miner's signal source, (Kuo, 1974).

3.1.1 Signal Force-Time Function: In order to derive the signal force function for a practical situation, it is imperative to use the above characteristics as general guidelines. First, the signal force as a function of time, $\mathrm{g}(\mathrm{t})$, generated at the pounding's location by the signaling devices would be proportional to the displacement-time function of the pounding surface, $d(t)$, of the pounding surface (Kuo, 1974):

$$
g(t)=g_{o} d(t)
$$


where:

$\mathrm{g}(\mathrm{t}) \quad=$ the signal force-time function

$g_{O} \quad=$ the proportionality constant relating the surface displacement and applied force

3.1.2 Displacement time function: displacement time function, $d(t)$ of the pounding surface can be best represented by a sine function with an exponential decay term as (Sung, 1953):

$$
\mathrm{d}(\mathrm{t}) \approx \sin \frac{\pi \mathrm{t}}{\tau_{\mathrm{S}} / 2} \times \mathrm{e}^{-\mathrm{at}}
$$

where:

$\mathrm{d}=$ the displacement of the pounding surface, $(\mathrm{m})$

$\tau_{s} \quad=$ the dwell time of signaling device at the pounding surface (and cycle time)

A = the value that determines the rate at which the impulse decays with time,

Substituting equation (3.2) into equation (3.1) we get:

$$
\mathrm{g}(\mathrm{t}) \approx \mathrm{g}_{0} \times \sin \frac{\pi \mathrm{t}}{\tau_{\mathrm{S}} / 2} \times \mathrm{e}^{-\mathrm{at}}
$$

The general shape of this function is shown in Figure 3.2. The peak displacement of the surface particle occurs during the half time of the dwell time and begins to decay at a particular rate. The rate of decay, a, is largely depend on the nature of the pounding surface. 


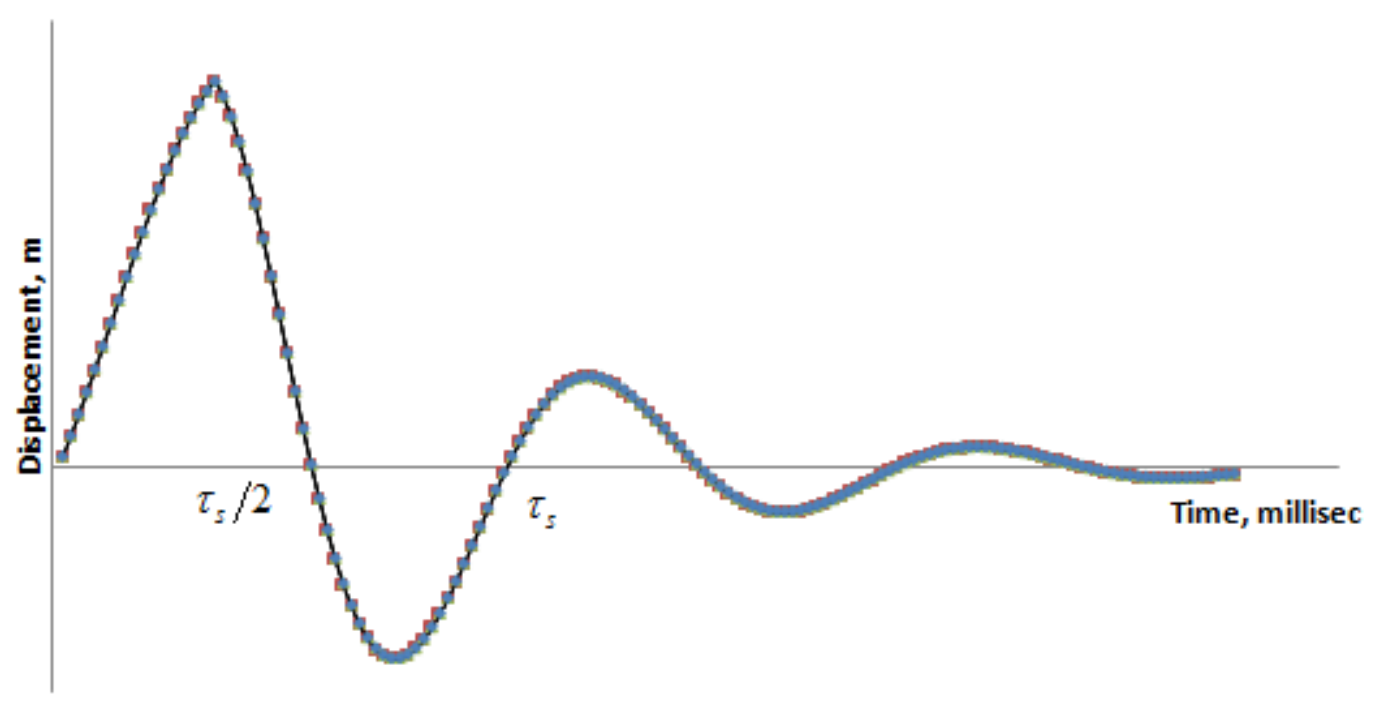

Figure 3.2. A typical waveform pattern of a miner's signal.

In general, we are only interested in the peak particle movement which occurs during the first half cycle at time $<=\tau_{\mathrm{s}} / 2$. In this first half cycle, the decay can be considered negligible, therefore equation (3.3) can be re-written as:

$$
\begin{aligned}
\mathrm{g}(\mathrm{t}) & =\mathrm{g}_{0} \times \sin \frac{\pi \mathrm{t}}{\tau_{\mathrm{s}} / 2} \text { for } 0 \leq \mathrm{t} \leq \tau_{\mathrm{s}} / 2 \\
& =0 \text { otherwise }
\end{aligned}
$$

For the force-time function to be useful in the analysis of the seismic waves, it is imperative that the proportionality constant, $\mathrm{g}_{0}$, that relates the surface particle displacement with the pounding force, be determined as exactly as possible. This proportionality constant can be determined by assuming conservation of momentum in the impact, such that the momentum of the impact of the hammer when it hits the roof equals the momentum imparted to the roof (Heasley, 2009).

3.1.3 Hammer Momentum: For the hammer, the work done in accelerating the hammer is equal to the kinetic energy at the time of impact as given in equation (3.5): 


$$
\mathrm{m}_{\mathrm{h}} \mathrm{a}_{\mathrm{h}} \times \mathrm{d}_{\mathrm{h}}=\frac{1}{2} \mathrm{~m}_{\mathrm{h}} \mathrm{v}_{\mathrm{h}}^{2}
$$

where:

$$
\begin{aligned}
& \mathrm{m}_{\mathrm{h}} \quad=\text { mass of the hammer } \\
& \mathrm{a}_{\mathrm{h}} \quad=\text { acceleration of the hammer } \\
& \mathrm{d}_{\mathrm{h}} \quad=\text { swing distance } \\
& \mathrm{v}_{\mathrm{h}} \quad=\text { velocity of the hammer at impact }
\end{aligned}
$$

Solving for the velocity of the hammer at impact, $v_{h}$, we find:

$$
\mathrm{v}_{\mathrm{h}}=\sqrt{2 \mathrm{a}_{\mathrm{h}} \mathrm{d}_{\mathrm{h}}}
$$

And the momentum of the hammer, $\mathrm{P}_{\mathrm{h}}$, which is equal to the mass times the velocity is:

$$
P_{h}=\sqrt{2 m_{h}^{2} a_{h} d_{h}}
$$

3.1.4 Roof Momentum: To determine the momentum imparted to the roof, the time integral of equation 3.4 for the first half cycle needs to be determined:

$$
\int_{0}^{\tau_{\mathrm{s}} / 2} \mathrm{~g}(\mathrm{t}) \mathrm{dt}=\mathrm{g}_{0} \int_{\mathrm{o}}^{\tau_{\mathrm{s}} / 2} \sin \frac{\pi \mathrm{t}}{\tau_{\mathrm{s}} / 2} \mathrm{dt}
$$

Integrating

$$
\begin{aligned}
\mathrm{g}_{0} \int_{\mathrm{o}}^{\tau_{\mathrm{s}} / 2} \sin \frac{\pi \mathrm{t}}{\tau_{\mathrm{s}} / 2} \mathrm{dt} & =\mathrm{g}_{0} \frac{\tau_{\mathrm{s}}}{2 \pi}\left[-\cos \frac{\pi \mathrm{t}}{\tau_{\mathrm{s}} / 2}\right]_{0}^{\tau_{\mathrm{s}} / 2} \\
& =\mathrm{g}_{0} \frac{\tau_{\mathrm{s}}}{2 \pi}[-\cos \pi-(-\cos 0)] \\
& =\mathrm{g}_{0} \frac{\tau_{\mathrm{s}}}{\pi}
\end{aligned}
$$


3.1.5 Proportionality Constant: To solve for the proportionality constant, $g_{0}$, equation (3.7) is set equal to equation (3.9) and then solved for $\mathrm{g}_{0}$ :

$$
\mathrm{g}_{0} \frac{\tau_{\mathrm{s}}}{\pi}=\sqrt{2 \mathrm{~m}_{\mathrm{h}}^{2} \mathrm{a}_{\mathrm{h}} \mathrm{d}_{\mathrm{h}}}
$$

Therefore:

$$
\mathrm{g}_{0}=\frac{\pi}{\tau_{\mathrm{s}}} \sqrt{2 \mathrm{~m}_{\mathrm{h}}^{2} \mathrm{a}_{\mathrm{h}} \mathrm{d}_{\mathrm{h}}}
$$

\subsection{Signal Attenuation}

Signal attenuation is the gradual loss of signal energy through the transmitting medium as seismic waves travel from the source to receivers (geophones) as shown in Figure 3.0. The following are the 3 major signal loss mechanisms that need to be considered:

1) Energy Dissipation by the media,

2) Geometric Spreading, and

3) Inter-bed Reflection/Transmission.

3.2.1 Energy Dissipation: This is the process by which a part of the wave energy is lost into the transmitting medium through dissipation (elastic hysteresis, natural damping). Dissipation is the concept of a dynamical system where waves lose energy over time, typically due to the action of friction. The lost energy is typically converted into heat, raising the temperature of the system, (Knopoff and MacDonald, 1962). In order for the signal loss due to the heat dissipation to be determined, a dimensionless measure of energy dissipation $1 / Q$ (inverse is the material quality factor) is defined as,

$$
\frac{1}{Q}=\frac{\Delta E}{4 \pi E}
$$


where:

$$
\begin{aligned}
& 4 \pi \quad=\text { the unit surface area of the material medium } \\
& \mathrm{E} \quad=\text { the elastic energy. } \\
& \Delta E \quad=\text { the loss of energy (in one cycle) }
\end{aligned}
$$

The quality factor, Q, quantifies the ability of a rock to attenuate seismic waves. Rocks are found to have values in the range $10-400$ with the typical sedimentary rock having a value near 100 (Durkin and Greenfield, 1981), (see Table 3.1). The value 1/Q is known as the damping ratio (D) of the material medium and is the ratio of energy loss over total energy for a single vibration cycle. One of the characteristics of signal energy dissipation as heat into the propagating medium is that it is frequency dependent. A seismic wave at a higher frequency tends to dissipate more energy than the waves at a lower frequency. According to Futterman, (1962), the coefficient of attenuation, $\alpha$, is related to the $\mathrm{Q}$ value of the transmitting medium and the angular frequency $\omega$ of the seismic waves as:

Solving for $\alpha$;

$$
\mathrm{Q}^{-1}(\omega) \approx \frac{2 \alpha \mathrm{V}}{\omega}=\frac{2 \alpha \mathrm{V}}{\pi \mathrm{f}}
$$

$$
\alpha=\frac{\omega}{2 V Q}=\frac{\pi f}{2 V Q}
$$

where:

$$
\begin{array}{ll}
\alpha & =\text { coefficient of attenuation } \\
t & =\text { cyclic frequency of the seismic source, cycle/sec } \\
\mathrm{V} & =\text { seismic velocity of the wave }
\end{array}
$$

Equation (3.14) implies that signal energy dissipation as heat into the propagating medium is directly proportional to the frequency of the seismic waves. This Coefficient of Attenuation is then implemented such that the signal strength of the seismic waves decays exponentially as the waves move away from the signal source: 


$$
\mathrm{E}_{1}=\mathrm{E}_{0} \times \mathrm{e}^{-\alpha \mathrm{R}}
$$

where:
$\mathrm{E}_{0} \quad=$ the energy level at the source
$\mathrm{E}_{1} \quad=$ the energy level at some distance, $\mathrm{R}$, from the source
$\mathrm{R}=$ the radial distance from the source to the location of $\mathrm{E}_{1}$

Table 3.1. Average values of $\mathrm{Q}$, compressional velocity and attenuation coefficient for variuos rock types (Durkin and Greenfield, 1981).

\begin{tabular}{|c|c|c|c|}
\hline Rock type & $\begin{array}{c}\text { Compressional } \\
\text { velocity, ft/sec }\end{array}$ & $\begin{array}{c}\text { Rock } \\
\text { Quality } \\
\text { factor }\end{array}$ & $\begin{array}{l}\text { Attenuation } \\
\text { coefficient, nepers/ft }\end{array}$ \\
\hline Dolomite & 16,000 & 200 & $9.82 \times 10^{-5}$ \\
\hline Limestone & 14,000 & 120 & $1.87 \times 10^{-4}$ \\
\hline Sandstone & 8,000 & 50 & $7.85 \times 10^{-4}$ \\
\hline Shale & 10.000 & 50 & $6.28 \times 10^{-4}$ \\
\hline $\begin{array}{c}\text { Weathering } \\
\text { zone }\end{array}$ & 4000 & 15 & $5.24 \times 10^{-3}$ \\
\hline
\end{tabular}

3.2.2 Geometric Spreading: Geometric spreading is the term used to describe the process by which the signal energy per unit wave front decreases. Geometric spreading does not lose energy in this situation; the energy just spreads out as the wave front expands (Heasley, 2009). Geometric spreading differs for surface and body waves. For body waves, a spherical wave front is developed and the waves are moving away from the signal source equally in all directions as shown in Figure 3.3 . 


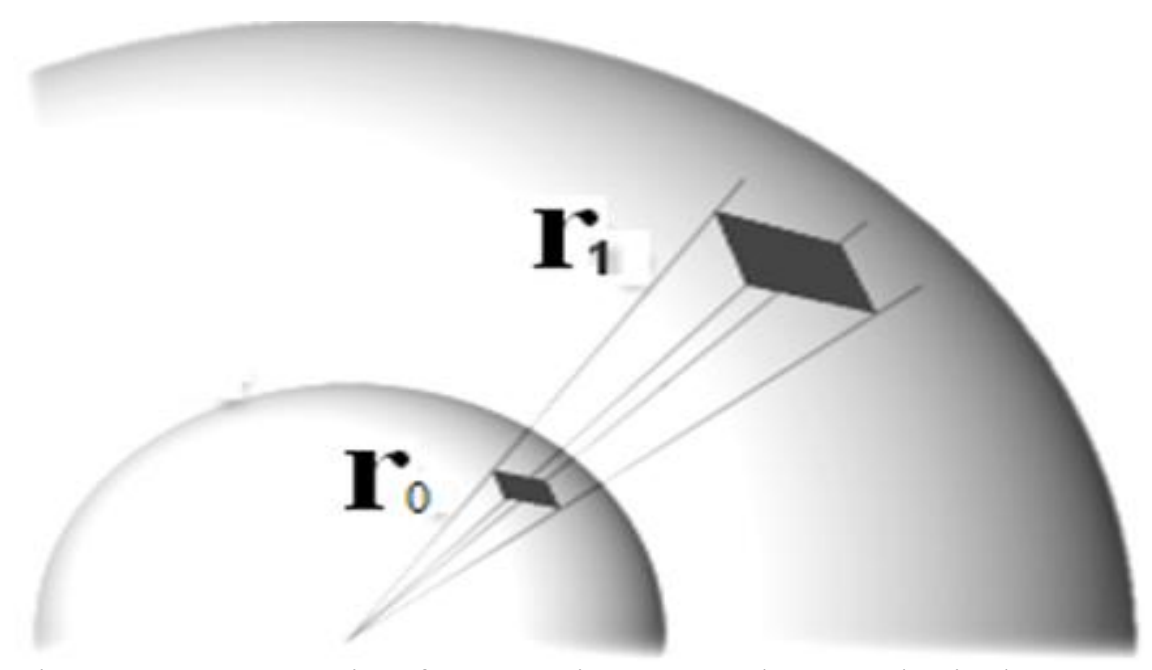

Figure 3.3. Attenuation from a point source due to spherical geometric spreading, (Radar Technology Tutorial Webpage).

We know that the surface area $\left(\mathrm{A}_{0}\right)$ of the inner shell is:

$$
\mathrm{A}_{0}=4 \pi \mathrm{r}_{0}^{2}
$$

and the surface area $\left(\mathrm{A}_{1}\right)$ of the outer shell is:

$$
\mathrm{A}_{1}=4 \pi \mathrm{r}_{1}^{2}
$$

where:

$$
\begin{aligned}
& r_{0} \quad=\text { radius of inner shell, } \\
& r_{1} \quad=\text { radius of outer shell, }
\end{aligned}
$$

Also, we know that the kinetic energy $\left(E_{k}\right)$ of the particle motion velocity which vibrate about an equilibrium point in the medium as,

$$
\mathrm{E}_{\mathrm{k}}=\frac{1}{2} \mathrm{~m}_{\mathrm{p}} \mathrm{v}_{\mathrm{p}}^{2}
$$

where:

$$
\begin{aligned}
& \mathrm{m}_{\mathrm{p}} \quad=\text { mass of the particle } \\
& \mathrm{v}_{\mathrm{p}} \quad=\text { particle velocity }
\end{aligned}
$$


and we know that the density $(\rho)$ is equal to the particle mass $\left(\mathrm{m}_{\mathrm{p}}\right)$ divided by the volume (Vol):

where:

$$
\rho=\frac{m_{p}}{\text { Vol }}
$$

$$
\begin{aligned}
& \rho \quad=\text { particle density } \\
& \text { Vol } \quad=\text { particle volume }
\end{aligned}
$$

therefore, if we look at the energy in a unit volume of material $(\mathrm{Vol}=1)$, then equation $(3.14)$ can be re-written as:

$$
\mathrm{E}_{\mathrm{k}}=\frac{1}{2} \rho \mathrm{v}_{\mathrm{p}}^{2}
$$

Equation (3.20) is known as kinetic energy density for a unit volume particle. If we ignore kinetic energy losses in the system, conservation of energy makes it clear that the total energy on the surface of the inner sphere, $A_{0}$, should be equal to the total energy on the surface of the outer sphere, $A_{1}$. This can be written as:

$$
\mathrm{E}_{\mathrm{k} 0} \times 4 \pi \mathrm{r}_{0}^{2}=\mathrm{E}_{\mathrm{k} 1} \times 4 \pi \mathrm{r}_{1}^{2}
$$

where:

$$
\begin{aligned}
& \mathrm{E}_{\mathrm{k} 0} \quad=\text { the energy density at } \mathrm{r}_{0} \\
& \mathrm{E}_{\mathrm{k} 1} \quad=\text { the energy density at } \mathrm{r}_{1}
\end{aligned}
$$

Simplifying and re-arranging we see that:

$$
\mathrm{E}_{\mathrm{k} 1}=\mathrm{E}_{\mathrm{k} 0} \frac{\mathrm{r}_{0}^{2}}{\mathrm{r}_{1}^{2}}
$$

and we can conclude that the energy per wave front unit volume decays as $1 / \mathrm{r}^{2}$ with distance from the energy source.

Knowing that the energy density per unit volume is a function of the material density as show in equation (3.20), we can substitute equation (3.20) into equation (3.22): 


$$
\frac{1}{2} \rho \mathrm{v}_{\mathrm{p} 1}^{2}=\frac{1}{2} \rho \mathrm{v}_{\mathrm{p} 0}^{2} \times \frac{\mathrm{r}_{0}^{2}}{\mathrm{r}_{1}^{2}}
$$

where:

$$
\begin{aligned}
& \mathrm{v}_{\mathrm{p} 0} \quad=\text { particle velocity at the inner shell } \\
& \mathrm{v}_{\mathrm{p} 1} \quad=\text { particle velocity at the outer shell }
\end{aligned}
$$

Simplifying and taking the square root of both sides we see that:

$$
\mathrm{v}_{\mathrm{p} 1}=\mathrm{v}_{\mathrm{p} 0} \times \frac{\mathrm{r}_{0}}{\mathrm{r}_{1}}
$$

And we can conclude that particle velocity of the wave (which is linearly proportional to the amplitude) decreases as $1 / \mathrm{r}$ with distance from the energy source.

3.2.3 Inter-bed Reflection/Transmission: As seismic waves travel through the overburden medium and encounter layer boundaries, the energy of the incident wave is partitioned at each boundary. Part of the energy is reflected back into the first medium while the remaining part is transmitted into the second medium as shown in Figure 3.4 (Kennet, 1983). The ratio of the transmitted signal energy depends on the acoustic impedance of both the incident and refraction layers and also the angles of direction of the incidence and refracted angle as below given:

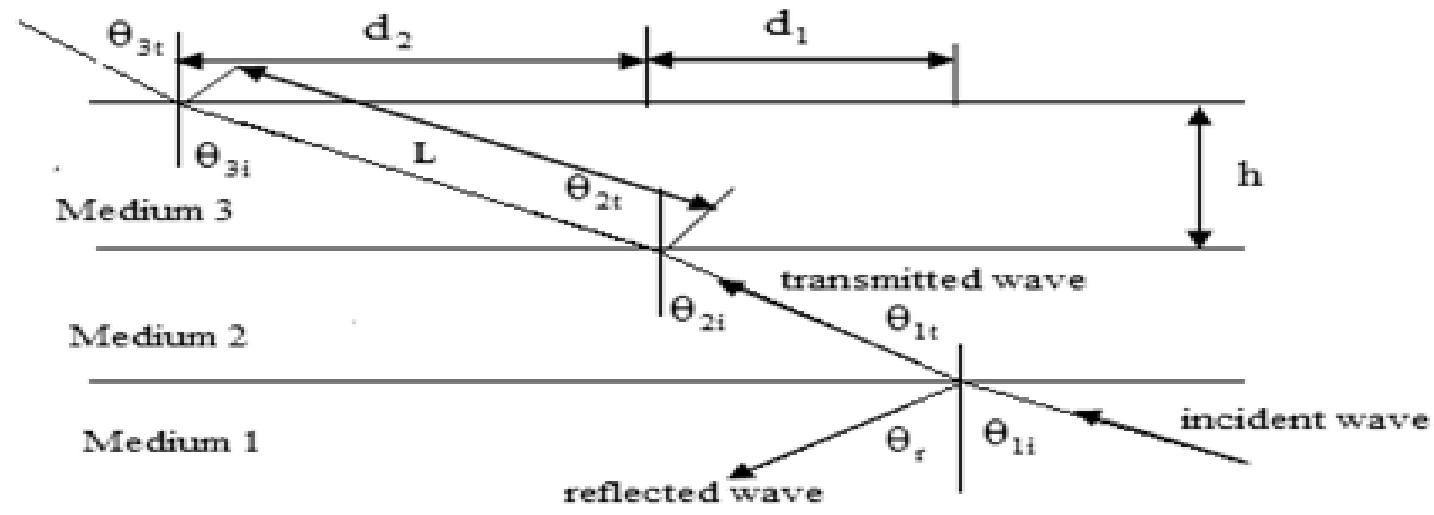

Figure 3.4. Signal reflection and transmission at the interface.

$$
\mathrm{T}=\frac{2 \mathrm{M}_{1} \operatorname{Cos} \theta_{\mathrm{i}}}{\mathrm{M}_{2} \operatorname{Cos} \theta_{\mathrm{i}}+\mathrm{M}_{1} \operatorname{Cos} \theta_{\mathrm{t}}}
$$


where:

$\mathrm{T}=$ Transmission coefficient of the interface between the incident and refraction layer

$\mathrm{M}_{1} \quad=$ Impedance of the incident layer

$\mathrm{M}_{2} \quad=$ Impedance of the refracted layer

$\theta_{\mathrm{i}} \quad=$ the angle of direction of the incidence angle

$\theta_{\mathrm{t}}=$ the angle of direction of the refracted angle

Acoustic impedance (M) is the product of the seismic velocity of the material and the density as given below:

$$
\mathrm{M}=\rho v
$$

where:

$\rho \quad=$ density of the layer

$\mathrm{v} \quad=$ seismic velocity in the layer

The angles of incidence and refraction at each layer can be determined by employing Snell's law.

Snell established a mathematical equation relating the angles of incident and angle refraction with the velocity of the two layers media on each side of the interface as shown below; 


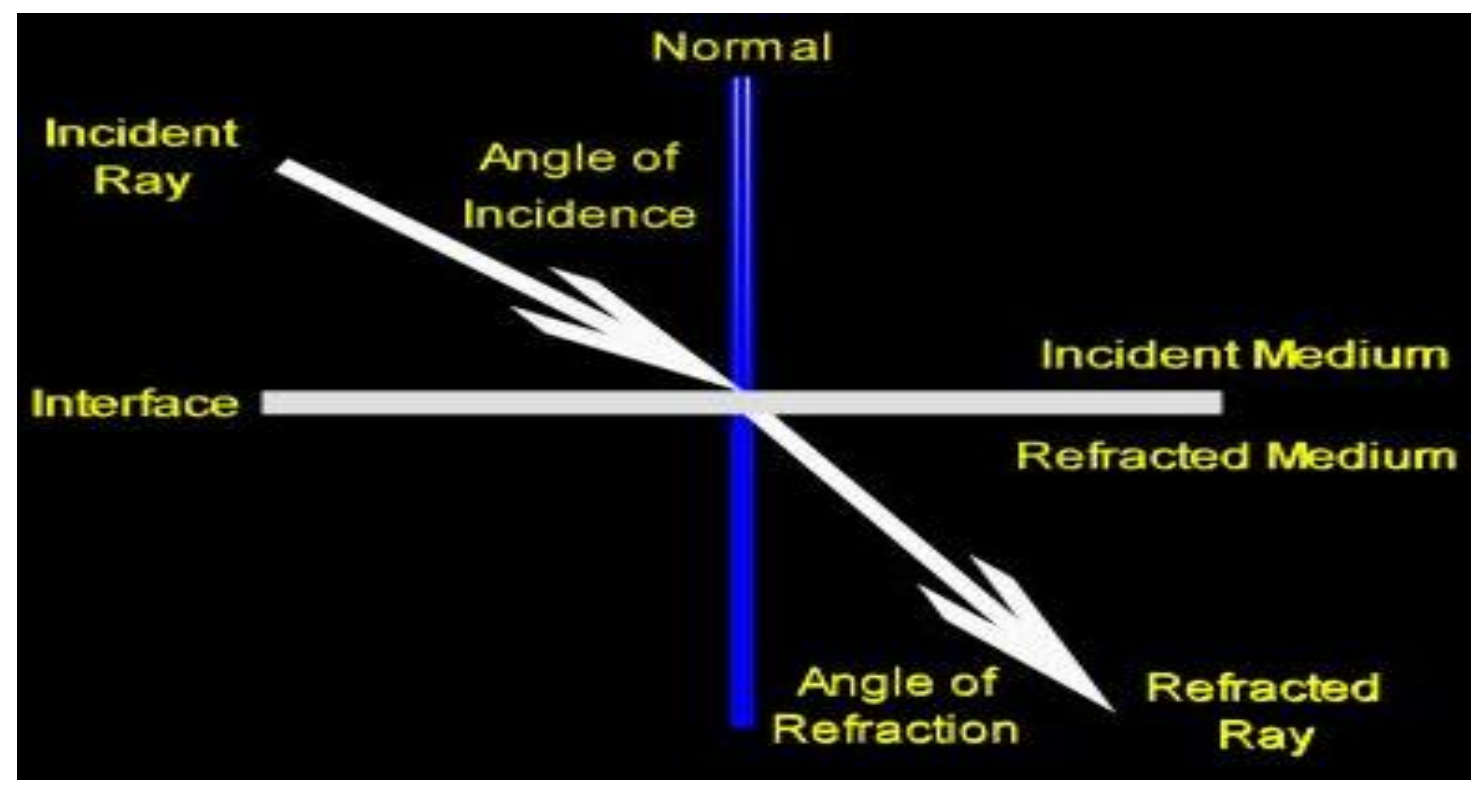

Figure 3.5. Angle of incidence and angle of refraction.

$$
\operatorname{Sin} \theta_{\mathrm{t}}=\frac{\mathrm{v}_{2}}{\mathrm{v}_{1}} \sin \theta_{\mathrm{i}}
$$

where:

$$
\begin{aligned}
& \mathrm{v}_{1}=\text { seismic velocity in the incident layer } \\
& \mathrm{v}_{2} \quad=\text { seismic velocity in the refracted layer }
\end{aligned}
$$

As shown in Figure 3.5, the incident wave, after striking the interface will either refract towards the normal or move away from the normal line based on the ratio of media velocities. If the velocity of the incident layer is lower than the velocity of the refracted layer, the incident wave will refract toward the normal line. Also, if the incident velocity is higher, the incident wave will move away from the normal line. This normal line is an imaginary line that is perpendicular to the interface at the point of refraction. The angle between the incident ray and the normal is called the incident angle while the angle between the refracted ray and the normal is known as the angle of refraction. 


\subsubsection{Summary of Signal Loss Mechanisms}

Signal attenuation into the medium occurs through various mechanisms. The three major sources which are described in this chapter are hysteresis, geometric spreading, and inter-bed reflection and transmission. While signal loss due to geometric spreading is distance dependent, signal loss due to hysteresis appear to be exponential with distance and also frequency dependent, (Geldart et al, $4^{\text {th }}$ edition). For the signal loss due to signal reflection, the physical properties of the propagating medium such as density and compressional velocity are the major factors that determine this type of signal loss. In the Table 3.2 below, the summary of these loss mechanisms and how it could be estimated are detailed.

Table 3.2 Summary of signal loss mechanisms.

\begin{tabular}{|l|l|l|}
\hline Signal Loss Mechanism & \multicolumn{1}{|c|}{ Comments } & Magnitude \\
\hline 1) Geometrical & $\begin{array}{l}\text { Signal amplitude depend on the } \\
\text { distance from the source, } \mathrm{r} \text { is the } \\
\text { radius of the expanding wave } \\
\text { fronts. } \\
\text { 2) Hysteresis }\end{array} \quad \begin{array}{l}\text { Loss of energy to heat, weighted } \\
\text { towards high frequency, the wave } \\
\text { energy attenuate exponentially } \\
\text { with distance, } \mathrm{r} \text {, where } \alpha \text { is the } \\
\text { coefficient of attenuation } \\
\text { Loss of energy due to waves } \\
\text { reflection/transmission and and } \\
\text { depend on the impedance of the } \\
\text { medium and the incident angle. }\end{array}$ & $\mathrm{T}=\frac{2 \rho_{2} \mathrm{v}_{2} \operatorname{Cos} \theta_{\mathrm{i}}}{\rho_{2} \mathrm{v}_{2} \operatorname{Cos} \theta_{\mathrm{i}}+\rho_{1} \mathrm{v}_{1} \operatorname{Cos} \theta_{\mathrm{t}}}$ \\
3) Reflecting layers & \multicolumn{1}{|c|}{} \\
\hline
\end{tabular}

\subsection{Particle velocity}

When the miner pounds on the roof, the particles in the medium transmit the seismic wave from the signal source to the surface geophone. The velocity of the oscillatory motion of these 
particles resulting in surface ground displacement is known as particle velocities. The peak particle velocity is usually used to determine the signal strength of the signaling miner at the surface. To ultimately determine the particle velocity at the surface from the miners pounding underground, the force-time function, $g(t)$, in equation (3.3) needs to be related to the displacement of the particles in the roof. White (1965) developed this relationship for the outgoing P-wave radial displacement, $\mathrm{d}_{\mathrm{r}}(\mathrm{t})$, for a point source in an infinite medium (see equation (3.28). The derivation of this equation is based on the theory that, for each unit of distance traveled by the seismic waves, the signal force function $\mathrm{g}(\mathrm{t})$ which represent the signal source attenuates (Durkin and Greenfield, 1981).

$$
d_{r}(t)=\frac{\cos \theta}{4 \pi \rho V^{2}} \times \frac{1}{R} \times g\left(t-\frac{R}{V}\right)
$$

where:

$\theta \quad=$ the angle between the source-receiver direction and the vertical

$\rho \quad=$ the density of the roof material

$\mathrm{R}=$ the radial distance between the source and the receiver

$\mathrm{V} \quad=$ the P-wave velocity of the medium.

In this equation, the geometric spreading loss is included with the $1 / \mathrm{R}$ term, and the (t-R/V) term offsets the time variable by the length of time it takes the signal to travel from the source to the receiver (distance divided by velocity), and the $\operatorname{Cos} \theta$ terms corrects for the angular difference between the transmitted wave and the vertical axis of the surface geophone..

If the energy loss due to dissipation in the media equation (3.15) and the energy loss due to reflection at changes in material equation (3.25) are included, then equation (3.28) can be written as: 


$$
d_{r}(t)=\frac{\cos \theta}{4 \pi \rho V^{2}} \times \frac{1}{R} \times e^{-\alpha R} \times T \times g\left(t-\frac{R}{V}\right)
$$

If the medium is layered, as for stratified sedimentary rock, then the total radial distance, $\mathrm{R}$, can be broken into the sum of the radial distance, $r_{n}$, through each layer of the $n$ layers:

$$
\mathrm{R}=\mathrm{r}_{1}+\mathrm{r}_{2}+\cdots+\mathrm{r}_{\mathrm{n}}
$$

Similarly, the attenuation factor becomes the sum of the attenuation in each layer:

$$
\mathrm{e}^{-\alpha \mathrm{R}} \Rightarrow \mathrm{e}^{-\sum_{1}^{\mathrm{n}} \alpha_{\mathrm{n}} \mathrm{r}_{\mathrm{n}}}
$$

And the transmission coefficient becomes:

$$
\mathrm{T}_{\mathrm{n}} \Rightarrow \frac{2 \rho_{2} \mathrm{v}_{2} \cos \theta_{\mathrm{i}}}{\rho_{1} \mathrm{v}_{1} \cos \theta_{\mathrm{i}}+\rho_{2} \mathrm{v}_{2} \cos \theta_{\mathrm{t}}} \times \frac{2 \rho_{3} \mathrm{v}_{3} \cos \theta_{\mathrm{i}}}{\rho_{2} \mathrm{v}_{2} \cos \theta_{\mathrm{i}}+\rho_{3} \mathrm{v}_{3} \cos \theta_{\mathrm{t}}} \times \frac{2 \rho_{\mathrm{n}} \mathrm{v}_{\mathrm{n}} \cos \theta_{\mathrm{i}}}{\rho_{\mathrm{n}-1} \mathrm{v}_{\mathrm{n}-1} \cos \theta_{\mathrm{i}}+\rho_{\mathrm{n}} \mathrm{v}_{\mathrm{n}} \cos \theta_{\mathrm{t}}}
$$

And the delay time until the seismic wave gets between the underground source and the surface receiver becomes the summation of the travel time through each layer

$$
\frac{R}{V} \Rightarrow \sum_{1}^{n} \frac{r_{n}}{v_{n}}
$$

Now, substituting equations (3.30) through, (3.33) back into equation (3.29), and expanding the function $\mathrm{g}(\mathrm{t})$ with equation (3.4), we get the radial displacement-time function for a remote point in a layered medium due to the miners pounding on the roof:

$$
\mathrm{d}_{\mathrm{r}}(\mathrm{t})=\frac{\cos \theta}{4 \pi \rho_{1} \mathrm{v}_{1}^{2}} \times \frac{1}{\mathrm{R}} \times \mathrm{e}^{-\sum_{1}^{\mathrm{n}} \alpha_{\mathrm{n}} \mathrm{r}_{\mathrm{n}}} \times \mathrm{T}_{\mathrm{n}} \times \mathrm{g}_{0} \sin \left(\frac{2 \pi}{\tau_{\mathrm{S}}}\left(\mathrm{t}-\sum_{1}^{\mathrm{n}} \frac{\mathrm{r}_{\mathrm{n}}}{\mathrm{v}_{\mathrm{n}}}\right)\right)
$$

Most seismic equipment records a velocity curve, so by differentiating the above equation with respect to time, we get the radial velocity-time function, $v_{r}(t)$, for a remote point in a layered medium due to the miners pounding on the roof: 


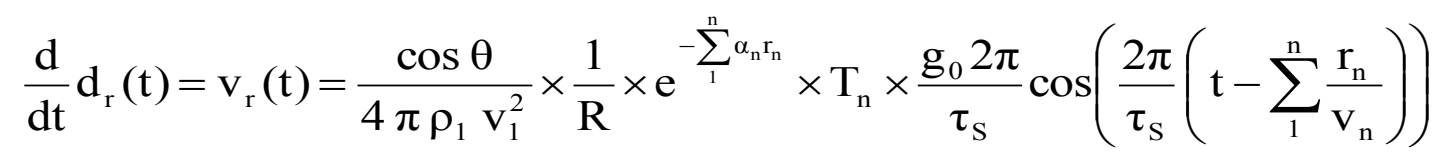




\section{CHAPTER FOUR}

\section{SEISMIC FIELD TESTS}

\subsection{Introduction}

Two different field tests were conducted at two underground coal mines around Morgantown, West Virginia. The first field test was conducted at 4-West Mine of Dana Mining and the second test was conducted at the Federal \#2 Mine of Peabody Energy. During the field tests, geophones were placed at the surface while an underground team created a series of pounding signals with different pounding devices and on different pounding surfaces. For each combination of pounding device and pounding surface, the effect of the mine geology, mine depth, and geophone offset distance were investigated.

\subsection{Field Test Study at the 4-West Mine of Dana Mining}

The 4-West Mine of Dana Mining is located a few miles from the Mt. Morris Exit (Exit \#1)

off of Interstate 79, just north of Morgantown, West Virginia and the Pennsylvania border (see Figure 4.0). 


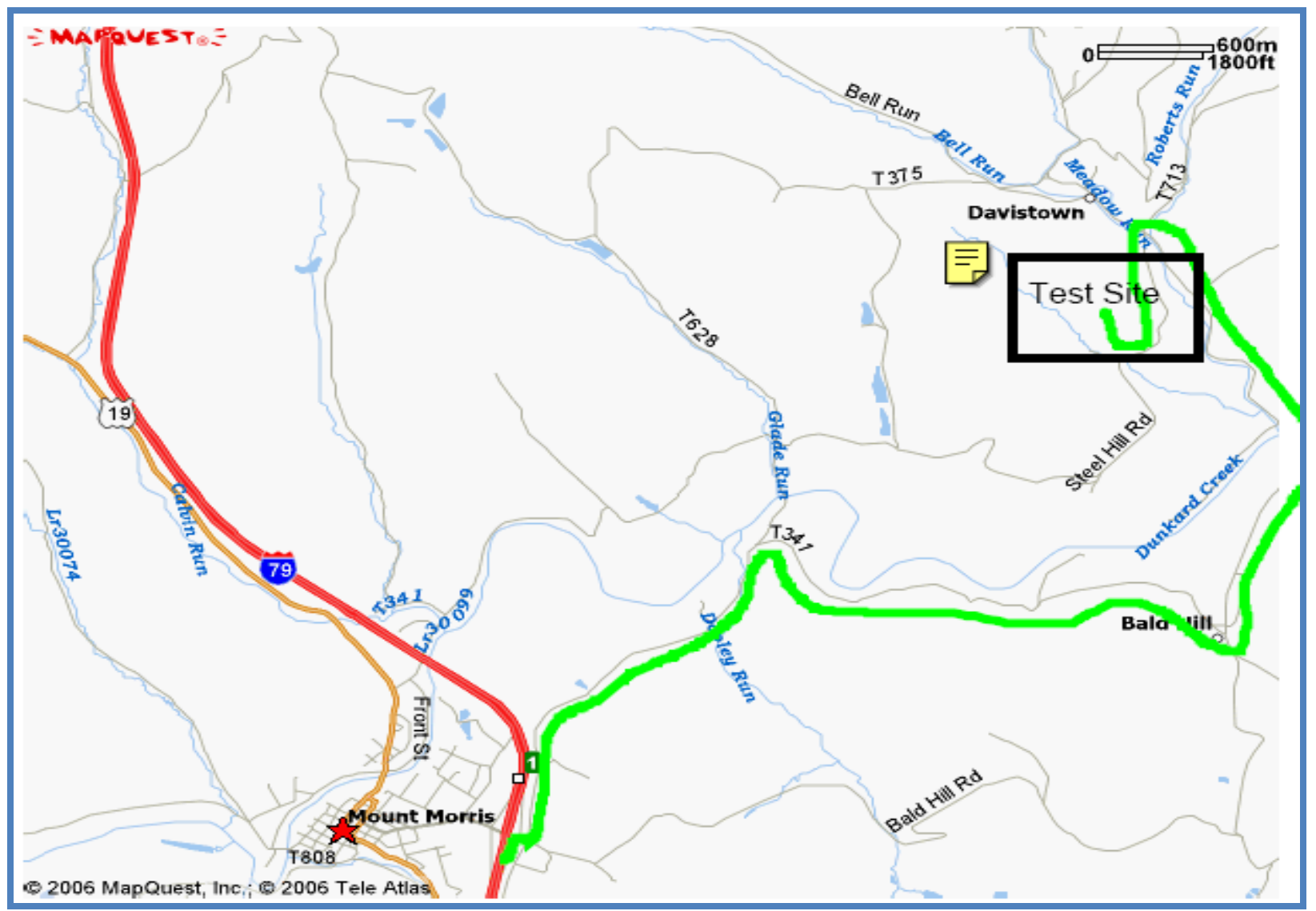

Figure 4.0. Location map of 4-West Mine of Dana Mining seismic test site, (Heasley, 2006).

The depth of cover at this mine was surveyed at $441 \mathrm{ft}$. At the time of the field test, 4-West Mine of Dana Mining was operating one continuous miner section which was driving an eleven entry main in a southwestern direction. The exact location for the test was chosen near the top of a local ridge in order to get the maximum amount of overburden and it was directly above the Haulage Entry in the mine for ease of underground access (see Figure 4.1). The surface cover at the site was a hay field which allowed very easy access for installing the seismic system. 


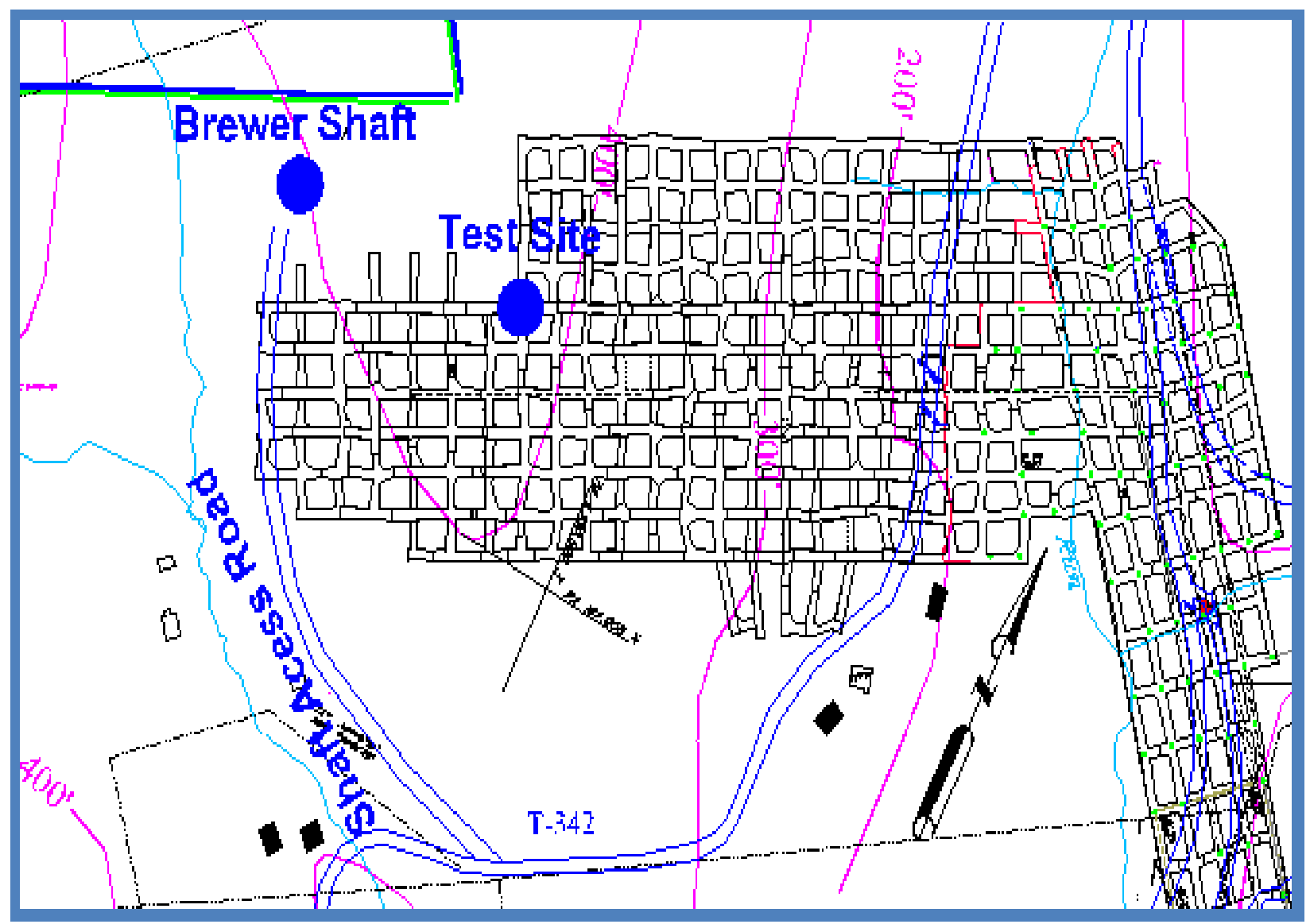

Figure 4.1. Location of the seismic test site, (Heasley, 2006).

\subsubsection{Geological Layers of 4-West Mine of Dana Mining}

In order to have an idea of the nature and thickness of each geological layer in the overburden of the field test site, the drilling log from a nearby mine shaft to the test area was employed, (see Table 4.1). Between this mine shaft and the test area, there is an elevation difference due to the topographic relief of the surface. Because of this elevation difference, the drilling log presented in Table 4.0 could not include the information (name and thickness) of the geological layers that are close to the surface. An approximate guess of the information about this area was made from past experience. 
Table 4.0. Geological layers of the 4-West Mine of Dana Mining.

\begin{tabular}{|c|c|c|c|}
\hline Depth to Bottom & Thickness & Rock Type & Rock Characteristics \\
\hline 10.0 & 10.00 & Subsoil & \\
\hline 40.0 & 30.00 & Sandstone & Light Gray, Hard \\
\hline 54.0 & 14.00 & Sandstone & Light Gray, Very Hard, Clayey, Fractured \\
\hline 62.0 & 8.00 & Sandstone & Light Gray, Hard \\
\hline 64.5 & 2.50 & Sandstone & Light Gray, Hard, Silty, Pyrite \\
\hline 70.0 & 5.50 & Shale & Tan, Medium-Hard \\
\hline 70.5 & 0.50 & Shale & Light Gray, Firm \\
\hline 78.5 & 8.00 & Waynesburg Coal & Black \\
\hline 86.0 & 7.50 & Shale & Light Gray, Hard, Silty, Pyrite \\
\hline 89.0 & 3.00 & Shale & Black, Soft, Shaley, Nods \\
\hline 95.5 & 6.50 & Shale & Light Gray, Hard, Limey, Laminated \\
\hline 103.5 & 8.00 & Shale & Light Gray, Medium-Hard, Silty, Pyrite \\
\hline 104.0 & 0.50 & Bone & White, Soft \\
\hline 118.5 & 14.50 & Limestone & Light Gray, Shaley, Nods \\
\hline 121.5 & 3.00 & Clay & Light Gray, Very Hard \\
\hline 123.5 & 2.00 & Siltstone/Limestone & Medium Gray, Soft, Clayey, Fractured \\
\hline 137.5 & 14.00 & Shale & Light Gray, Hard, Limey, Laminated \\
\hline 140.5 & 3.00 & Limey Bone & Tan, Soft \\
\hline 143.0 & 2.50 & Clay & Light Gray, Soft, Limey, Laminated \\
\hline 145.5 & 2.50 & Shale & White, Soft, Shaley, Nods \\
\hline 148.0 & 2.50 & Shale & Light Gray, Very Soft, Coaly, Poor Formation \\
\hline 148.5 & 0.50 & Shale & Medium Gray, Hard \\
\hline 160.5 & 12.00 & Limestone & Light Gray, Hard, Limey, Laminated \\
\hline 164.5 & 4.00 & Limestone/Shale & Dark Gray, Hard \\
\hline 168.5 & 4.00 & Siltstone & Medium Gray, Shaley, Nods \\
\hline 176.5 & 8.00 & Sandstone & Dark Gray, Medium-Hard, Limey, Laminated \\
\hline 192.5 & 16.00 & Limestone & Medium Gray, Hard \\
\hline 195.5 & 3.00 & Shale & Light Gray, Medium-Hard \\
\hline 208.5 & 13.00 & Shale & Light Gray, Hard, Limey, Laminated \\
\hline 216.0 & 7.50 & Sandstone & Green Gray, Medium-Hard \\
\hline 218.5 & 2.50 & Limestone/Shale & Green Gray, Hard \\
\hline 222.5 & 4.00 & Limestone & Dark Gray, Shaley, Nods \\
\hline 235.5 & 13.00 & Shale & Medium Gray, Medium-Hard, Shaley, \\
\hline 236.5 & 1.00 & Limestone & Medium Gray, Hard \\
\hline 238.0 & 1.50 & Shale & Green, Hard \\
\hline 238.5 & 0.50 & Cont & Medium Gray, Soft, Shaley, Nods \\
\hline 243.2 & 4.70 & Carb Shale & Dark Gray \\
\hline 244.7 & 1.50 & Limestone & Hard, Limey, Laminated \\
\hline 248.5 & 3.80 & Cont Fossil & Medium Gray, Hard, Shaley, Nods \\
\hline 250.5 & 2.00 & Shale & Light Gray \\
\hline 253.9 & 3.40 & Limestone & Medium-Hard, Clayey, Fractured \\
\hline
\end{tabular}




\begin{tabular}{|c|c|c|c|}
\hline 264.5 & 10.60 & Limestone & Green Gray, Hard, Shaley, Nods \\
\hline 266.7 & 2.20 & Shale & Medium Gray, Very Hard, Silty, Pyrite \\
\hline 268.5 & 1.80 & Cont & White, Hard \\
\hline 269.7 & 1.20 & Limestone Nods & Medium-Hard, Limey, Laminated \\
\hline 271.3 & 1.60 & Limestone & Medium Gray \\
\hline 278.5 & 7.20 & Limestone & Dark Gray, Medium-Hard, Shaley, Nods \\
\hline 280.5 & 2.00 & Cont & Light Gray, Very Hard \\
\hline 282.5 & 2.00 & Shale & Light Gray, Soft, Shaley, Nods \\
\hline 284.2 & 1.70 & Limestone & Green Gray, Hard, Shaley, Nods \\
\hline 288.5 & 4.30 & Shale & Dark Gray, Hard \\
\hline 291.5 & 3.00 & Shale & Dark Gray, Hard \\
\hline 293.5 & 2.00 & Siltstone & Dark Gray \\
\hline 295.2 & 1.70 & Sandstone & Medium-Hard \\
\hline 298.5 & 3.30 & Shale & Light Gray, Medium-Hard, Shaley, Nods \\
\hline 300.7 & 2.20 & Cont & Light Gray, Medium-Hard, Shaley, Nods \\
\hline 308.5 & 7.80 & Sandstone & Sewickley Coal \\
\hline 311.5 & 3.00 & & \\
\hline & & & \\
\hline
\end{tabular}

\subsection{Seismic System Installation at the 4-West Mine of Dana Mining}

For the field test, four surface geophones and two tri-axial downhole geophones were placed in a "T" pattern (see Figure 4.2) on the surface.

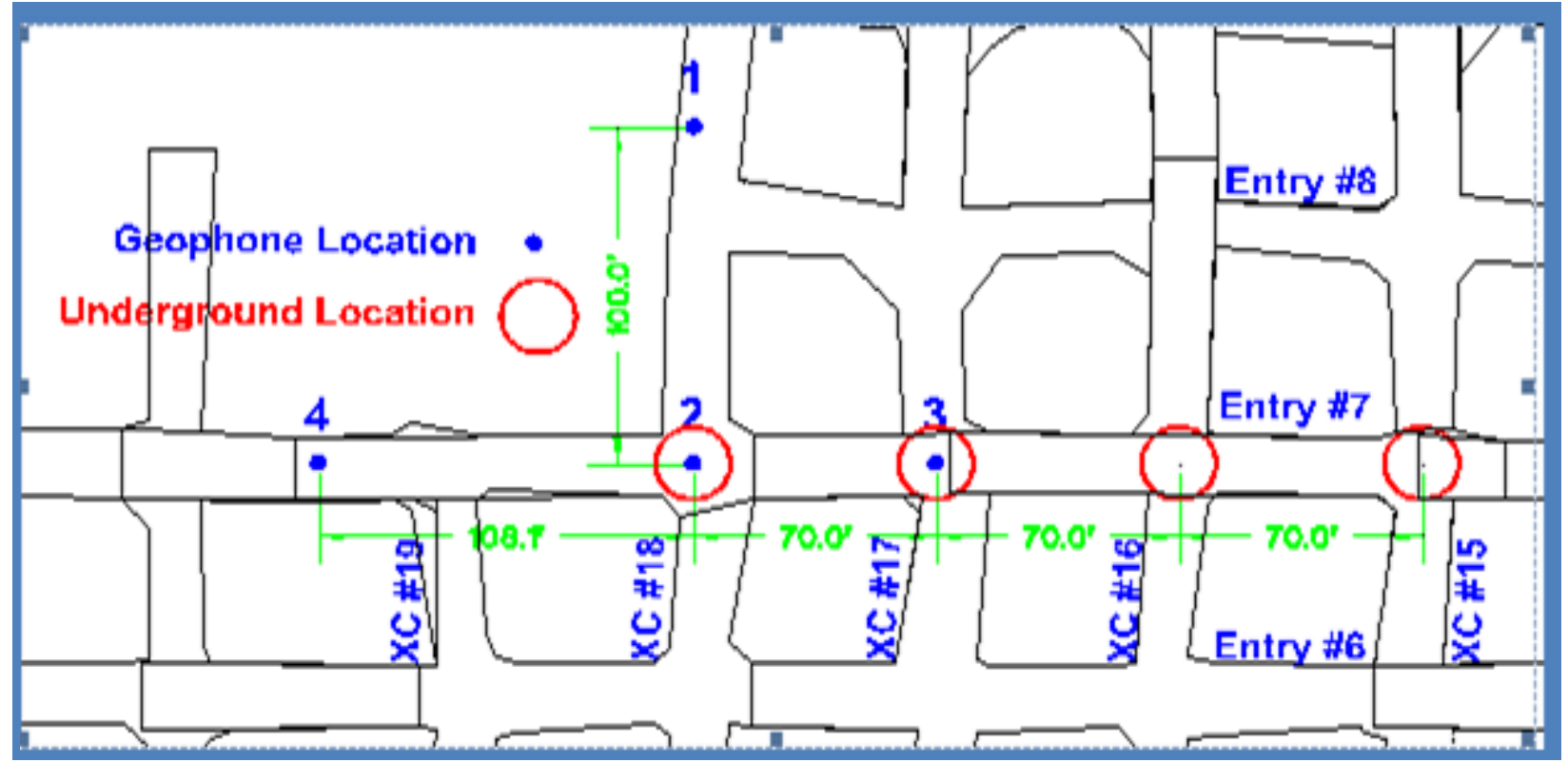

Figure 4.2. Location of the surface geophones and underground pounding sites, (Heasley, 2006). 
The four surface geophones were simply buried under one shovel full of the soil and were oriented with their " $y$ " axis pointing in an inby direction. These four surface geophones (\#1-\#4) were installed at locations 1-4, respectively, as shown in Figure 4.2. Geophone location 2 is the center of the " $T$ " and is located directly over entry \#7 and crosscut \#18. The depth of cover at Location \#2 was surveyed at $441 \mathrm{ft}$. The two down-hole geophones (\#5 and \#6) were placed vertically in shallow ( $3 \mathrm{ft}$ deep) auger holes. These down-hole geophones were wedged into the hole with a crowbar and then buried with soil that was lightly tapped. Geophone \#5 was installed at location 1 while Geophone \#6 was installed at location 2, which is also at the center of the "T". For the underground signaling, the first pounding was created at entry \#7 and crosscut \#18 directly below geophone location 2. Subsequent underground signaling was performed at: entry $\# 7$, crosscut \#17 (under geophone location 3); entry \#7, crosscut \#16; and entry \#7, crosscut \#15 (see Table 4.1).

Table 4.1. Location of the geophones and underground signaling sites.

\begin{tabular}{|c|l|l|l|c|l|l|}
\hline $\begin{array}{c}\text { Survey } \\
\text { Point }\end{array}$ & Coordinates & $\begin{array}{l}\text { Surface } \\
\text { Elevation }\end{array}$ & Geophone Location & $\begin{array}{l}\text { Mine Signal } \\
\text { Location }\end{array}$ & $\begin{array}{l}\text { Mine } \\
\text { Elevation }\end{array}$ \\
\hline T1 & 25192.52 & 41065.72 & 1186.29 & $\# 3$ & Entry \#7, XC17 & \\
\hline T2 & 25131.07 & 41032.20 & 1202.13 & $\# 2, \# 6(\# 6$ is buried) & Entry \#7, XC18 & 760.74 \\
\hline T3 & 25036.23 & 40980.39 & 1206.51 & $\# 4$ & & \\
\hline T4 & 25083.37 & 41120.04 & 1202.00 & $\# 1, \# 5(\# 5$ is buried) & & \\
\hline & 25253.94 & 41099.31 & & & Entry \#7, XC16 & \\
\hline & 25315.37 & 41132.86 & & & Entry \#7, XC15 & \\
\hline
\end{tabular}

\subsection{Seismic Equipments used at 4-West Mine of Dana Mining Field Study}

The seismic equipments used for the field test include the following:

1) Terrascience Microseismic Acquisition

2) Geophones

3) Signaling devices 
4.3.1 Terrascience Microseismic Equipment: The Terrascience Microseismic Acquisition unit (TMA) was a major component used in data acquisition and recording (see Figure 4.3). This unit collects and digitizes data from the sensors and sends the raw data to the TAR, Terrascience Acquisition Recorder. TAR consists of specially designed software that helps to store the data which signals an event into the database. The standard configuration of the TMA consists of 4 to 24 channels of 24-bit A/D modules in 4-channel increments, (Heasley, 2006). For the field test, 18 channels were used and the data was sampled at 2000 samples per second. Each TMA unit contained its own GPS module. This GPS was used to time stamp each data reading with precision. The data from TMA were sent to a portable PC at the site to be viewed, analyzed, and stored.

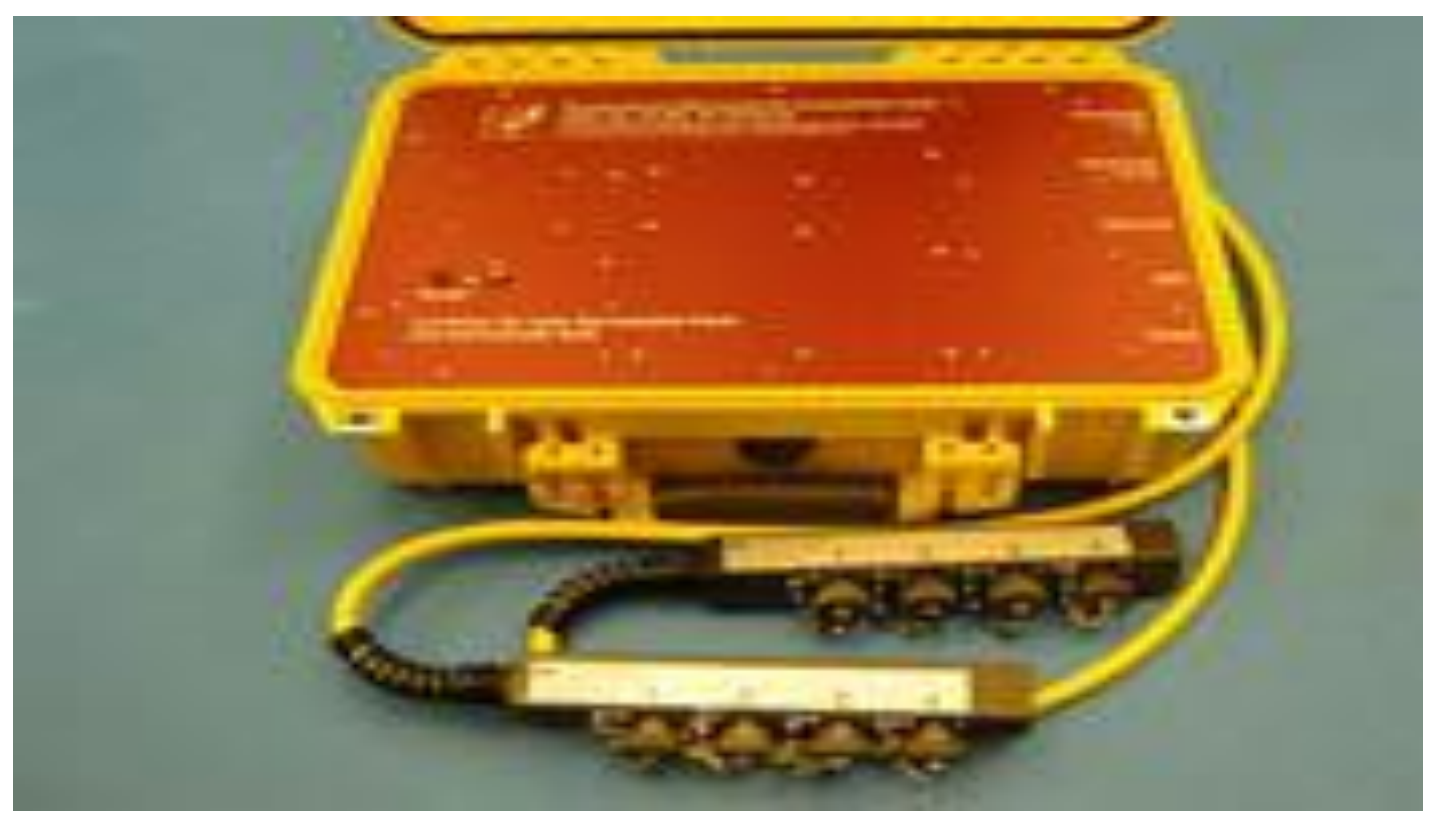

Figure 4.3. Terrascience's micro seismic acquisition equipment, (Weir-Jones Group Products Webpage).

\subsubsection{Geophones}

The geophones used for the study were Geospace 32CT elements. These geophones have a spectral band range from $\sim 10 \mathrm{~Hz}$ to $>500 \mathrm{~Hz}(3 \mathrm{~dB}$ down at $\sim 7 \mathrm{~Hz})$. The surface packages had one sensor along each orthogonal axis, three sensors in all. The downhole sensor packages had 
five tri-axial orthogonal arrays. Thus, there were five sensors along the $\mathrm{X}$-axis, five sensors along the Y-axis, and five sensors along the Z-axis, (Heasley, 2006) (Details on geophone are included in Chapter 2). The individual readings from each of the axis were added up to help cancel out the noise and improve the response of the sensor package to the ground vibration.

4.3.3 Signaling Devices: During the field test, a number of different signaling devices were used. These signaling devices were those considered to be readily available, very simple to use, and could be used within the safety limits of the underground mine. The signaling devices, which were employed, include a sledgehammer and a crib block. Besides these two devices that require pounding, three powder actuated tools(a Hilti DX76, a Hilti DX460, and a Hilti DX462) were also employed to generate signals (see Figure 4.4). These tools use a powder charge to drive the head into the coal face. The impact of the head with the rock face provides the seismic wave.

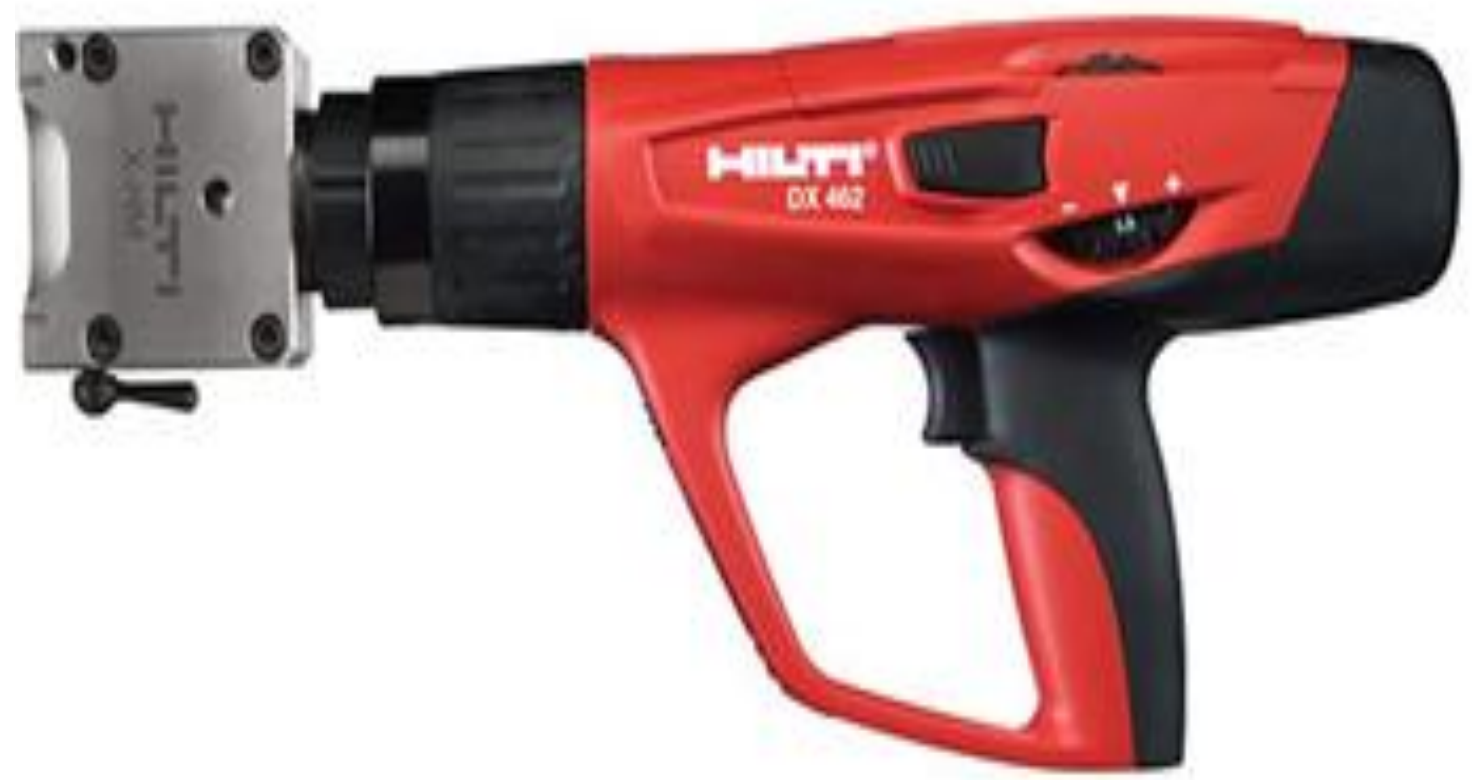

Figure 4.4. Hilti mining tool, (Hilti Corporation Products Webpage).

\subsection{Test Protocol for the 4-West Mine of Dana Mining Field Study}

During the field studies, an underground team created a series of pounding signals with pounding devices at different locations. For locations, the Hilti tools and pounding were 
performed directly on the roof, on a roof bolt, and on the coal rib. For each combination of signaling device and location, 5 hits were performed with a pause of 30 seconds occurred between each hit. During the signaling, the exact time that the pounding for each device had started was recorded. After a complete cycle, the underground team moved to the next location. The underground activity log is as shown in the Table 4.2 below.

Table 4.2. Seismic testing at 4-West Mine of Dana Mining, underground activity log.

\begin{tabular}{|c|c|c|}
\hline Starting Time & Activity & No. \\
\hline \multicolumn{3}{|c|}{ At Entry \#7, Crosscut \#18 } \\
\hline 9:00:00 & Hilti DX460 on Roof Rock & 5 \\
\hline 9:01:30 & Hilti DX76 on Roof Rock & 5 \\
\hline 9:03:00 & Hilti DX 462 on Roof Bolt Plate & 5 \\
\hline 9:04:05 & Hilti DX76 on Roof Bolt Plate & 5 \\
\hline 9:05:05 & Hammer on Roof Rock & 5 \\
\hline 9:06:00 & Hammer on Roof Bolt & 5 \\
\hline 9:06:30 & Crib Block on Roof Rock & 5 \\
\hline 9:07:00 & Crib Block on Roof Bolt & 5 \\
\hline 9:09:45 & Hilti DX460 on Rib & 5 \\
\hline $9: 10: 10$ & Hammer on Rib & 5 \\
\hline 9:10:50 & Crib Block on Rib & 5 \\
\hline \multicolumn{3}{|c|}{ At Entry \#7, Crosscut \#17 } \\
\hline 9:20:20 & Hilti DX460 on Roof Rock & 5 \\
\hline $9: 22: 30$ & Hilti DX76 on Roof Rock & 3 \\
\hline $9: 23: 28$ & Hilti DX76 on Roof Rock & 5 \\
\hline
\end{tabular}




\begin{tabular}{|c|c|c|}
\hline $9: 25: 55$ & Hilti DX 462 on Roof Bolt Plate & 5 \\
\hline 9:27:20 & Hilti DX76 on Roof Bolt Plate & 5 \\
\hline $9: 30: 00$ & Hammer on Roof Rock & 5 \\
\hline $9: 30: 30$ & Hammer on Roof Bolt & 5 \\
\hline $9: 31: 20$ & Crib Block on Roof Rock & 5 \\
\hline $9: 31: 40$ & Crib Block on Roof Bolt & 5 \\
\hline $9: 32: 15$ & Hilti DX460 on Rib & 5 \\
\hline $9: 33: 15$ & Hammer on Rib & 5 \\
\hline 9:33:55 & Crib Block on Rib & 5 \\
\hline \multicolumn{3}{|c|}{ At Entry \#7, Crosscut \#16 } \\
\hline 9:41:30 & Hilti DX460 on Roof Rock & 5 \\
\hline 9:42:40 & Hilti DX76 on Roof Rock & 5 \\
\hline 9:43:40 & Hilti DX 462 on Roof Bolt Plate & 5 \\
\hline 9:44:40 & Hilti DX76 on Roof Bolt Plate & 5 \\
\hline 9:45:40 & Hammer on Roof Rock & 5 \\
\hline 9:46:05 & Hammer on Roof Bolt & 5 \\
\hline 9:46:35 & Crib Block on Roof Rock & 5 \\
\hline 9:47:00 & Crib Block on Roof Bolt & 5 \\
\hline 9:46:10 & Hilti DX460 on Rib & 5 \\
\hline 9:48:55 & Hammer on Rib & 5 \\
\hline 9:49:50 & Crib Block on Rib & 5 \\
\hline \multicolumn{3}{|c|}{ At Entry \#7, Crosscut \#16 (Repeat) } \\
\hline 10:00:00 & Hilti DX460 on Roof Rock & 5 \\
\hline 10:01:03 & Hilti DX76 on Roof Rock & 5 \\
\hline
\end{tabular}




\begin{tabular}{|c|c|c|}
\hline $10: 02: 40$ & Hilti DX 462 on Roof Bolt Plate & 5 \\
\hline $10: 03: 20$ & Hammer on Roof Rock & 5 \\
\hline $10: 03: 45$ & Hammer on Roof Bolt & 5 \\
\hline 10:05:00 & Crib Block on Roof Rock & 5 \\
\hline $10: 05: 35$ & Crib Block on Roof Bolt & 5 \\
\hline 10:06:00 & Hilti DX76 on Roof Bolt Plate & 5 \\
\hline 10:07:30 & Hilti DX460 on Rib & 5 \\
\hline 10:08:00 & Hammer on Rib & 5 \\
\hline $10: 08: 35$ & Crib Block on Rib & 5 \\
\hline \multicolumn{3}{|c|}{ At Entry \#7, Crosscut \#15 } \\
\hline 10:30:00 & Hilti DX460 on Roof Rock & 5 \\
\hline $10: 30: 50$ & Hilti DX76 on Roof Rock & 5 \\
\hline 10:32:00 & Hilti DX 462 on Roof Bolt Plate & 5 \\
\hline $10: 32: 45$ & Hilti DX76 on Roof Bolt Plate & 5 \\
\hline $10: 33: 42$ & Hammer on Roof Rock & 5 \\
\hline 10:34:00 & Hammer on Roof Bolt & 5 \\
\hline $10: 35: 15$ & Crib Block on Roof Rock & 5 \\
\hline $10: 35: 40$ & Crib Block on Roof Bolt & 5 \\
\hline $10: 35: 55$ & Hilti DX460 on Rib & 5 \\
\hline $10: 36: 17$ & Hammer on Rib & 5 \\
\hline $10: 36: 44$ & Crib Block on Rib & 5 \\
\hline
\end{tabular}




\subsection{Results of the Seismic Field Study}

Upon quickly reviewing the results directly after the test, we were able to see a good strong response from 9 different underground signals set (see Table 4.2). From a detailed reviewing of the seismic data later, we found that:

Table 4.3 Seismic results of the buried geophone at "T" location on the surface.

\begin{tabular}{|c|c|c|c|c|}
\hline Time & Signal & $\begin{array}{l}\text { Peak Particle } \\
\text { Velocity }(\mathrm{m} / \mathrm{s})\end{array}$ & $\begin{array}{l}\text { Source Location } \\
\text { (441' deep) }\end{array}$ & $\begin{array}{c}\text { Horiz. } \\
\text { Offset } \\
\text { (ft) }\end{array}$ \\
\hline $9: 05: 00$ & Sledgehammer on Roof Rock & 0.000060 & Entry \#7, XC \#18 & 0 \\
\hline $9: 05: 34$ & Sledgehammer on Roof Bolt & 0.000060 & Entry \#7, XC \#18 & 0 \\
\hline $9: 06: 12$ & Crib Block on Roof Rock & 0.000100 & Entry \#7, XC \#18 & 0 \\
\hline $9: 06: 42$ & Crib Block on Roof Bolt & 0.000080 & Entry \#7, XC \#18 & 0 \\
\hline $9: 30: 08$ & Sledgehammer on Roof Rock & 0.000045 & Entry \#7, XC \#17 & 70 \\
\hline $9: 30: 42$ & Crib Block on Roof Rock & 0.000080 & Entry \#7, XC \#17 & 70 \\
\hline $9: 31: 10$ & Crib Block on Roof Bolt & 0.000080 & Entry \#7, XC \#17 & 70 \\
\hline $10: 03: 36$ & Sledgehammer on Roof Rock & 0.000025 & Entry \#7, XC \#16 & 140 \\
\hline $10: 04: 07$ & Crib Block on Roof Rock & 0.000045 & Entry \#7, XC \#16 & 140 \\
\hline
\end{tabular}

1) The crib block on the rock appeared to be the strongest signal followed by the crib block on the bolt, the sledgehammer on the roof rock, and the sledgehammer on the roof bolt. (See Table 4.2). Also, between the crib block and the sledgehammer, the crib block appeared to generate higher signal strength as plotted in Figure 4.5. 


\section{Comparison between Crib and Sledgehammer}

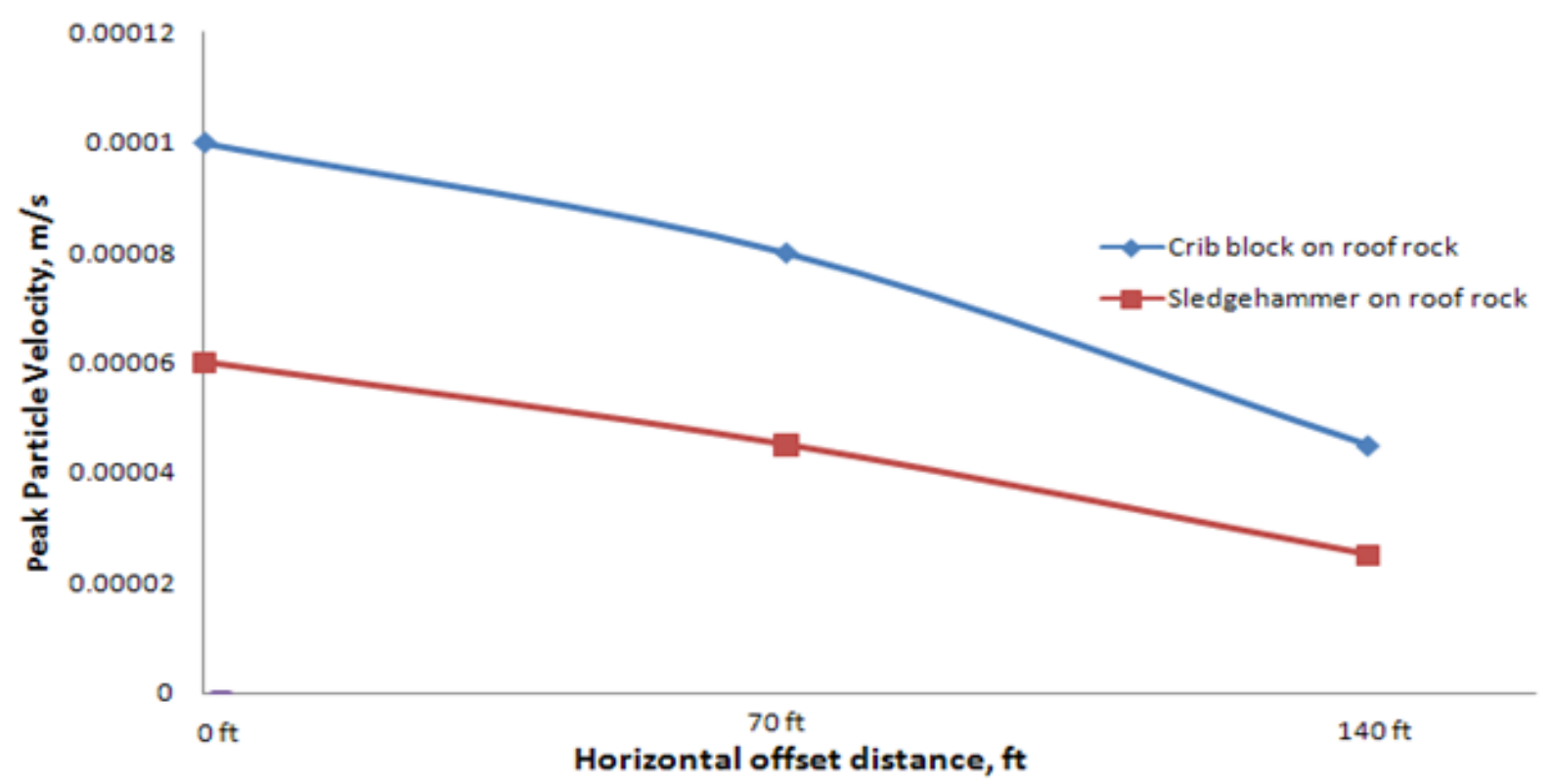

Figure 4.5. Comparison between seismic signal generated with a crib block and sledgehammer.

2) We had very good detectable signals at horizontal offsets of 0, 70 and 140, (see Figure 4.6). At $210 \mathrm{ft}$ of horizontal offset, maybe there was signal detection, but without knowing the time of the signal, it would be hard to pull the signal from background noise.

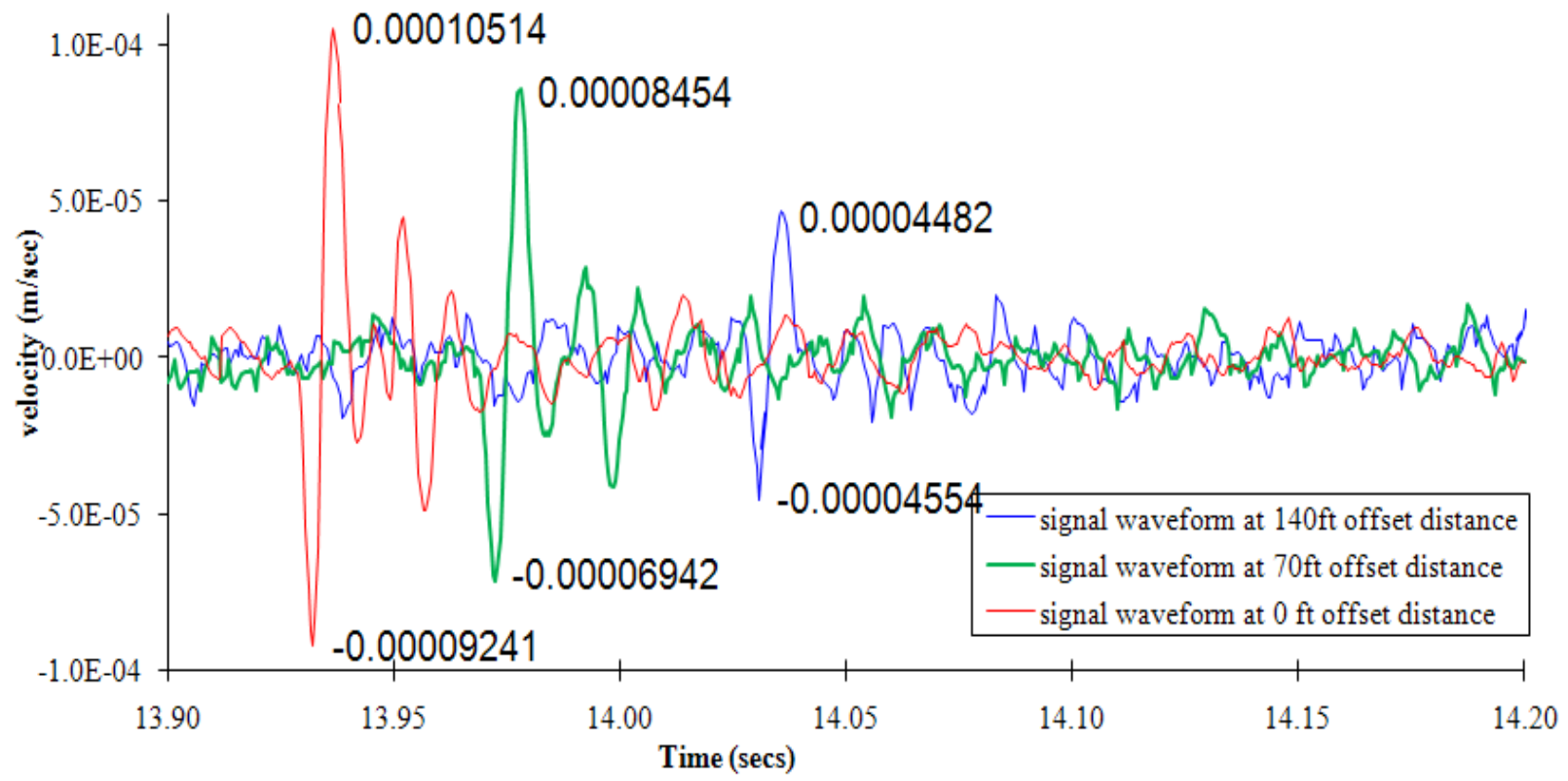

Figure 4.6. Comparison plot between signal from offset distance: $0 \mathrm{ft}, 70 \mathrm{ft}$ and $140 \mathrm{ft}$. 
3) We detected the strongest signals from the 0 and $70 \mathrm{ft}$ offset with both buried and surface geophones. The buried geophones provided more than twice the peak particle velocity, (see Figure 4.7).

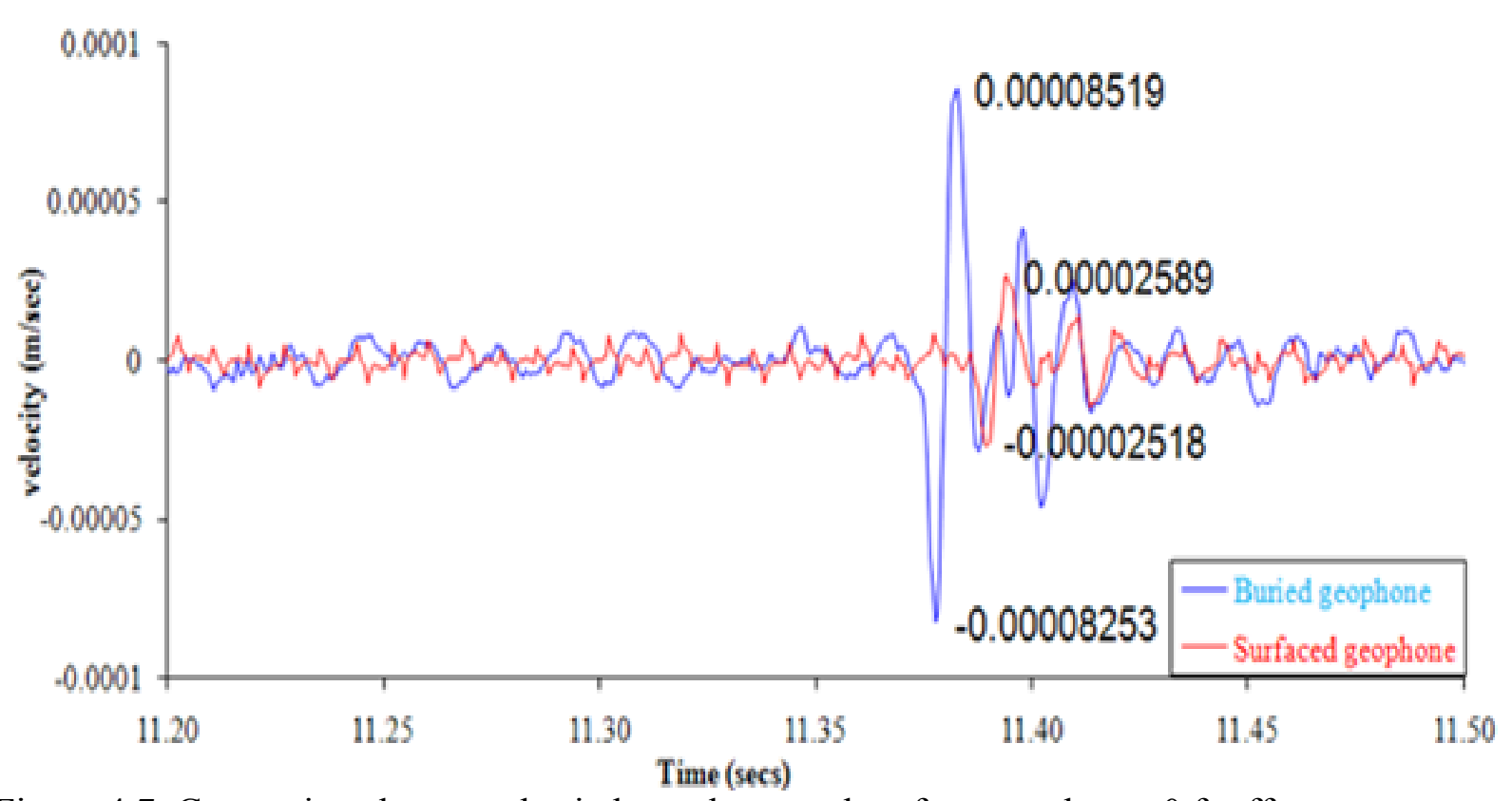

Figure 4.7. Comparison between buried geophone and surface geophone: $0 \mathrm{ft}$ offset.

4) In analyzing the seismic magnitude, it appears that just the increase in distance between the source and signal is not responsible for all of the signal attenuation that we see. We hypothesize that the incident angle between the "polarized" source and the sedimentary layers may be causing refraction/reflection (or some other mechanism) and greatly attenuating the non-perpendicular seismic signal.

The Hilti tools were only barely detectable when they were directly underneath the geophone, although they were obviously the loudest underground. We suspect that they may generate higher frequency signals, which attenuate fast. This is an area to investigate further. 


\subsection{Second Field Test at Federal \#2 Mine of Peabody Energy}

The second field test was conducted on Saturday, August $06^{\text {th }}, 2007$. The site location was at the Federal \#2 Mine located on Miracle Run Road, Fairview, West Virginia (see Figure 4.8-4.9). At this site, there was a pond by the hillside very close to the Federal \#2 Mine operating plant (see Figure 4.10). The overburden thickness at this site was $779 \mathrm{ft}$ (see Figure 4.11).

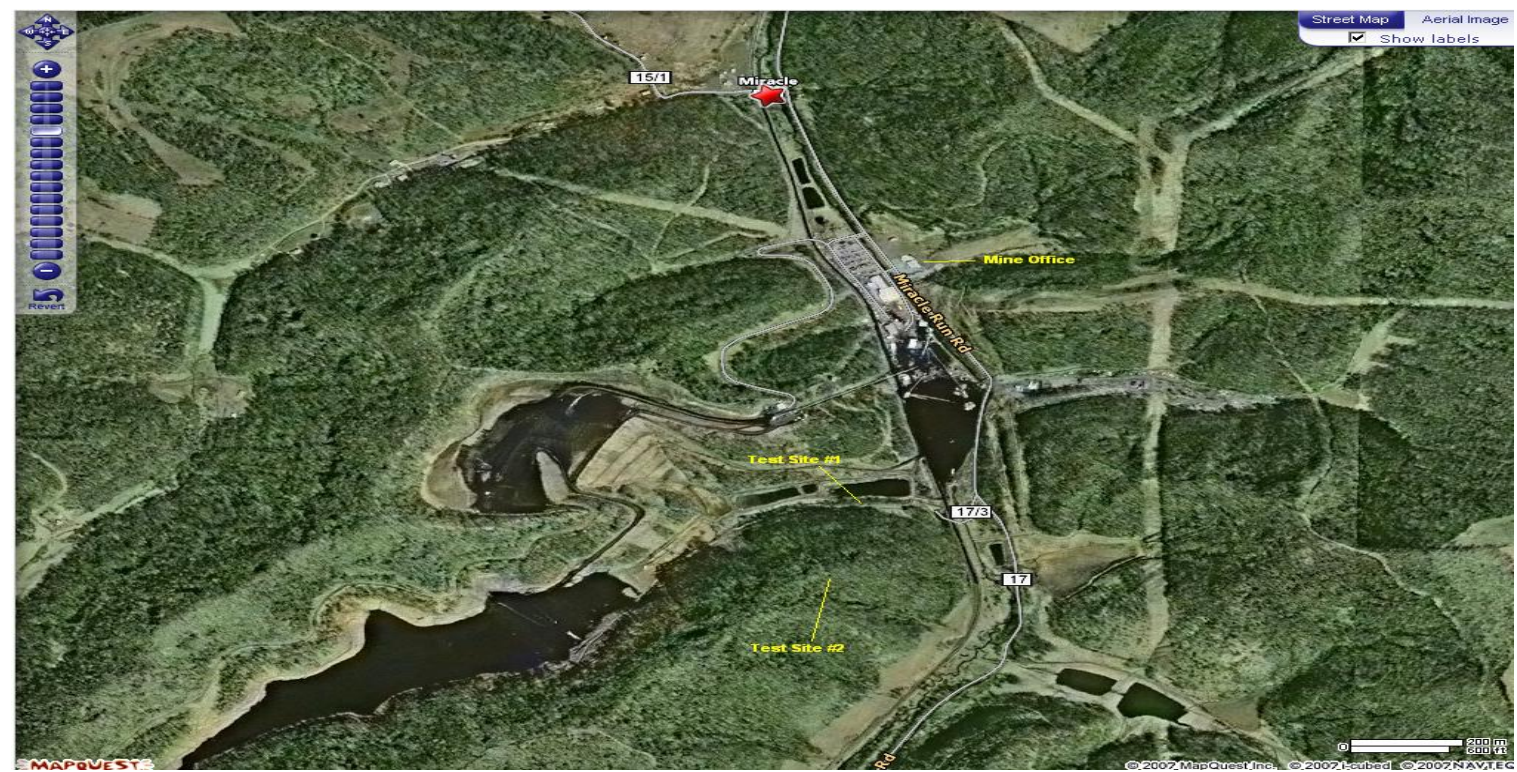

Figure 4.8. Google map showing the site location, (Heasley, 2006).

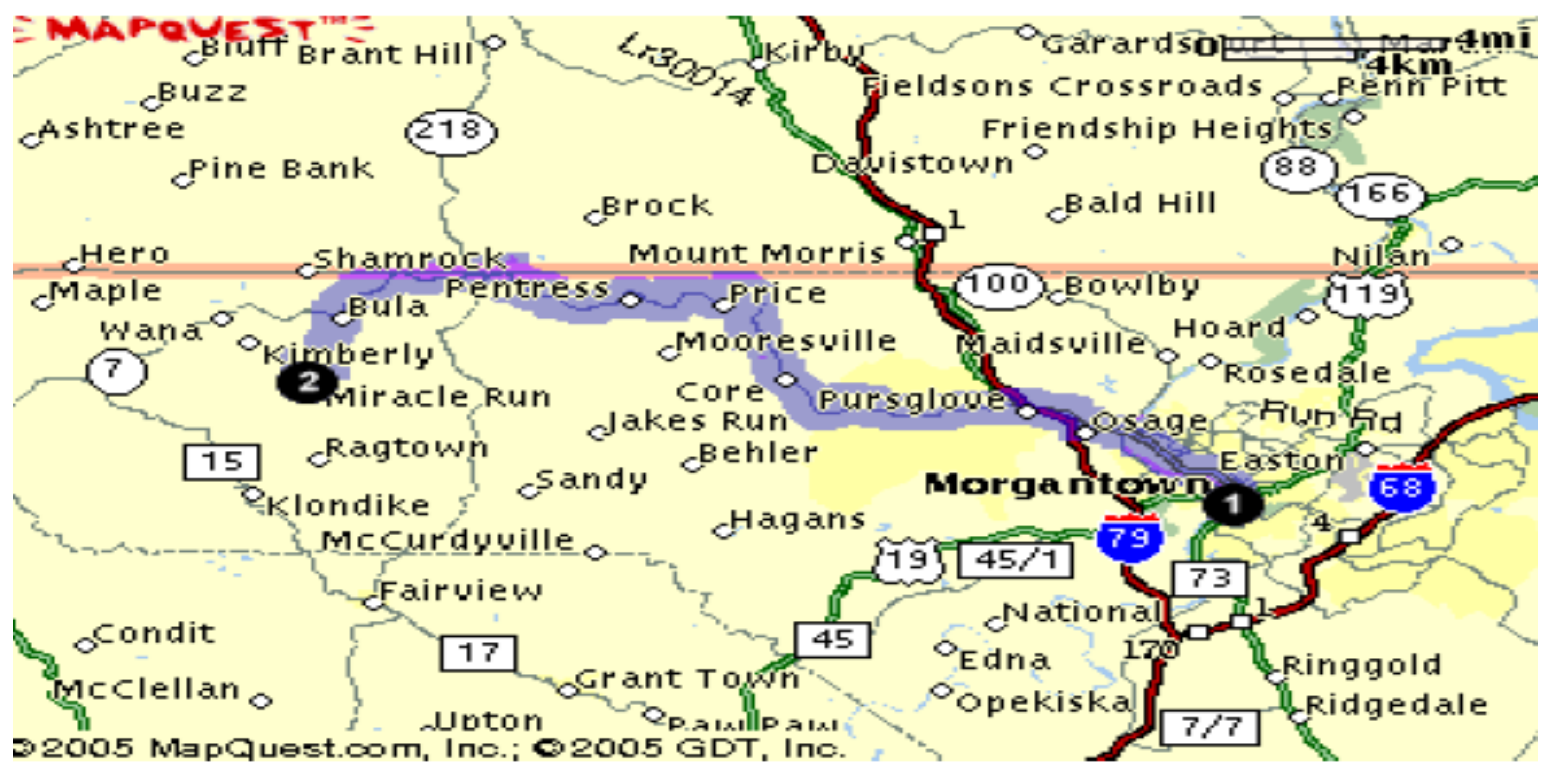

Figure 4.9. Map quest showing location of the field site, (Heasley, 2006). 


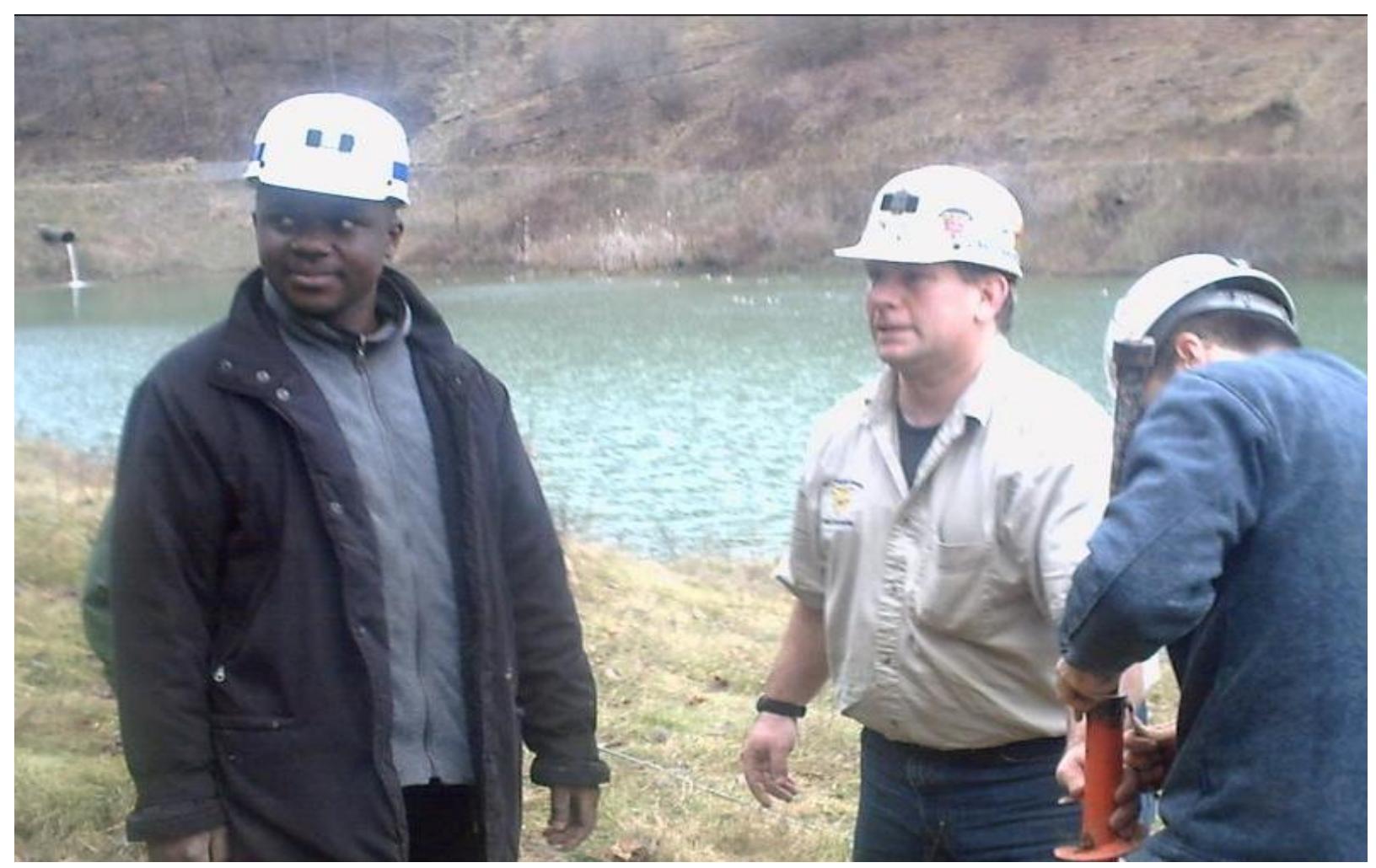

Figure 4.10. Some of the seismic team members by the Pond.

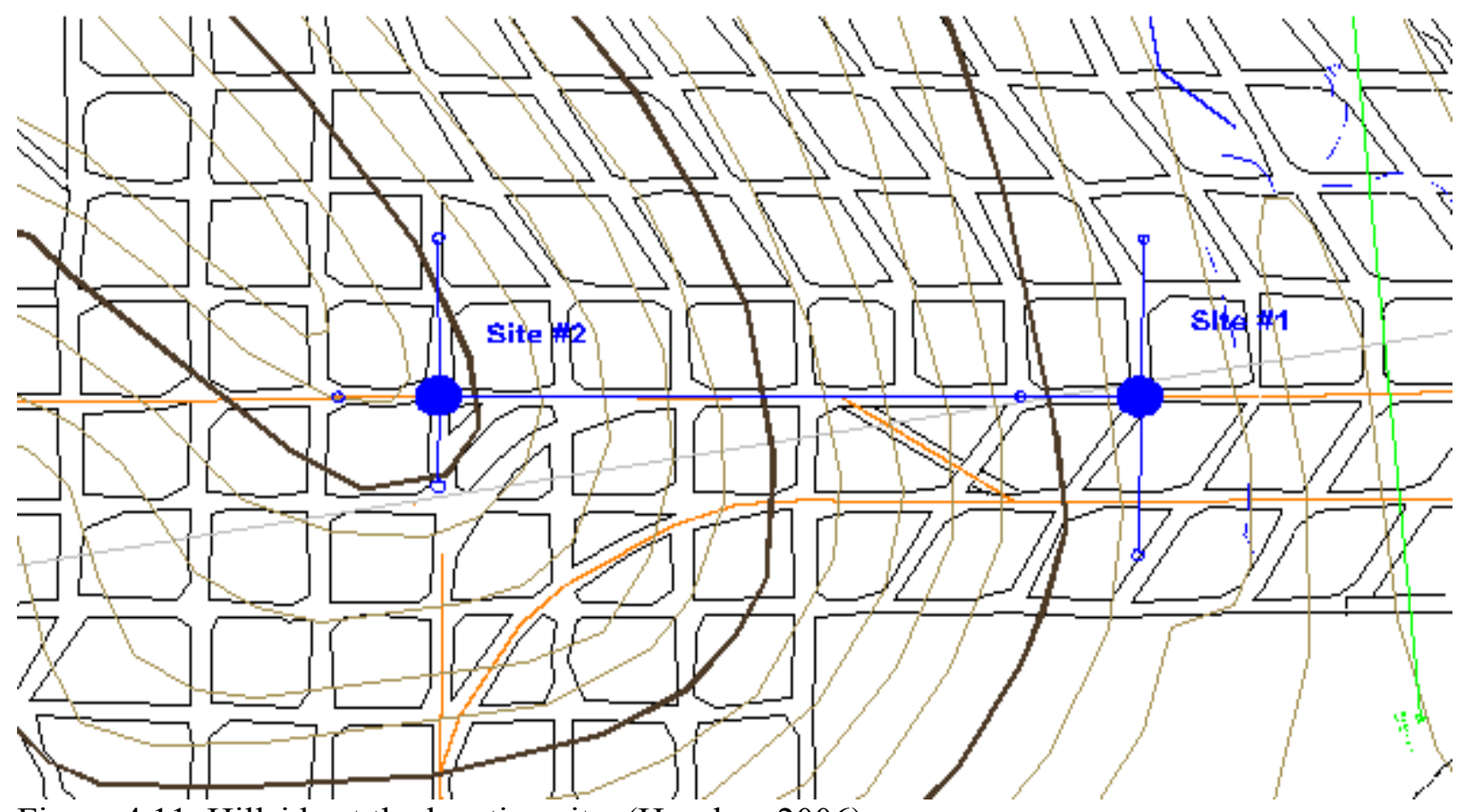

Figure 4.11. Hillside at the location site, (Heasley, 2006). 


\subsubsection{Geophones Locations of the Field Test}

The schematic diagram of the geophones array on the surface at the Federal \#2 Mine is as shown in Figure 4.12. Location \#1 was closest to the spillway of the pond which is by the hillside (see Figure 4.12). Location \#2 was the central location and it was exactly $779 \mathrm{ft}$ above the mine. Location \#4 was up on the hillside. Locations \#1, \#2, \#3, and \#4 were surveyed exactly before the test. Locations \#2a and \#2b were measured using a tape from location \#2, while location \#3a was just estimated. Location \#2a was the second location of the Weir-Jones downhole geophone after the initial set-up. Similarly, the ESG geophone was re-located at location $\# 2 \mathrm{~b}$ after the initial testing. The position of the geophones were obtained using total station surveying instrument prior to the day of the field test, the results are shown below (see Table 4.4). The individual receivers (geophones or digital sensors) were placed in such a manner that they were physically connected to the solid earth. This was accomplished by mounting some where the solid surface was exposed and held in place using one shovel full of the soil. And we employed a handheld auger to bury geophones at a distance below the surface, where geophones were located in mud to maximize coupling to the earth (see Figure 4.13 and Figure 4.14).

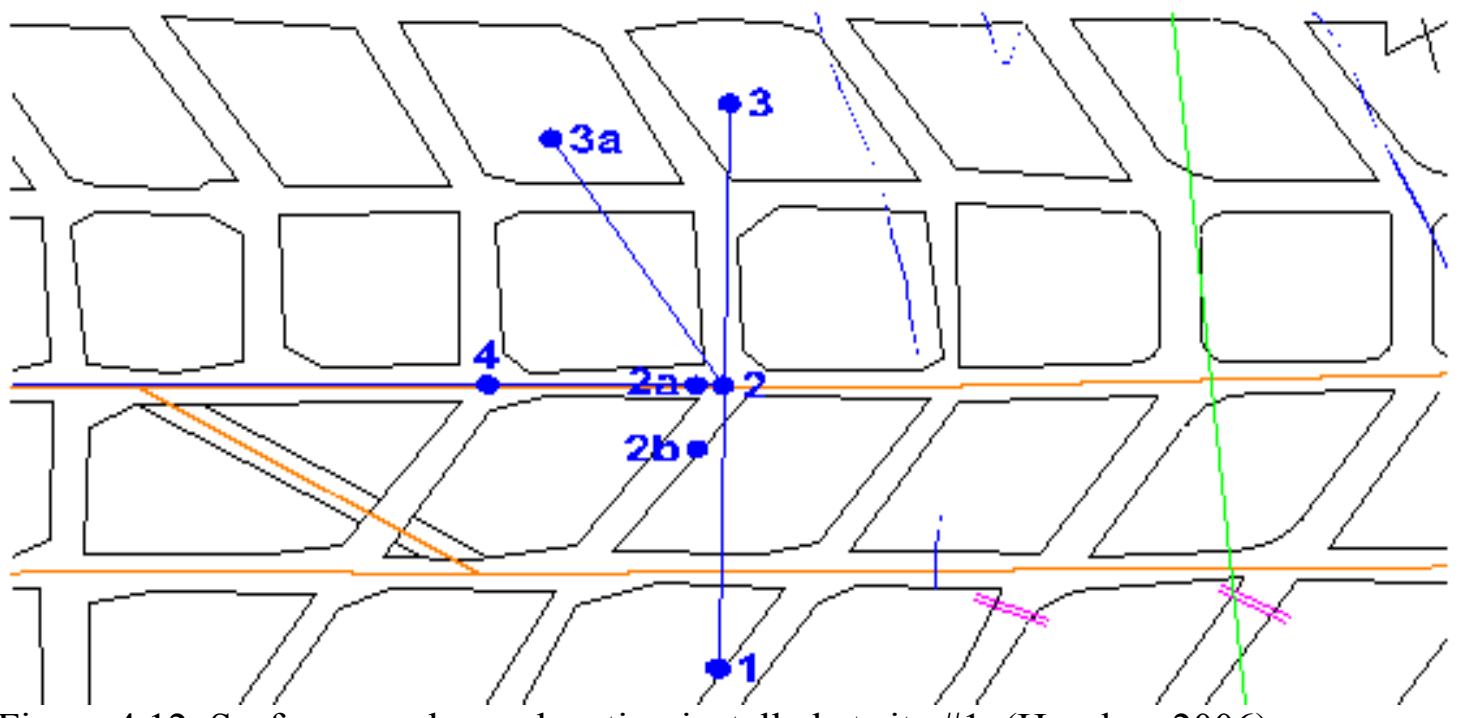

Figure 4.12. Surface geophones location installed at site \#1, (Heasley, 2006). 
Table 4.4. Surface geophone coordinates.

\begin{tabular}{|c|c|c|}
\hline Seismic Point & $\mathbf{X}$ & $\mathbf{Y}$ \\
\hline 1 & $-29,838.45$ & $30,980.35$ \\
\hline 2 & $-29,986.34$ & $31,010.41$ \\
\hline $2 \mathrm{a}$ & $-29,988.44$ & $30,999.10$ \\
\hline $2 \mathrm{~b}$ & $-29,954.92$ & $30,993.38$ \\
\hline 3 & $-30,134.45$ & $31,040.51$ \\
\hline $3 \mathrm{a}$ & $-30,129.66$ & $30,962.42$ \\
\hline 4 & $-30,005.32$ & $30,911.82$ \\
\hline
\end{tabular}

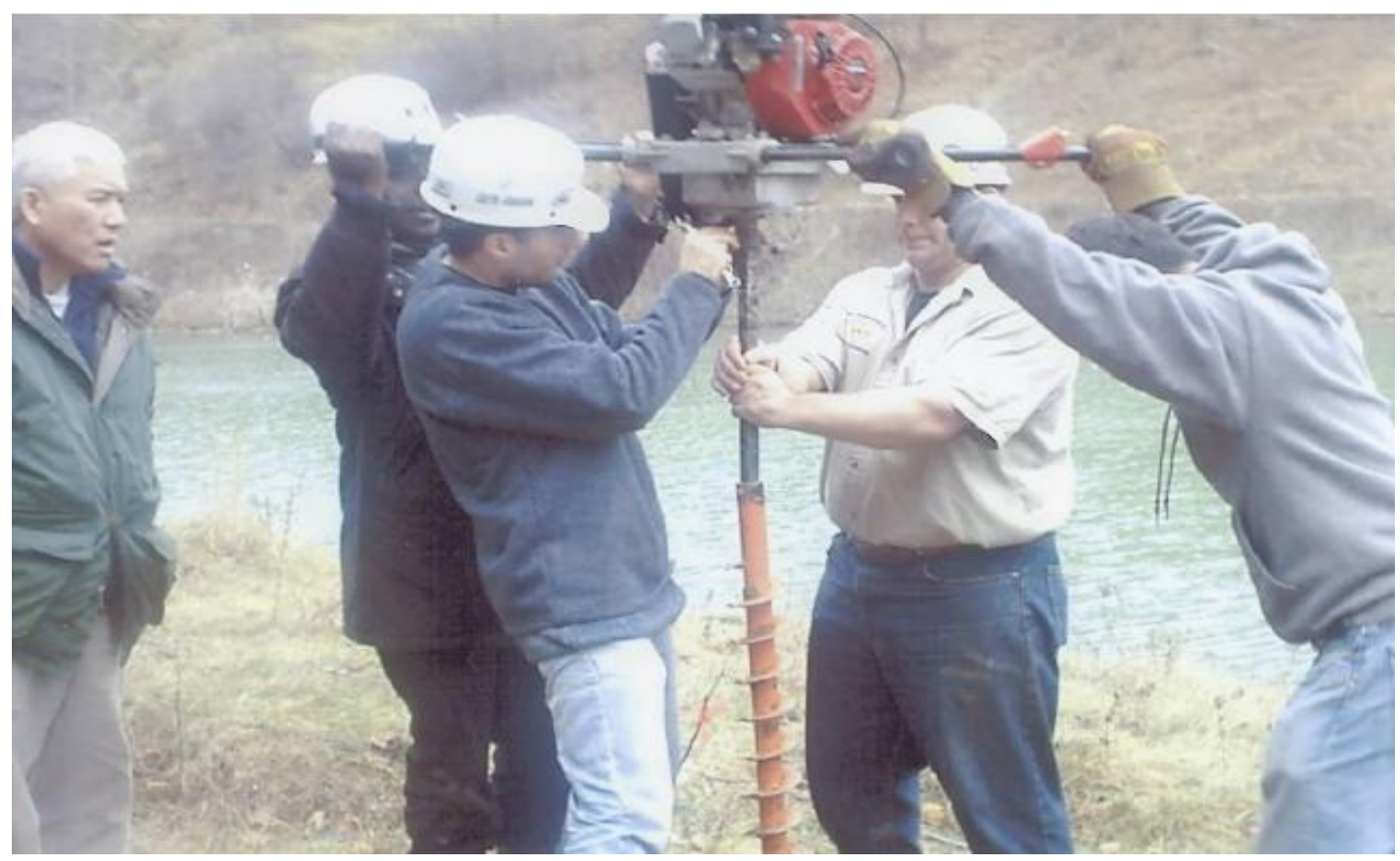

Figure 4.13. Geophone installations using a handheld auger close to the spillway of the pond by the Hillside. 


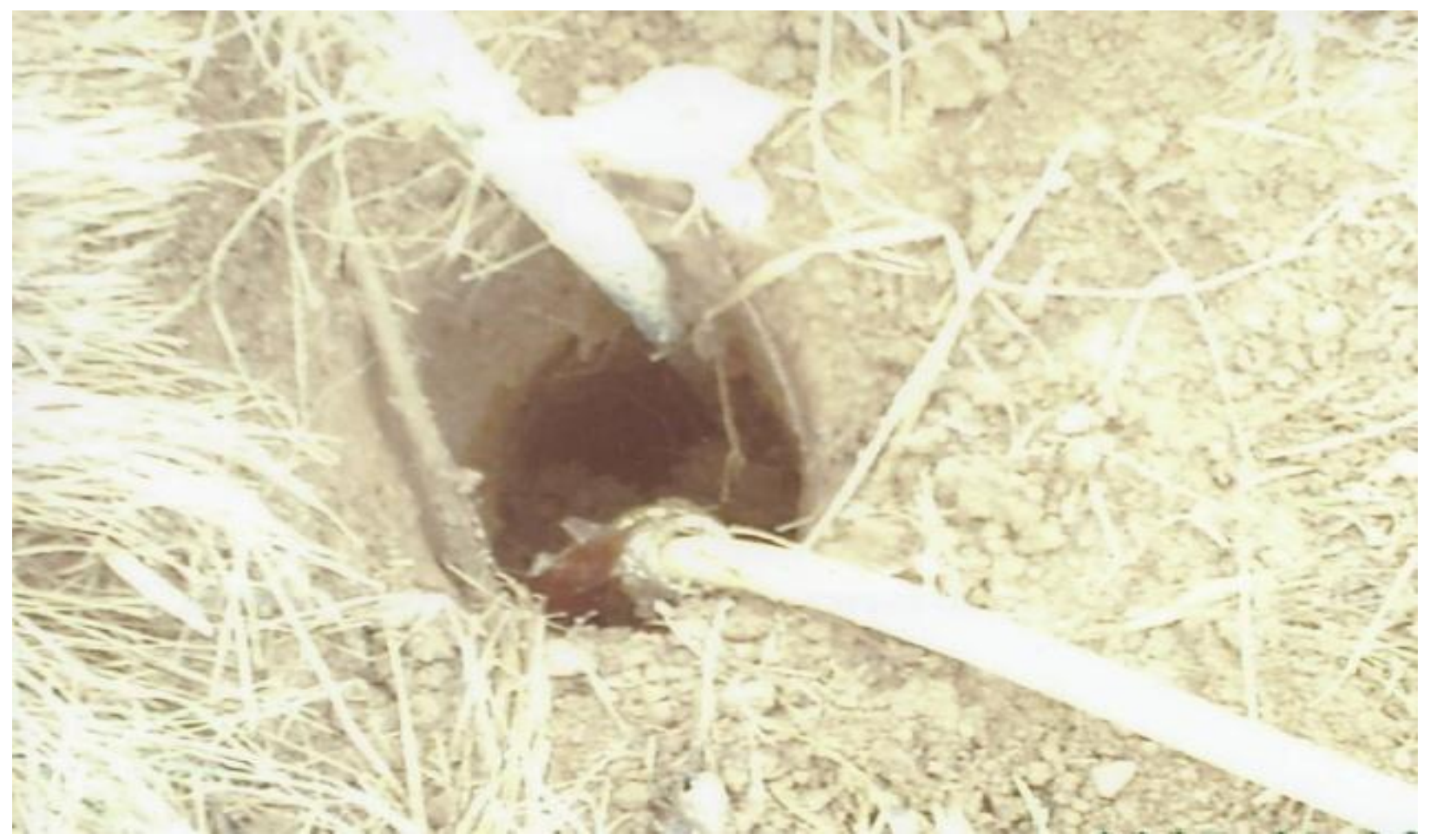

Figure 4.14. Buried geophone installations at the hillside site.

\subsubsection{Test Protocol}

For the underground signaling, two different signaling devices were employed: a sledgehammer and a crib block at two different pounding locations, on a roof bolt (see Figure 4.15) and on the roof rock (Figure 4.16). For each combination of signaling device and location, 5 hits were performed and with a pause of 30 seconds in between each hit. During the signaling, the exact time that the pounding for each device had started was recorded. After a complete pounding cycle, the underground team moved to the next location. 


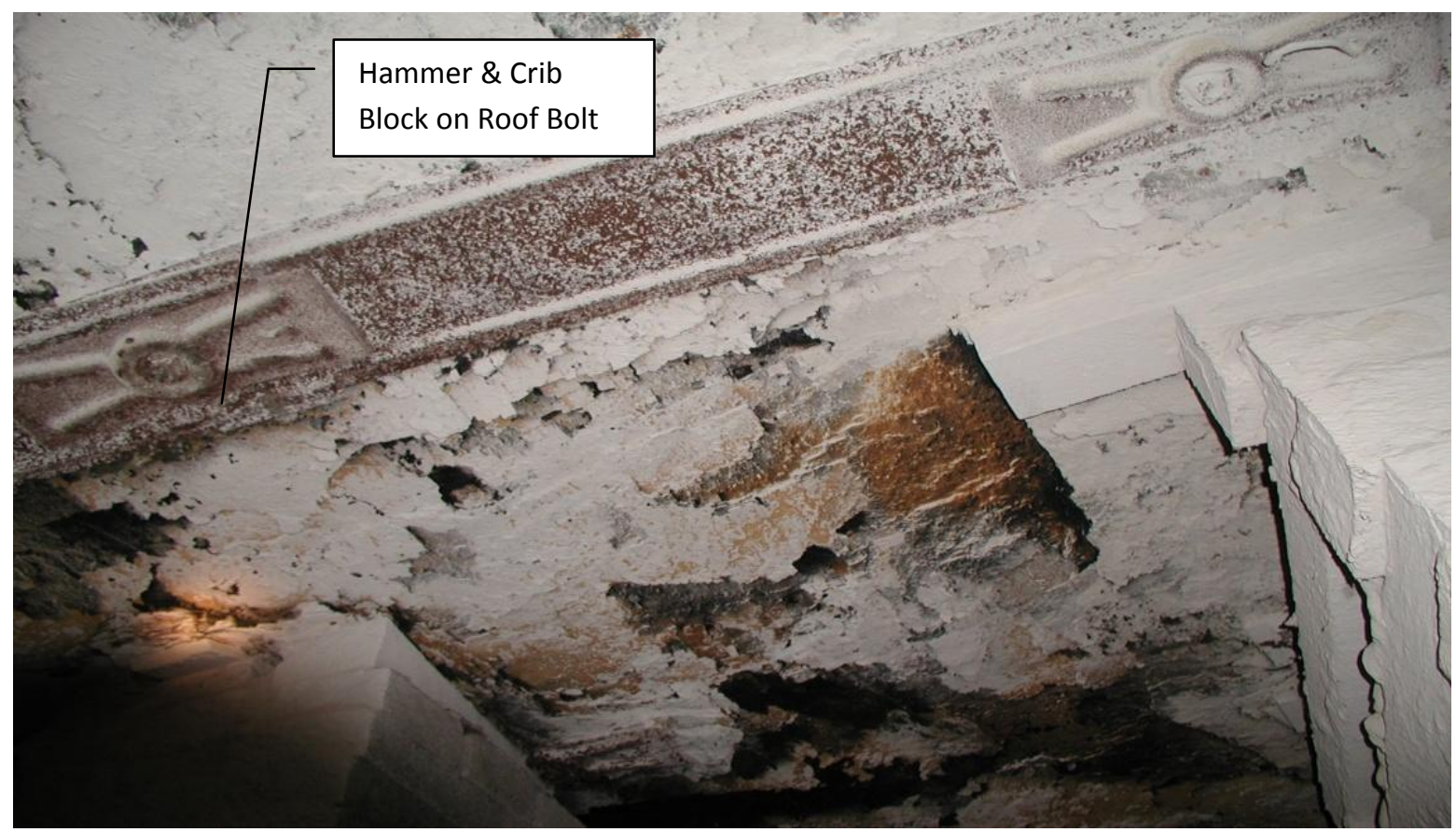

Figure 4.15. Pounding location on the underground roof bolt, (Heasley, 2006).

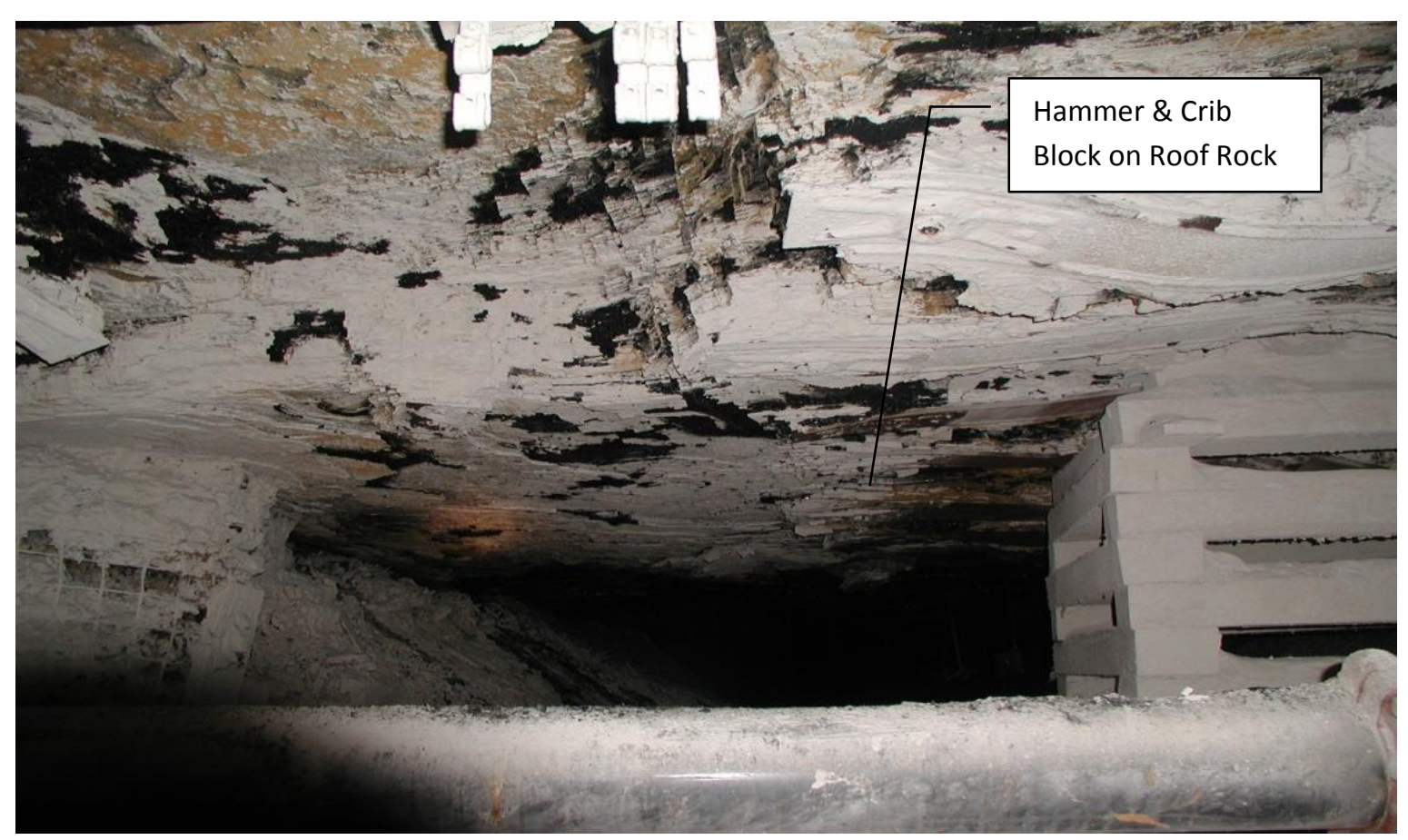

Figure 4.16. Pounding location on the underground mine roof, (Heasley, 2006). 


\subsubsection{Seismic Field Test Results}

Seismic data from the geophone arrays were digitized and collected on computers for analysis. After reviewing the results during the field test, no signals from the underground signaling were observed. To try and improve signal detection some of the geophones were relocated and the underground pounding was done harder and at precise times, but still no signals were detected on the surface. After reviewing the seismic data, we concluded that the signals were not above the background noise at this site.

The background noise environment at the site was highly complex and could have had contributions from many sources. We could hear the bulldozer working on the coal stockpile not too far away. Also, the coal cars used for Federal \#2s bunker system were not too far away underground (2000ft). Any of these sources and other unknown sources could have been interfering with the detection of the pounding signals. Obviously, background noise is a significant consideration in the operation of a practical seismic-based mine location system 


\section{CHAPTER FIVE}

\section{ESTIMATING THE POUNDING FACTOR}

\subsection{Introduction}

In order to ultimately determine the surface particle velocity for a practical situation the pounding factor, $K$, that relate the pounding instruments to the frequency and amplitude of the seismic signal needs to be appropriately determined (Heasley, 2009). This pounding factor is the ratio of the proportionality constant, $g_{0}$, to dwell time $\tau_{s}$. The proportionality constant is the value which relates the surface particle displacement at the point of pounding with the pounding force, while the dwell time is the period that the pounding instrument is in contact with the pounding surface:

$$
\kappa=\frac{g_{0}}{\tau_{s}}
$$

where:

$$
\begin{aligned}
& \mathrm{g}_{\mathrm{o}} \quad=\text { proportionality constant } \\
& \tau_{\mathrm{s}} \quad=\text { dwell time, sec }
\end{aligned}
$$

Because of the uncertainties still associated with the mechanics of the roof momentum, the pounding factor cannot be directly determined from the field experiment alone. However, by employing a study of parallel theoretical analysis with the experimental study, the pounding factor could be determined. To achieve this, a numerical model of the expected seismic attenuation of one of the mines where seismic signal were observed was developed in an EXCEL spreadsheet. The result of the theoretically determined attenuated signal energy of the mine was then back-analyzed with the peak particle velocity measured during the experiment to compute the pounding factor. 


\subsection{Summary of the Experimental Field Test Study}

In the experimental study part of the project, two experimental tests were conducted at two different underground mines. The first test was a relative shallow (440 ft deep) mine while the second was a deeper (779 ft deep) mine and in a noisy environment. Test seismic systems were installed at the mine surface with geophones being installed at various offset distances away from the source. A series of underground seismic signals with pounding devices: crib block and sledgehammer, at different locations were created. However, only the seismic signal at 4-West Mine of Dana Mining were observed between $(0-140)$ feet away from the source (see Table 5.0 for crib block and Table 5.1 for sledgehammer) and utilized to calculate the pounding factor for the crib block and sledgehammer, (Details of these tests are included in the field test report in Chapter 4).

Table 5.0. The peak particle velocity, PPV, recorded for crib block at 4-West Mine of Dana Mining.

\begin{tabular}{|l|l|}
\hline Offset distance, ft & PPV, ft/sec \\
\hline 0 & 0.000297474 \\
\hline 70 & 0.000264559 \\
\hline 100 & 0.0002311053 \\
\hline 122 & 0.000214698 \\
\hline 140 & 0.000163385 \\
\hline
\end{tabular}

Table 5.1. The peak particle velocity, PPV, recorded for sledgehammer at 4-West Mine of Dana Mining.

\begin{tabular}{|c|c|}
\hline Offset distance, ft & PPV, ft/sec \\
\hline 0 & 0.000164875 \\
\hline 70 & 0.000146102 \\
\hline 100 & 0.000124256 \\
\hline 122 & 0.000100623 \\
\hline
\end{tabular}




\subsection{Theoretical Analysis of the Field Study and Pounding Factor Computation}

5.2.1 Derivation of Pounding Factor Equation: First, a mathematical derivation of the pounding factor needs to be established. This is achieved by employing equation (5.1). As previously shown in equation (3.35), equation (5.1) is the radial particle velocity-time function, $\mathrm{V}_{\mathrm{r}}(\mathrm{t})$, for a remote point in a layered medium due to the miner's pounding on the roof (Details of the equation are included in chapter 3), (Heasley, 2009).

$$
\mathrm{v}_{\mathrm{r}}(\mathrm{t})=\frac{\cos \theta}{4 \pi \rho_{1} \mathrm{v}_{1}^{2}} \times \frac{1}{\mathrm{R}} \times \mathrm{e}^{-\sum_{1}^{\mathrm{n}} \alpha_{\mathrm{n}} \mathrm{r}_{\mathrm{n}}} \times \mathrm{T}_{\mathrm{n}} \times \frac{\mathrm{g}_{0} 2 \pi}{\tau_{\mathrm{S}}} \cos \left(\frac{2 \pi}{\tau_{\mathrm{S}}}\left(\mathrm{t}-\sum_{1}^{\mathrm{n}} \frac{\mathrm{r}_{\mathrm{n}}}{\mathrm{v}_{\mathrm{n}}}\right)\right)
$$

For the peak particle velocity on the surface:

$$
\cos \left(\frac{2 \pi}{\tau_{\mathrm{S}}}\left(\mathrm{t}-\sum_{1}^{\mathrm{n}} \frac{\mathrm{r}_{\mathrm{n}}}{\mathrm{v}_{\mathrm{n}}}\right)\right)=1
$$

(since at a peak particle velocity, $(\mathrm{t}-\mathrm{R} / \mathrm{V})=0$ and $\operatorname{Cos} 0=1)$, therefore, making the surface peak particle velocity for a remote point due to the miner's pounding on the roof:

$$
\mathrm{v}_{\mathrm{r}}=\frac{\cos \theta}{4 \pi \rho_{1} \mathrm{v}_{1}^{2}} \times \frac{1}{\mathrm{R}} \times \mathrm{e}^{-\sum_{1}^{\mathrm{n}} \alpha_{\mathrm{n}} \mathrm{r}_{\mathrm{n}}} \times \mathrm{T}_{\mathrm{n}} \times \frac{\mathrm{g}_{0} 2 \pi}{\tau_{\mathrm{S}}}
$$

simplifying and solving for $\left[\mathrm{g}_{0} / \tau_{\mathrm{s}}\right]$, we see that the pounding factor, $\kappa$, is;

$$
\kappa=\left[\frac{g}{\tau_{s}}\right]=\mathrm{v}_{\mathrm{r}}(\mathrm{t}) \times\left(\frac{\cos \theta}{2 \rho_{1} \mathrm{v}^{2}} \times \frac{1}{\mathrm{R}} \times \mathrm{T}_{\mathrm{n}} \times \mathrm{e}^{\sum_{1}^{\mathrm{n}} \alpha_{\mathrm{n}} \mathrm{r}_{\mathrm{n}}}\right)^{-1}
$$

where;

$$
\begin{aligned}
& \kappa=\text { pounding factor value for a given pounding instrument and location } \\
& \mathrm{V}_{\mathrm{r}}(\mathrm{t})=\text { peak particle velocity recorded at the surface geophones }
\end{aligned}
$$


and:

$$
\left(\frac{\cos \theta}{2 \rho_{1} \mathrm{~V}^{2}} \times \frac{1}{\mathrm{R}} \times \mathrm{T}_{\mathrm{n}} \times \mathrm{e}^{\sum_{1}^{\mathrm{n}} \alpha_{\mathrm{n}} \mathrm{r}_{\mathrm{n}}}\right)
$$

represent the signal loss into the medium. From equation (5.3) above, we can conclude that the pounding factor can be determined by computing the ratio of the peak particle velocity measured from the geophones and the value which represent the signal loss

Since we already have the seismic signal at the 4-West Mine of Dana Mining which were observed between $(0-140)$ feet away from the source (see Table 5.1), it became reasonable that the signal loss in the overburden of the mine can be determined. This was achieved by modeling the seismic signal loss at the 4-West Mine of Dana Mining in an EXCEL spreadsheet.

\subsubsection{Attenuation Modeling of 4-West Mine of Dana Mining in an EXCEL Spreadsheet}

The seismic attenuation of the 4-West Mine of Dana Mining was modeled using the EXCEL spreadsheet. The spreadsheet consists of two parts; input parameters and output parameters. For the input parameters, there are five inputs which include; mine depth, sonic velocity and density of the pounding location, offset distances of the geophone from the pounding source and geological parameters of each layer in the overburden as shown in Figure 5.1. The seismic geologic parameters input of each layer which include: sonic velocity, layer's thickness, density and attenuation coefficient was determined from the drill log of the nearby ventilation shaft (see Table 4.1) and the seismic properties of rocks in Table 3.1. Table 3.1, contains average values of the seismic parameters needed to model the mine overburden in order to determine the expected signal loss (Details of these properties are included in Chapter 2). 
The output parameters of the modeling in the EXCEL spreadsheet are shown in Figure 5.2. The computations which are involved in each of the output column for each layer/strata of the mine overburden are described below.

\begin{tabular}{|c|c|c|c|c|}
\hline \multicolumn{5}{|c|}{ INPUT PARAMETER } \\
\hline \multicolumn{2}{|c|}{ Mine Geological Input } & & Geophones & Offset Distance, $\mathrm{ft}$ \\
\hline Mine Depth,ft & 441.00 & & Geophone \#1 & 0.00 \\
\hline Roof Density,lbs/ft^3 & 143.58 & & Geophone \#2 & 70.00 \\
\hline Roof P wave Velocity, ft/sec & 8393.00 & & Geophone \#3 & 100.00 \\
\hline & & & Geophone \#4 & 122.00 \\
\hline & & & Geophone \#5 & 140.00 \\
\hline Number of la & ers & 28 & \multicolumn{2}{|c|}{ Select layers } \\
\hline \multicolumn{5}{|c|}{ Mine Layers' Geological Input } \\
\hline LAYERS' NAME & Thickness, ft & $\begin{array}{c}\text { P-wave Velocity, } \\
\mathrm{ft} / \operatorname{secs}(\mathrm{V})\end{array}$ & Density, Ibs/ft^3 & $\begin{array}{c}\text { Attenuation Coff, } \\
\text { nepers/ft }\end{array}$ \\
\hline SUBSOIL & 10 & 3000 & 56.19 & 0.006545 \\
\hline SANDSTONE & 36.5 & 8393 & 143.58 & 0.000785 \\
\hline SHALE & 37 & 3937 & 146.71 & 0.000628 \\
\hline COAL & 8 & 5249 & 68.02 & 0.000055 \\
\hline SHALE & 25 & 3937 & 146.71 & 0.000628 \\
\hline LIMESTONE & 25 & 6234 & 165.43 & 0.000187 \\
\hline
\end{tabular}

Figure 5.1. Input portion of the EXCEL spreadsheet. 


\begin{tabular}{|c|c|c|c|c|c|}
\hline Medium Impedance & Radial Distance & Signal time travel & \multicolumn{3}{|c|}{ Signal Energy Losses } \\
\hline $\mathrm{lbs} / \mathrm{ft} \wedge 2 . \mathrm{secs}$ & $\mathrm{ft}$ & sec & $\begin{array}{c}\text { Geometric } \\
\text { Spreading, }(1 / \mathrm{ft})\end{array}$ & $\begin{array}{l}\text { Hysteresis, } \\
\text { (nepers/ft) }\end{array}$ & \begin{tabular}{|c} 
Inter-Bed Reflection \\
/Transmission
\end{tabular} \\
\hline \multirow[t]{2}{*}{168555.493282} & 10 & 0.003333333 & 0.1 & 0.06545 & \\
\hline & & & & & 0.245411041 \\
\hline \multirow[t]{2}{*}{1205103.106207} & 36.5 & 0.004348862 & 0.02739726 & 0.0286525 & \\
\hline & & & & & 1.352009307 \\
\hline \multirow[t]{2}{*}{577581.524955} & 37 & 0.009398 & 0.027027027 & 0.023236 & \\
\hline & & & & & 1.235974755 \\
\hline \multirow[t]{2}{*}{357035.501525} & 8 & 0.001524 & 0.125 & 0.000436 & \\
\hline & & & & & 0.764025245 \\
\hline \multirow[t]{2}{*}{577581.524955} & 25 & 0.00635 & 0.04 & 0.0157 & \\
\hline & & & & & 0.718014004 \\
\hline 1031249.283030 & 25 & 0.004010526 & 0.04 & 0.004675 & \\
\hline
\end{tabular}

Figure 5.2. Output portion of the EXCEL spreadsheet.

\subsubsection{Impedance}

Impedance is the product of sonic velocity and the density. This parameter greatly influences the magnitude of the signal that would be reflected or refracted at the interfaces. A mathematical formulation for impedance is:

$$
\text { Impedance }=\mathrm{V} \times \rho
$$

where :

$$
\begin{aligned}
& \mathrm{V} \quad=\text { sonic velocity, } \mathrm{ft} . / \mathrm{sec} \\
& \rho \quad=\text { density, lbs. } / \mathrm{ft}^{3}
\end{aligned}
$$

\subsubsection{Angle of Refraction and Transmission}

When a miner pounds underground, the signal generated travels to the surface through different layers. At each of these layers, part of the signal would be transmitted while the remaining part is refracted. By applying Snell's law, these angles of refraction and transmission 
can be determined as shown below. The knowledge of these angles is going to enable us to calculate the following: 1) actual angular distance traveled to the surface, 2) the signal travel time and 3) the signal energy partition at each interface (transmission coefficient).

$$
\begin{aligned}
& \sin \theta_{\mathrm{t}}=\frac{\mathrm{v}_{1}}{\mathrm{v}_{2}} \sin \theta_{\mathrm{i}} \\
& \sin \theta_{\mathrm{i}}=\frac{\mathrm{v}_{2}}{\mathrm{v}_{1}} \sin \theta_{\mathrm{t}}
\end{aligned}
$$

where:

$$
\begin{array}{ll}
\theta_{\mathrm{t}} & =\text { transmission angle, degree } \\
\theta_{\mathrm{i}} & =\text { incidence angle, degree } \\
\mathrm{v}_{1} & =\text { velocity of the incident medium, } \mathrm{ft} / \mathrm{sec} \\
\mathrm{v}_{2} & =\text { velocity of the refracted medium, } \mathrm{ft} / \mathrm{sec}
\end{array}
$$

\subsubsection{Offset and Radial Distance}

Offset distance is the horizontal distance between the underground signaling location and the surface geophone. Radial distance is the distance between the source and the geophone. In the Figure 5.3 below, $(\mathrm{D} 1+\mathrm{D} 2)$ is the offset distance and $(\mathrm{L} 1+\mathrm{L} 2)$ is the radial distance.

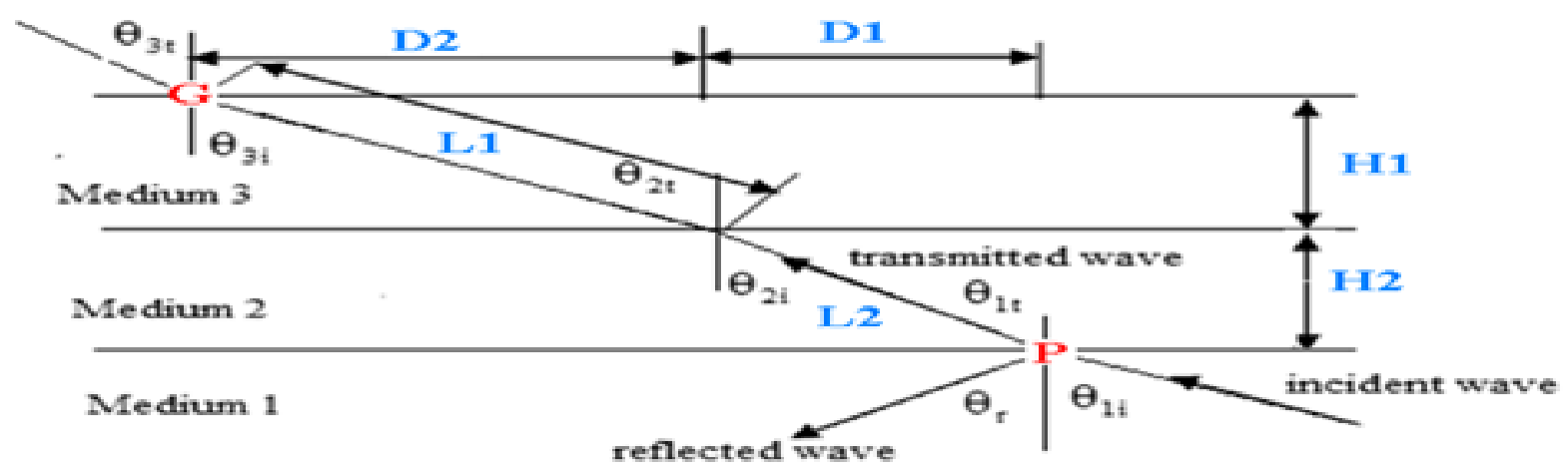

Figure 5.3. Signal reflection and transmission at the interfaces of layered medium. 
where:

$$
\begin{array}{ll}
\mathrm{G} & =\text { surface geophone location } \\
\mathrm{P} & =\text { underground pounding location } \\
\mathrm{H} 1 & =\text { thickness of layer } \\
\mathrm{H} 2 & =\text { thickness of the preceding layer } \\
\theta_{\mathrm{ni}} & =\text { incidence angle }(\mathrm{n}=\text { layers of rock-1, 2, 3, etc }) \\
\theta_{\mathrm{nt}} & =\text { refracted angle }(\mathrm{n}=\text { layers of rock-1, } 2,3, \text { etc })
\end{array}
$$

Employing the angle of incidence at each layer, the radial distance, L1 and L2, of each layer can be determined by multiplying the cosine of the incidence angle with the height of the strata (see equation (5.8)). Also, the offset distance, $\mathbf{D} 1$ and $\mathrm{D} 2$, is the product of the tangent of the incidence angle with the strata height, $\mathrm{H} 1$ and $\mathrm{H} 2$, (see equation (5.9)).

The radial distance travelled by the signal between each layer is;

$$
L_{n}=\frac{H_{n}}{\cos \theta_{n i}}
$$

Offset distance at each layer of the medium is;

$$
D_{n}=H_{n} \tan \theta_{n i}
$$

where;

$$
\begin{aligned}
& D_{n} \quad=\text { offset distance at each rock layer } \\
& H_{n} \quad=\text { height of the rock layer }
\end{aligned}
$$




\subsubsection{Signal Travel Time}

This is calculated by dividing the velocity of signal travel with the distance that signal covers within each layer of the mine overburden.

$$
\mathrm{t}_{\mathrm{i}}=\frac{\mathrm{v}_{\mathrm{i}}}{\mathrm{r}_{\mathrm{i}}}
$$

where:

$$
\begin{aligned}
& \mathrm{v}_{\mathrm{i}} \quad=\text { velocity of travel, } \mathrm{ft} / \mathrm{s} \\
& \mathrm{r}_{\mathrm{i}} \quad=\text { radial distance travel within ith layer, } \mathrm{ft}
\end{aligned}
$$

\subsubsection{Angle of Incidence at the Pounding Surface (initial incident angle)}

Upon the establishment of the offset distance of the geophone, the initial angle of incidence at the pounding surface can be computed using equation (5.10) below:

$$
\theta=\left(\frac{\sum_{n=1}^{N} D_{n}}{\sum_{n=1}^{N} H_{n}}\right) \tan ^{-1}
$$

where:

$$
\begin{array}{ll}
\mathrm{D}_{\mathrm{n}} & =\text { offset distance at each rock layer } \\
\mathrm{H}_{\mathrm{n}} & =\text { height of the rock layer } \\
\mathrm{n} & =\text { number of layers } \\
\mathrm{N} & =\text { total number of layers } \\
\theta & =\text { incident angles at the pounding surface }
\end{array}
$$

In the spreadsheet, the angle of incidence is determined by developing a simple EXCEL macro using equation (5.10) and the EXCEL toolbox: scrollbar. The scrollbar as shown in Figure 5.4 is 
programmed with the equation (5.10), such that, as the offset distance changes (varied) the program automatically computes the new angle of incidence that correspond to the offset distance and the mine height. The purpose is to allow the user to calibrate the angle of incidence at the pounding surface with the already established actual offset distance of the geophone by scrolling. The user would have to scroll until the offset distance matches the geophone offset distance and then record the corresponding angle of incidence.

\begin{tabular}{|c|c|c|c|c|c|c|}
\hline \multicolumn{7}{|c|}{ Calibrating the angle of incidence } \\
\hline Calibrated incidence angle & 0 & 14.07 & 19.43 & 23.55 & 26.85 \\
\hline & & 1 & & & & \\
\hline & & & & & 1 & \\
\hline & OUTPUT-OFFSE \& RADIAL DISTANCE \\
\hline Offset Dist., ft & 0 & 70 & 100 & 122 & 140 \\
\hline Actual Offset dist., ft & 0 & 70.00344246 & 100.0074485 & 122.0048174 & 140.0088093 \\
\hline Radial Dist., ft & 441 & 446.5215358 & 452.1974013 & 457.5654876 & 462.6915459 \\
\hline
\end{tabular}

Figure 5.4. Calibrating the angle of incidence at the pounding surface (initial incident angle).

\subsubsection{Results of the Attenuation model of the 4-West Mine of Dana Mining}

Having calculated the Impedance, Angle of Refraction, Incidence Angle, and Radial distance, their values are then utilized to calculate the expected signal attenuation of the signal. The signal attenuation, which was calculated at the various offset distances; $0 \mathrm{ft}, 70 \mathrm{ft}, 100 \mathrm{ft}$, $122 \mathrm{ft}, 140 \mathrm{ft}$, is shown in the Table 5.4. For each offset distance, three mechanism through which signal is being lost were considered which include (1) energy dissipation/hysteresis, (2) energy partitioned at each layers boundary and (3) geometric spreading. (Details of seismic attenuation are included in Chapter 3). 3-D geophones were used for the experiment, since only the vertical component is recorded, it became reasonable that the vertical component of the energy partition, 
dissipation and geometric spreading is computed using equation 5.3. This is achieved by multiplying the results of the calculation with the cosine of the angle of incidence (Table 5.3).

Table 5.2 Results of the attenuation model, mine depth: $441 \mathrm{ft}$.

\begin{tabular}{|c|c|c|c|c|c|}
\hline Offset distance, $\mathrm{ft}$ & 0 & 70 & 100 & 122 & 140 \\
\hline Radial distance, $\mathrm{ft}$ & 441 & 446.5215 & 452.1974 & 457.5654 & 462.6915 \\
\hline Angle of incidence & 0 & 14.07 & 19.43 & 23.55 & 26.85 \\
\hline Energy partition & 0.0848136 & 0.0793484 & 0.0745189 & 0.0720700 & 0.0656229 \\
\hline Energy dissipation & 0.7165470 & 0.7133851 & 0.7104730 & 0.7089486 & 0.7047582 \\
\hline Geometric spreading & 0.0022675 & 0.0022395 & 0.0022114 & 0.0021977 & 0.0021612 \\
\hline
\end{tabular}

Table 5.3 Computing the vertical component of the energy partition, dissipation and geometric spreading using equation 5.3.

\begin{tabular}{|c|c|c|c|c|c|}
\hline Angle of incidence & 0 & 14.07 & 19.43 & 23.55 & 26.85 \\
\hline Cosine of the incident angle & 1 & 0.9699 & 0.9430 & 0.9167 & 0.8921 \\
\hline Vertical component & $6.812 \mathrm{E}-15$ & $6.078 \mathrm{E}-15$ & $5.458 \mathrm{E}-15$ & $4.896 \mathrm{E}-15$ & $4.408 \mathrm{E}-15$ \\
\hline
\end{tabular}

\subsection{Pounding Factor for crib block, $\kappa_{\text {(crib-block) }}$}

To determine the pounding factor for crib block, the peak particle velocity recorded at the 4West Mine of Dana Mining (see Table 5.0) together with the theoretically determined vertical component of the energy partition, dissipation and geometric spreading (see Table 5.2) were employed. This was achieved by back-analyzing the peak particle velocity measured at each offset distance with the calculated attenuated signal of the mine in equation (5.3), (Table 5.4). 
Table 5.4. Calculating the pounding factor of crib block using PPV recorded from crib block pounding.

\begin{tabular}{|c|c|c|c|}
\hline Offset distance, ft & Vertical component & PPV, ft/sec & Pounding factor \\
\hline 0 & $6.81241 \mathrm{E}-15$ & 0.000297474 & $4.37 \mathrm{E}+10$ \\
\hline 70 & $6.07873 \mathrm{E}-15$ & 0.000264559 & $4.35 \mathrm{E}-10$ \\
\hline 100 & $5.45826 \mathrm{E}-15$ & 0.0002311053 & $4.23 \mathrm{E}+10$ \\
\hline 122 & $4.89675 \mathrm{E}-15$ & 0.000214698 & $4.38 \mathrm{E}+10$ \\
\hline 140 & $4.40847 \mathrm{E}-05$ & 0.000163385 & $3.71 \mathrm{E}+10$ \\
\hline
\end{tabular}

From the Table 5.4, the average value of the pounding factor is: 4.21E10

\subsection{Pounding Factor for sledgehammer, $\kappa_{(\text {hammer })}$}

Similarly, the pounding factor for a sledgehammer is determined by back analyzing the peak particle velocity resulting from the sledgehammer (see Table 5.1) and the theoretically determined vertical component of the energy partition, dissipation and geometric spreading (see Table 5.2) in Equation (5.3) (see Table 5.5)

Table 5.5. Calculating the pounding factor of sledgehammer using PPV recorded from sledgehammer pounding.

\begin{tabular}{|c|c|c|c|}
\hline Offset distance, $\mathrm{ft}$ & vertical component & $\mathrm{PPV}, \mathrm{ft} / \mathrm{sec}$ & Pounding factor \\
\hline 0 & $6.81241 \mathrm{E}-15$ & 0.000164875 & $2.42 \mathrm{E}+10$ \\
\hline 70 & $6.07893 \mathrm{E}-15$ & 0.000146102 & $2.40 \mathrm{E}+10$ \\
\hline 100 & $5.45826 \mathrm{E}-15$ & 0.000124256 & $2.28 \mathrm{E}+10$ \\
\hline 122 & $4.89675 \mathrm{E}-15$ & 0.000100623 & $2.05 \mathrm{E}+10$ \\
\hline
\end{tabular}

From the Table 5.5, the average pounding factor for sledgehammer is $2.29 E 10$ 


\subsection{Validating the Results of the Pounding Factors}

In order to validate the results of the pounding facto, $\kappa$ for both the crib block and the sledgehammer, the comparison plot between the theorectically determined peak particle velocity and the experimental peak particle velocity were plotted as shown in Table 5.6 and Table 5.7 which are plotted in Figure 5.5 and Figure 5.6.

Table 5.6. Experimental and theoretical values of the PPV for sledgehammer.

\begin{tabular}{|c|c|c|}
\hline$\underline{\text { Offset,ft }}$ & Experimental PPV, ft/sec & Theoretical PPV,ft/sec \\
\hline 0 & 0.000164875 & $1.56 \mathrm{E}-04$ \\
\hline 70 & 0.000146102 & $1.39 \mathrm{E}-04$ \\
\hline 100 & 0.000124256 & $1.25 \mathrm{E}-04$ \\
\hline 122 & 0.000100623 & $1.12 \mathrm{E}-04$ \\
\hline
\end{tabular}

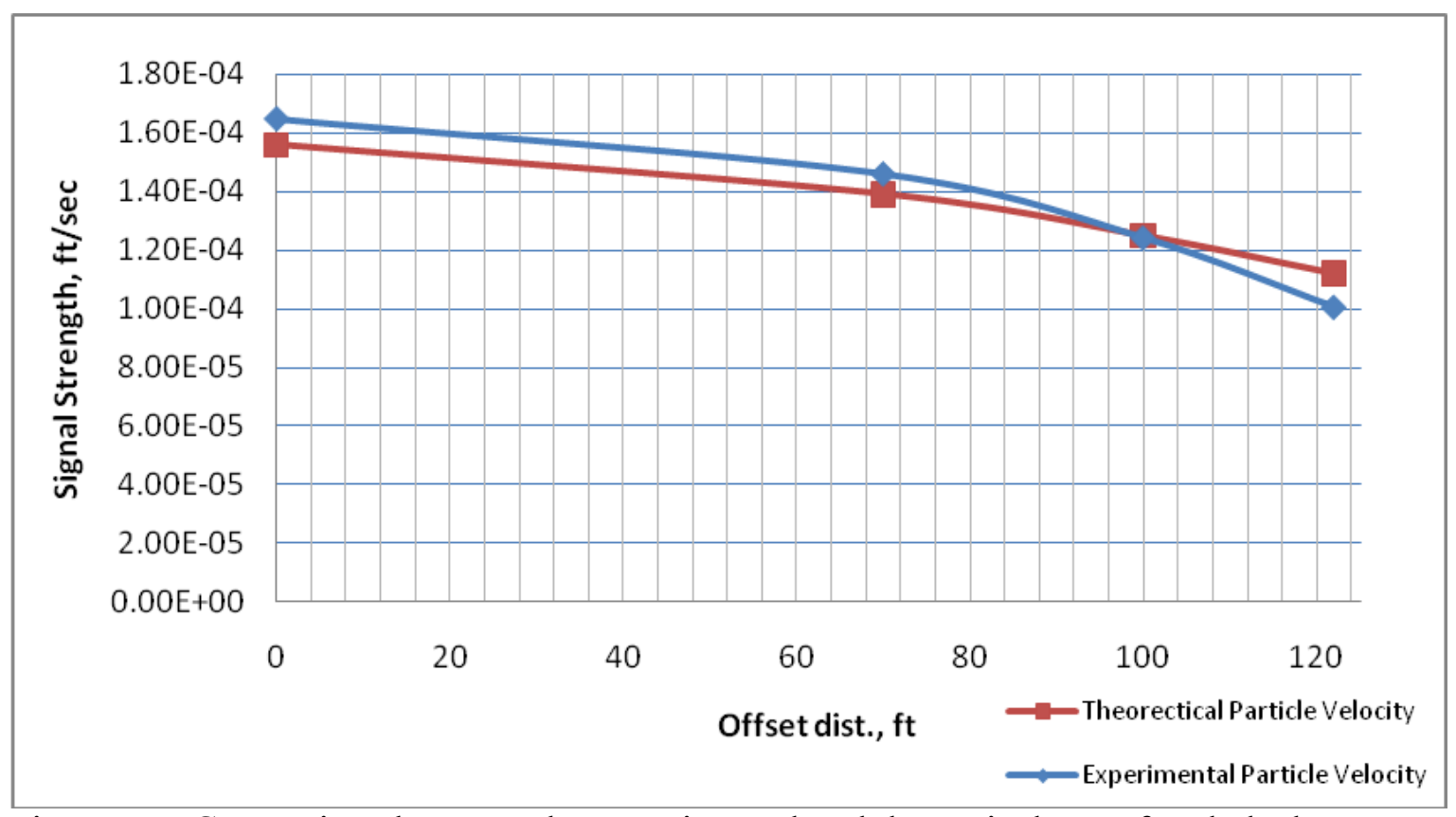

Figure 5.5. Comparison between the experimental and theoretical PPV for sledgehammer 
Table 5.7 Experimental and theoretical values of the PPV for crib block.

\begin{tabular}{|c|c|c|}
\hline Offset distance, $\mathrm{ft}$ & $\begin{array}{l}\text { Experimental peak } \\
\text { particle velocity, ft/sec }\end{array}$ & $\begin{array}{l}\text { Theoretical peak } \\
\text { particle velocity,ft/sec }\end{array}$ \\
\hline 0 & 0.000297474 & $2.95 \mathrm{E}+04$ \\
\hline 70 & 0.000264559 & $2.63 \mathrm{E}-04$ \\
\hline 100 & 0.0002311053 & $2.37 \mathrm{E}+04$ \\
\hline 122 & 0.000214698 & $2.12 \mathrm{E}+04$ \\
\hline 140 & 0.000163385 & $1.91 \mathrm{E}+04$ \\
\hline
\end{tabular}

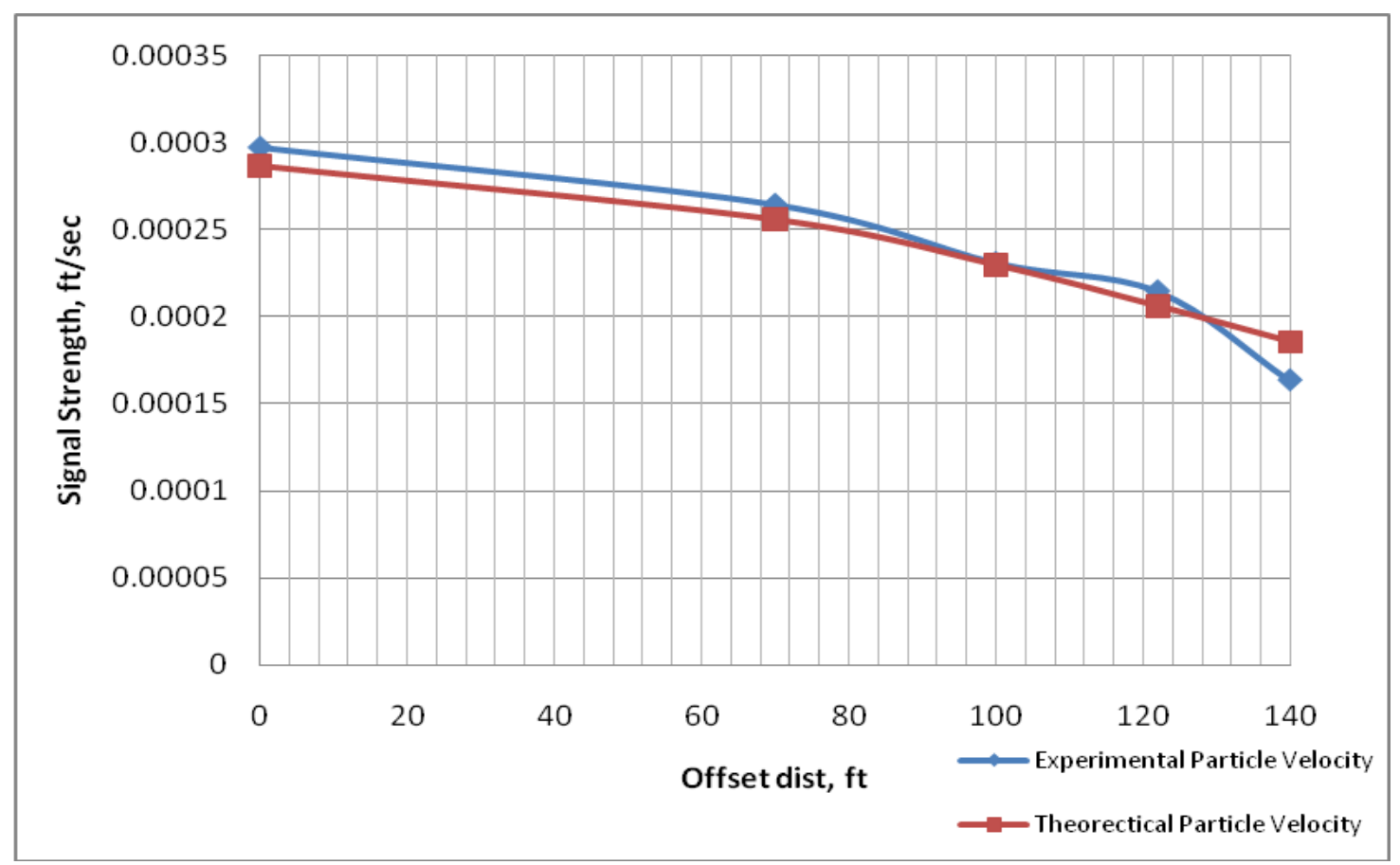

Figure 5.6. Comparison between the experimental and theoretical PPV for crib block.

\subsection{Conclusion on the Validation Results}

The fit obtained between the calculated and observed peak particle velocity (Figure 5.5 and Figure 5.6) provides assurance that the pounding factor (for both crib block and sledgehammer) relating the pounding instruments to the frequency and amplitude of the seismic signal is fairly 
reliable. These values, 4.21E10 for crib block and 2.29E10 for sledgehammer can form a basis for calculating the expected peak particle velocity on the surface of a given mine in the future.

\section{7 Calculating the Surface Peak Particle Velocity Using the EXCEL Program:}

This program was designed to be simple. The program uses a mine specific geology with the appropriate average seismic parameters, and geophone horizontal offset distance and the calculated pounding factor to numerically model the expected seismic attenuation and associated peak particle velocities. With the expected seismic attenuation of a given mine and the pounding factor (which has been described in this chapter), the EXCEL program can predict the expected surface peak particle velocity (seismic signal) from a miner who pounds underground.

This EXCEL program requires that the user has access to the geologic information of each layer/strata in the mine overburden. This geological information which includes the layers' name and height can be reasonably determined from the drilling log of the closest drill hole to the area of interest. With the type of each stratum known, the user can then look for the seismic parameters of each stratum in the Table 3.1.

The roof seismic parameters, that is, the density and compressional velocity of the immediate roof where the miners are being trapped can then be input. After the roof seismic properties have been input, the user is then required to select between the two available options of pounding devices: a sledgehammer or a crib block. To input the seismic and geological parameters of each layer, the user can either click the "prompt for data" button and type in the data or go directly into the spreadsheet column. The Offset Distance where the user desires to know the peak particle velocity is then selected by scrolling the bar as shown below (see Figure 5.7). By clicking the "CALCULATE BUTTON" the program will automatically calculates the surface peak particle velocity at that location (see Figure 5.8). Apart from the surface peak particle velocity, the 
program can also generate a close representation of the expected signal waveform by clicking on the "update-waveform" button (see Figure 5.8). After clicking the update-waveform button, the user can make adjustments to the signal waveform by varying the phase of the signal using the scrollbar until a waveform that closely looks like the expected signal is shown.

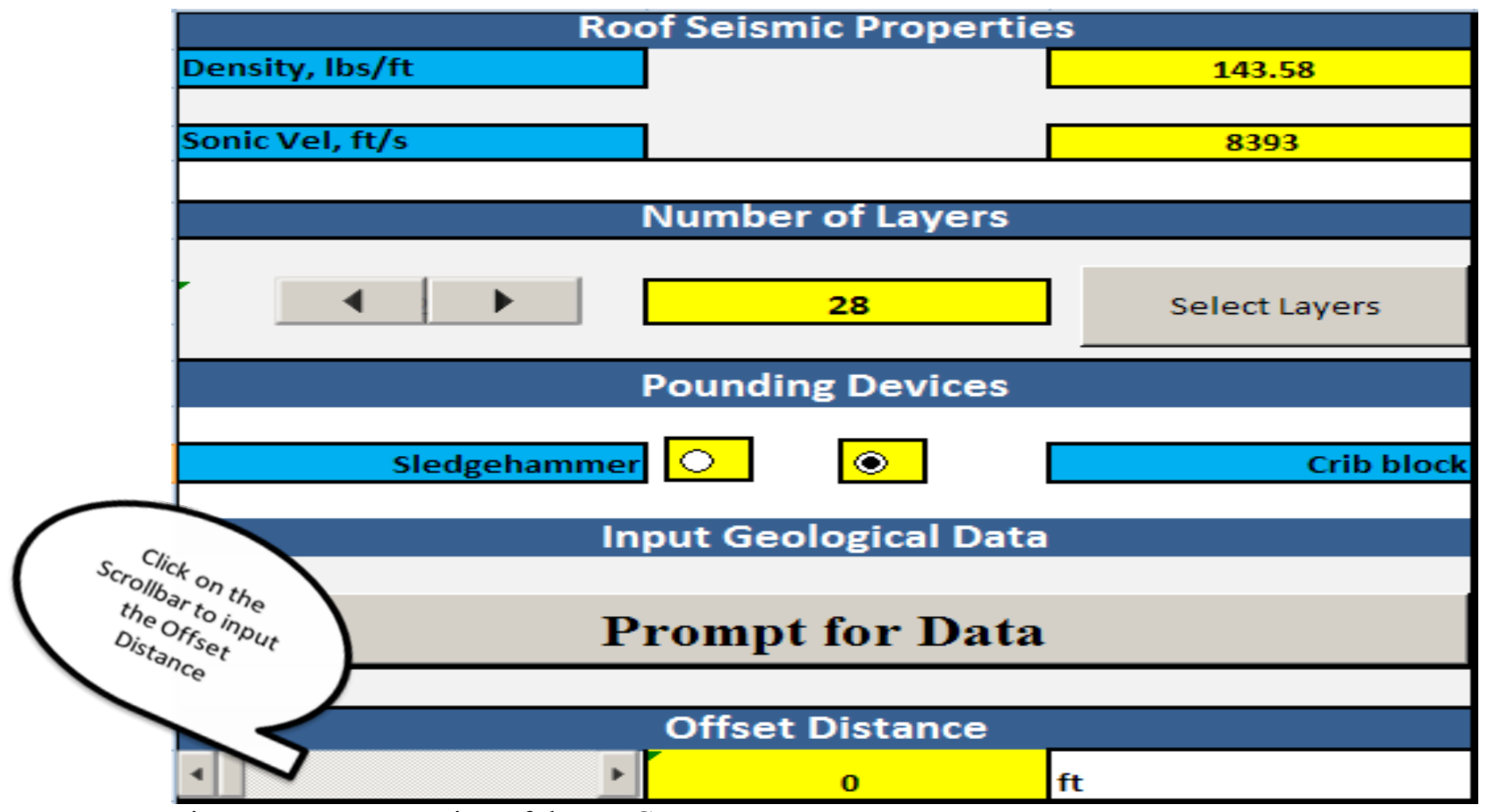

Figure 5.7. Input section of the EXCEL program. 


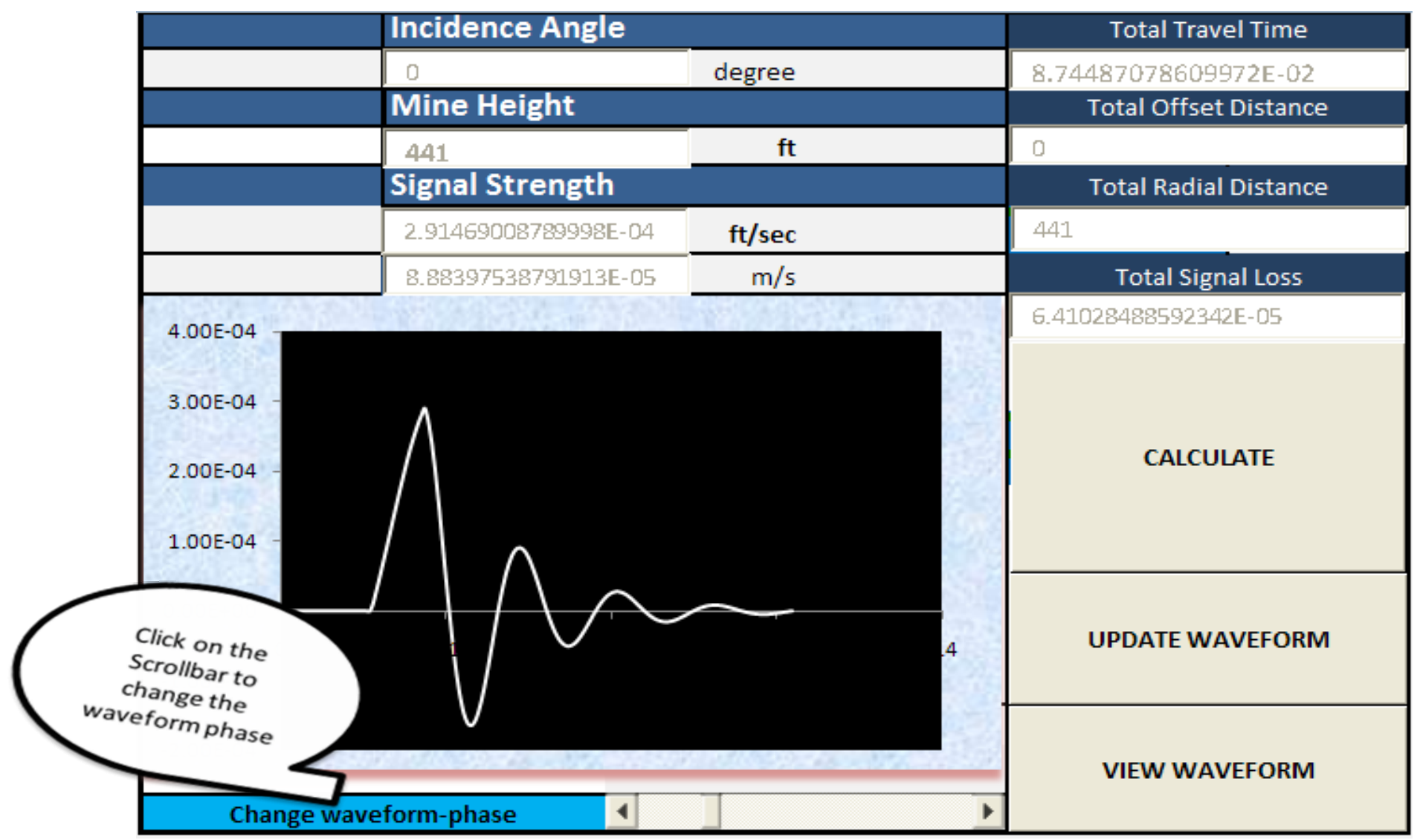

Figure 5.8. Signal waveform of the expected surface peak particle velocity.

\subsection{Validation of the PPV EXCEL Program}

In order to test the accuracy of using the PPV EXCEL program, a case study was performed using the observed data from one of our experimental fields. The site location of this field is at the Federal \#2 Mine located on Miracle Run Road, Fairview, West Virginia (see Figure 4.8-4.9). The overburden thickness is $779 \mathrm{ft}$ (see Figure 4.11) and offset distance were at $0 \mathrm{ft}, 70 \mathrm{ft}, 140 \mathrm{ft}$. Using the EXCEL program, the following parameter were entered: mine height, seismic velocity of each layer, crib block for signaling device and geophone offset distances (see Figure 5.9) 


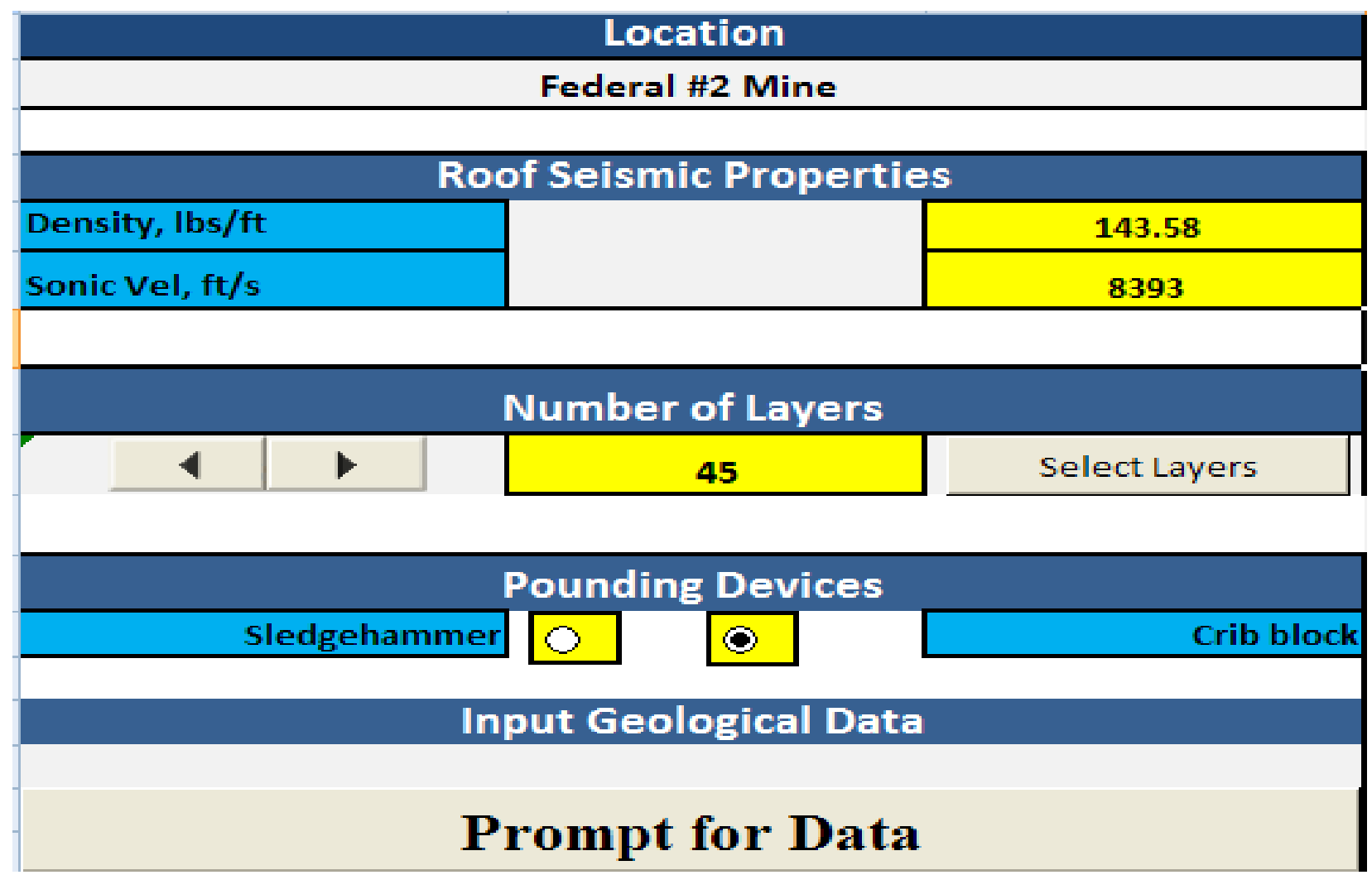

Figure 5.9. Part of input section of the EXCEL program Federal \#2 mine.

After entering all the parameters the program then solved for particle velocity at the surface as shown in Figure 5.10 which shows the expected particle velocity and the signal waveform. According to the result of the program, the expected particle velocity at zero offset distance from the pounding miner is 8.04889e-5. This signal appears to stand the chance of being overwhelmed by the surrounding noise due to its relatively low signal value when compare to the noises from the surrounding activities such as drilling, moving vehicles and so on. For this reason, the signal detection might only be achieved if the surroundings noises could be controlled such that it is made lower than the calculated signal value of $8.04889 \mathrm{e}-5$.

Comparing the result of the EXCEL program with the observation in the field, we observed that the PPV EXCEL program result is consistent with observations in the field. In the field it was concluded that no signal was detected because of the surrounding noise from the bulldozer working on the coal stockpile and also the coal cars which were moving not too far away from the 
underground signal source. This comparison between the result by the EXCEL program and the field observation provides us with the assurance that the PPV EXCEL program results are fairly reliable and might be very useful to facilitate the optimization of the future mine rescue seismic deployment plan.

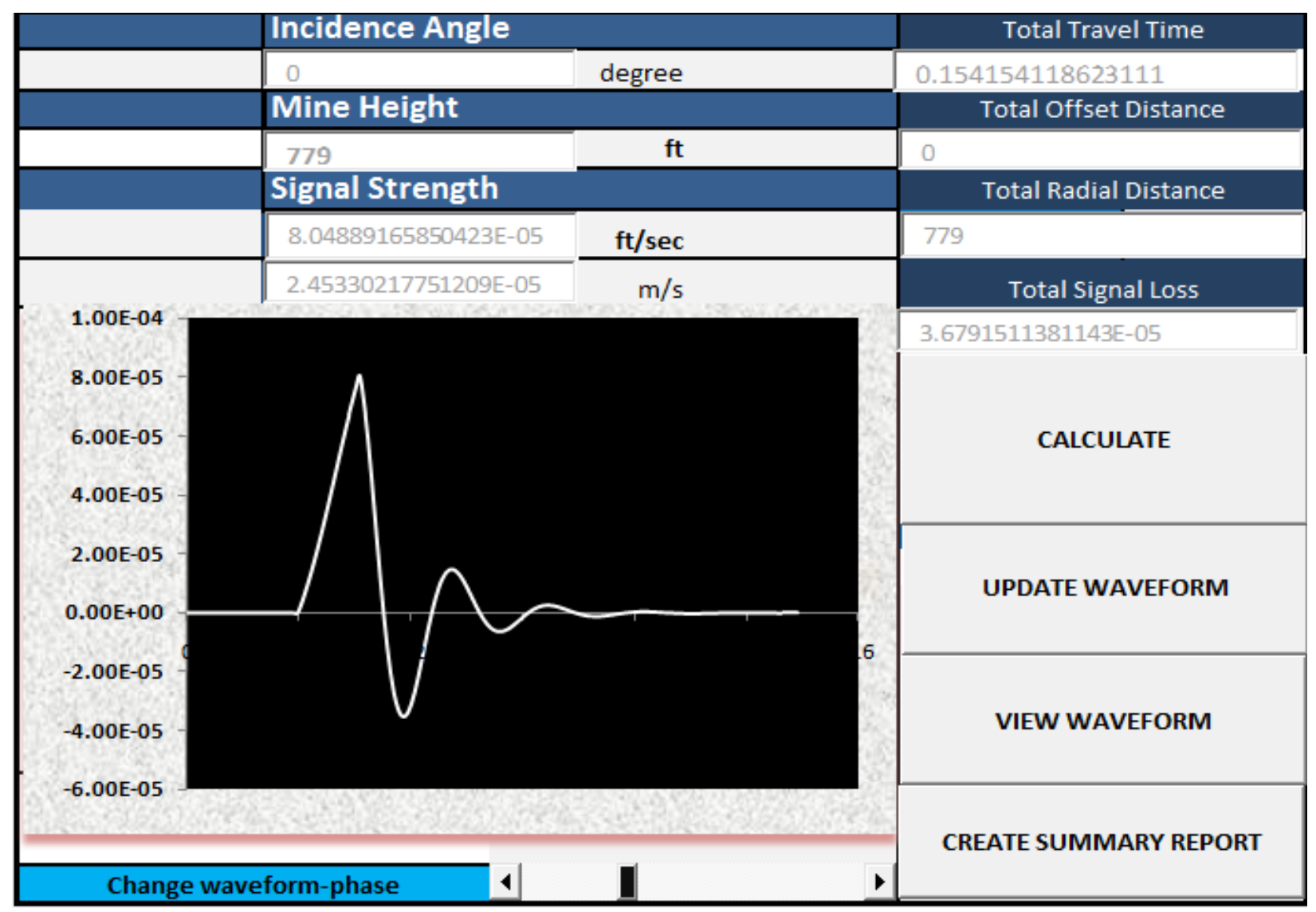

Figure 5.10. Signal Waveform of the surface peak particle velocity at Federal \#2 mine. 


\section{CHAPTER 6}

\section{CONCLUSIONS AND RECOMMENDATIONS}

An EXCEL program has been implemented in this thesis to help determine the detection capabilities of a mine rescue seismic system of a given mine. This program was developed by applying the analysis provided through a parallel study of the theoretical and experimental aspects of a mine rescue seismic system. Through this parallel study, we were able to analytically determine: the attenuation of the displacement pulse as it moves through the overburden to the surface, and experimentally determine the pounding factor which relates the pounding instruments to the frequency and amplitude of the seismic signal (Heasley, 2009). Over the course of this study two experimental tests were conducted at two different underground mines. The first test was a relative shallow (440 ft deep) mine while the second was a deeper ( $779 \mathrm{ft}$ deep) mine and in a noisy environment. Test seismic systems were installed at the mine surface with geophones being installed at various offset distances away from the source. A series of underground seismic signals with pounding devices at different locations were created. However, only the seismic signals at the 4-West Mine of Dana Mining were observed between $(0-140)$ feet away from the source. The following conclusions can be made from the analysis of the experimental tests.

- Out of the four signaling devices that were employed on different pounding locations for the test, the crib block on the rock appeared to be the strongest signal followed by the crib block on the bolt, the sledgehammer on the roof rock, and the sledgehammer on the roof bolt. Essentially, between the crib block and the sledgehammer, the crib block appeared to generate higher signal strength (at least in our study site). 
- The buried geophones provided more than twice the peak particle velocity of the surface geophones.

- The increment to the distance between the source and signal is not responsible for all of the signal attenuation. Other factors responsible for the signal loss include Inter-bed refraction/transmission at the sedimentary layers as the offset angle increase, hysteresis by the media, and geometric spreading.

- The Hilti tools did not generate very high surface particle velocities, assumedly because they generated higher frequency signals, which attenuated very fast. During the second field test, it was concluded that the background noise environment such as the mine processing plant could generate strong vibrations that could overwhelm the signal being sent from underground.

- The factors that most affect the detection capabilities of the mine rescue seismic system include: mine geology and its seismic parameters, mine depth, geophone offset distance, incidence angle, and the level of background noise.

In the theoretical analysis part of the study, a seismic attenuation model was created in EXCEL to estimate the expected signal attenuation for a given mine using the mine specific geology with average seismic parameters, horizontal offset distances of the geophones, and mine depth. The experimental pounding factor for a crib block and a sledgehammer were determined by calculating the expected attenuation of the seismic signals at the 4-West Mine of Dana Mining and then back-analyzed the peak particle velocity which were recorded during the seismic field experiment. For a crib block, pounding factor value was determined to be $4.21 \mathrm{e} 10$ and for a sledgehammer, the value is determined to be $2.29 \mathrm{e} 10$. 
By applying the analysis provided through the theoretical and field tests, the EXCEL program can calculate the expected peak particle velocity of a given mine as a function of mine depth, geology with the mine seismic parameters and geophone offset distance and the incidence angle which is determined once the offset distance is known. While trying to validate the results of the EXCEL program, we discovered a slight difference between the field data and theoretically determined data. The difference between the field data and theoretically determined data appears to reflect a number of different factors. In the case of this study these factors probably include geophone coupling and variation in the compressional velocity and thickness of the topsoil where the geophones were being installed.

This program would help the future mine rescuer to be better informed to decide the appropriate use of the seismic system based on the detection capabilities of the equipment. For instance, if the expected signal is determined to be much greater than the background noise, then the rescue team can be fairly confident that if the miners are pounding then they will detect the signal. Or, if the expected signal strength is close to, or less than, the observed background noise level, then the rescue team will need to implement appropriate procedures to reduce the background noise to detect any undergrounding pounding. On the other hand, if the expected signal is far below the background noise, then the rescue team will know that there is not much chance of detecting the trapped miners pounding, and rescue efforts can be appropriately redirected

While this study contributes to the optimization of the future mine rescue seismic plan, it is believed that there is still more areas that needs to be improved. For instance, the geophones used in the project were only able to be installed at the surface of the mine because of financial barriers. If boreholes could be drilled through the overburden from the surface and geophones 
properly installed at different depths, it is believed that a better observation of how signal attenuates through the various layers in the medium could have been made. This would allow for a better estimate of the signal attenuation of seismic energy assessments to be made at the pounding source. The two field tests which were employed to derive the pounding factor values appear to be not nearly enough. It was planned to acquire an in-house seismic system for performing detailed field tests and analysis, however this was not possible in the time allotted. It is believed that more seismic fields test at different location of the state would provide better estimated values. It is suggested that further seismic field studies for most mines in West Virginia should be studied to investigate the seismic magnitudes, frequencies, and attenuation rates of various signaling methods in varying WV geologies. To increase the chance of detecting underground signals, a "lower frequency source" will be better. It is suggested that more experimental efforts be devoted to the development of this type of signal source and a coupler to enhance the conversion of mechanical energy into seismic energy. 


\section{REFERENCES}

Abdalla, J., and Abdelwahab, A. (2006). Site Response and Earthquake Design Spectra for Central Khartoum. Bull Earthquake Engineering, 4, pp. 277-293.

Aoief, T., and Christopher, J.B. (2000). Numerical Simulation of Seismic Waves Using a Discrete Particle Scheme. Geophysics Journal International, 141, pp. 595-604.

Arthur, D.L. (1974). Earth Models: Part 8 in Survey of Electromagnetic and Seismic Noise Related to Mine Rescue Communications. BuMines Open File Report, 38, (2), pp. 8.1- 8.9.

Boatwright, J., and Fletcher, J.B. (1984). Partition of Radiated Energy Between P and S waves. Bulletin of the Seismology Society of America, 74, (2), pp. 361-376.

Christopher, T. S., and Waymond, R.S. (2000). A Finite Difference Model to Study the Elastic-Wave Interactions with Buried Land Mines. IEEE Transaction on Geosciences and Remote Sensing, 38, (4), pp. 1505-1512.

Collins, F., and Lee, C.C. (1956). Seismic Wave Attenuation Characteristics from Pulse Experiments. Geophysics, 21, (1), pp. 16-40.

David, M.B., and Boatwright, J. (1984). Average Body-Wave Radiation Coefficients. Bulletin of the Seismology Society of America, 74, (5), pp. 1615-1621.

Daniel, J. (2006). Analysis and Application of Coal Seam Seismic Waves for Detection of Abandoned Mines . M.S. Thesis. Virginia Polytechnic Institute and State University, Blacksburg, VA, pp. 45.

Durkin, B., and Greenfield, R.J. (1981). Evaluation of the Seismic System for Locating Trapped Miners. U.S. Bureau of Mines Report of Investigation, Pittsburgh, PA, RI 8567, pp. 55.

Freeman, G., and Backus, G.E. (1966). Propagation Matrices in Elastic Wave and Vibration Problems. Geophysics, 31, (2), pp. 326-332.

Futterman, W.I. (1962). Dispersive Body Waves. Journal of Geophysical Research, 67, pp. 5279-5291.

Geldart, R.E., Sheriff, E., William, M.T., and Telford, L.P. (2005). Applied Geophysics. Book, (4th ed.), pp. 770.

Greenfield, R. (1978). Seismic Radiation from a Point Source on the Surface of a Cylindrical Cavity. Geophysics, 43, (6), pp. 1071-1082.

Greenfield, R. (1981). Detection Range and Arrival Time Estimates, Part 2 in Survey of Electromagnetic and Seismic Noise Related to Mine Rescue Communications. Seismic Detection 
and Location of Isolated Miners (USBM Contract HO 122026). BuMines Open File Report, 38, (2), pp. 2.1-2.30.

Heasley, K.A. (2006). Seismic Field Test at 4 West Mine. Project Report, Department of Mining Engineering, West Virginia University, pp. 10.

Heasley, K.A., Peng, S., and Luo, Y. (2009). Developing and Field Testing of a Seismic Location System for Trapped Coal Miners. Progress Report, Department of Mining Engineering, West Virginia University, Morgantown, pp. 13.

Heasley, K.A. (2009). Verbal discussion.

Hannon, W.J. (1964). An Application of the Haskell-Thomson Matrix Method to the Synthesis of the Surface Motion Due to Dilatational Waves. Bulletin of the Seismology Society of America, 54, (6), pp. 2067-2079.

Hans, B., and Carcione, J.M. (2003). Velocity and Attenuation in Partially Saturated Rocks: Poroelastic Numerical Experiments. Geophysical Prospecting, 51, pp. 551-566.

Hilti Corporation Products Webpage. Website: www.hilti.com.

Hiro, K. (1967). Spectrum of P and PcP Relation to the Mantle-Core Boundary and Attenuation in the Mantle. Journal of Geophysical Research, 72, (2), pp. 559-571.

Howell, B.F., and Kaukonen, E.K. (1951). Attenuation of Seismic Waves Near an Explosion. Internal Constitution of the Earth, pp. 382-407.

Institute of Physics Webpage. Website: www.physicsworld.com.

Kennet, B.L. (1983). Seismic Wave Propagation in Stratified Media. Cambridge University Press, New York, pp. 298.

Knopoff, L., and MacDonald, J.F. (1962). Attenuation of Small Amplitude Stress Waves in Solids. Reviews of Modern Physics, 30, (4), pp. 1178-1192.

Krohn, C. (1984). Geophone Ground Coupling. Geophysics, 49, (6), pp. 722-731.

Kuo, J.T. (1974). Theoretical Seismic Signal Source and Transmission Characteristics. Part 7 in Survey of Electromagnetic and Seismic Noise Related to Mine Rescue Communications. Seismic Detection and Location of Isolated Miners (USBM Contract HO 122026). BuMines Open File Report, 38, (2), pp. 7.1-7.33.

Leblanc, S.J. (1967). Truncated Crustal Transfer Functions and Fine Crustal Structure Determination. Bulletin of the Seismology Society of America, 57, (4), pp. 719-733. 
Mallick, S. (1984). A Simple Approximation to the P-wave Reflection Coefficient and its Implication in the Inversion of Amplitude Variation with Offset Data. Geophysics, 49, (10), pp. 1637-1648.

MSHA. (1998). Coal Fatalities from 1900 through 2001. Website: www.msha.gov.

Nafe, J. (1962). Reflection and Transmission Coefficients at a Solid-Solid Interface of High Velocity Contrast. Bulletin of the Seismological Society of America, 52, (4), pp. 923-955.

National Academy of Engineering. (1970). Committee on Mine Rescue and Survival Techniques, Mine Rescue and Survival. BuMines OFR 4-70, pp. 88.

National Geoscience Database of Iran Webpage. Website: http://www.ngdir.ir.

Powell, J.A., and Watson, J.A. (1976). Seismic Detection of Trapped Miners Using In-Mine Geophones. U.S. Bureau of Mines Report of Investigation, Pittsburgh, PA, RI 8158, pp.12.

Radar Technology Tutorial Webpage. Website: www.radartutorial.eu.

Sarker, G., and Geoffrey, A. (1998). Comparison of Seismic Body Wave and Coda Wave Measure. Pure and Applied Geophysics, 153, pp. 665-683.

Sung, T.Y. (1953). Vibrations in Semi-Infinite Solids Due to Periodic Surface Loading in Symposium on Dynamic Testing of Soils. American Society for Testing and Materials, Philadelphia, PA., pp. 35-63.

Swanson, P.L., Estey, L.H., and Billington, S. (1992). Accuracy and Precision of Microseismic Events Locations in Rock Burst Research Studies. U.S. Bureau of Mines Report of Investigation, Pittsburgh, PA, RI 9395, pp. 40.

Tooley, R.D., and Spencer, T.W. (1965). Reflection and Transmission of Plane Compressional Waves. Geophysics, 30, (4), pp. 552- 570.

Wang, T., and Rokhlin, S.I. (2001). Stable Reformation of Transfer Matrix Method for Wave Propagation in Layered Anisotropic Media. Ultrasonic, 39, pp. 413-424.

Weir-Jones Group Products Webpage. Website: www.weir-jones.com.

West Virginia Mine Safety Roundtable on Seismic Miner Location. (2006). Working Draft on Seismic Miner Location, pp. 12.

West Virginia Mine Safety Technology Task Force. (2006). Report and Mine Safety Recommendation to the Director of the Office of Miners' Health, Safety and Training on Sago Mine Disaster, pp. 137. 
Westinghouse Electric Corporation Coal Mine Rescue and Survival. (1971). Communications Location Subsystem (USBM Contract HO 101 262). BuMines Open File Report, 9, (2), pp. 258.

White, J. E. (1965). Seismic Waves: Radiation, Transmission, and Attenuation. McGraw-Hill Book Co., Inc., New York, pp. 302.

Wolf, A. (1941). The Equation of Motion of a Geophone on the Surface of an Elastic Earth. Society of Exploration Geophysics, 6, pp. 115-116.

Young, G., and Braille, L.W. (1976). A Computer Program for the Application of Zoeprittzs Amplitude Equations and Knott's Energy Equations. Bulletin of the Seismological Society of America, 66 (6), pp. 1881-1885.

Zheng, Y., and Tsutomu, U. (2003). Seismic Response Predictions of Multi-Span Steel Bridges through Pushover Analysis. Earthquake Engineering and Structural Dynamics, 32 (8), pp. 1881-1885.

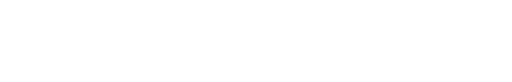

